University of Louisville ThinkIR: The University of Louisville's Institutional Repository

Electronic Theses and Dissertations

5-2019

\title{
Innovation and equity in public health research : testing arts-based methods for trauma-informed, culturally-responsive inquiry.
}

Tasha Golden

University of Louisville

Follow this and additional works at: https://ir.library.louisville.edu/etd

Part of the Community Health and Preventive Medicine Commons, Domestic and Intimate Partner Violence Commons, Health Communication Commons, Health Services Research Commons, Other Mental and Social Health Commons, Public Health Education and Promotion Commons, and the Women's Health Commons

\section{Recommended Citation}

Golden, Tasha, "Innovation and equity in public health research : testing arts-based methods for trauma-informed, culturallyresponsive inquiry." (2019). Electronic Theses and Dissertations. Paper 3233.

https://doi.org/10.18297/etd/3233

This Doctoral Dissertation is brought to you for free and open access by ThinkIR: The University of Louisville's Institutional Repository. It has been accepted for inclusion in Electronic Theses and Dissertations by an authorized administrator of ThinkIR: The University of Louisville's Institutional Repository. This title appears here courtesy of the author, who has retained all other copyrights. For more information, please contact thinkir@louisville.edu. 
INNOVATION AND EQUITY IN PUBLIC HEALTH RESEARCH:

TESTING ARTS-BASED METHODS FOR TRAUMA-INFORMED, CULTURALLY-

RESPONSIVE INQUIRY

\author{
By \\ Tasha Golden \\ M.A., Miami University, 2012
}

\begin{abstract}
A Dissertation
Submitted to the Faculty of the

School of Public Health and Information Sciences at the

University of Louisville

in Partial Fulfillment of the Requirements

for the Degree of

Doctor of Philosophy

in Public Health Sciences

Department of Health Promotion and Behavioral Sciences

University of Louisville

Louisville, Kentucky
\end{abstract}

May 2019 
Copyright 2019 by Tasha Lee Golden

All rights reserved 

INNOVATION AND EQUITY IN PUBLIC HEALTH RESEARCH:

TESTING ARTS-BASED METHODS FOR TRAUMA-INFORMED, CULTURALLY-

RESPONSIVE INQUIRY

\author{
By \\ Tasha Golden \\ M.A., Miami University, 2012 \\ A Dissertation Approved on
}

January 25, 2019

by the following Dissertation Committee:

$\overline{\text { Monica L. Wendel, Dr.P.H., M.A., M.P.H. }}$

Dissertation Chair

Brandy Kelly Pryor, Ph.D.

Kathryn Hopkins, Ph.D., HSPP

Mary P. Sheridan, Ph.D. 


\section{DEDICATION}

This dissertation is dedicated to the many young women

whose stories have taught and shaped me,

and to Justin Golden, my partner in all things,

who models compassion, responsiveness, and genuine inquiry. 


\section{ACKNOWLEDGMENTS}

My ongoing thanks to Dr. Monica Wendel, my dissertation director and friend, whose attentive presence from the outset allowed her to see my work and its trajectory, and to welcome it into new spaces. Her commitments to equity, authenticity, and creativity have been vital guides. "Do the next thing."

I am also deeply grateful to my committee members: Dr. Mary P. Sheridan, Dr. Katy Hopkins, and Dr. Brandy Kelly Pryor. This interdisciplinary committee was a force of expertise, sensitivity, awareness, and rich insight. What a gift to have journeyed with these scholars.

My thanks also to Kristen Miller and Sarabande Books, whose partnership in Louisville has been crucial to this effort, and to Dr. Mullins, Erika Day, and the Louisville Metro Youth Detention Center for recognizing the value of my research and programming. And such gratitude to Dr. Susan Buchino and Dr. Aishia Brown, who helped build foundations for Project Uncaged in Louisville; their friendship is one of the great gifts of this work.

I am deeply grateful to Beth Dixon and to Project Jericho in Springfield Ohio, whose invitation years ago to participate in their work set me on a path to researching the intersections of communication, well-being, equity, and the arts. And immense thanks always to my family, who have followed this work in its many forms with love and interest, and to my partner, whose laughter, presence, and profound listening have been immeasurable supports.

And finally, my deepest gratitude to the brave, insightful women whose writing I have been honored to read, consider, recount and, ultimately, invite into another discourse. What an incalculable privilege it has been to bear witness to this art, this truth, this knowledge, these stories. 


\begin{abstract}
INNOVATION AND EQUITY IN PUBLIC HEALTH RESEARCH:

TESTING ARTS-BASED METHODS FOR TRAUMA-INFORMED, CULTURALLY-

RESPONSIVE INQUIRY

Tasha Golden
\end{abstract}

January 25, 2019

As the field of Public Health has shifted from a biomedical paradigm to a social ecological approach, focusing on health equity and social determinants of health, it has increasingly acknowledged the roles of culture and trauma in health outcomes. The field has responded by modifying some research practices, but continues to prioritize conventional methodologies - with few options designed to foreground trauma- and cultural-responsiveness. Unfortunately, to the extent that conventional approaches require health to be defined, communicated, or valued according to dominant norms, they risk perpetuating inequities. Health equity therefore requires the development of research methodologies that increase inclusivity and access, elicit and apply diverse knowledges, reduce intrusion, and incorporate sociocultural factors.

Using design thinking, and drawing upon critical theory, hermeneutics, photovoice, and arts in health research, this study developed and tested three methodological prototypes for researching a potentially traumatizing topic (violence) among a vulnerable population (young women ages 11-21). The first prototype collected 
creative writing from an ongoing youth detention intervention; the second utilized a themed citywide writing contest; and the third obtained the script for a play about sexual and relational violence, written and performed by local survivors without researcher elicitation.

Methods:

All prototype-generated data (combined $n=126$ ) were iteratively analyzed for documented experiences, needs, assets, and priorities related to violence. Four conventional violence-related surveys, administered among the three sample populations (combined $n=1862$ ), were also analyzed. Extensive comparative evaluations were then conducted of all findings and methods, assessing quality/quantity of information, trauma responsiveness, and cultural responsiveness.

Results:

Findings from the arts-based methods addressed 64 to 89 percent of the same variables as the surveys, while the surveys addressed only 36 to 49 percent of the artsbased data variables. The three prototypes juxtaposed needs with assets, accommodated expressive choice, and involved minimal researcher intrusion-rendering them trauma and culturally responsive. They also generated impactful insights inaccessible via surveys, such as unconventional definitions of violence. The prototypes aided disclosure (thus improving data), were time- and cost-effective, and offered significant potential for research reciprocity. As such, they offer promising models for continued methodological innovation in advancement of health equity. 


\section{TABLE OF CONTENTS}

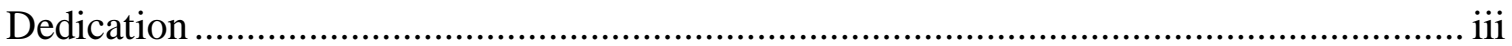

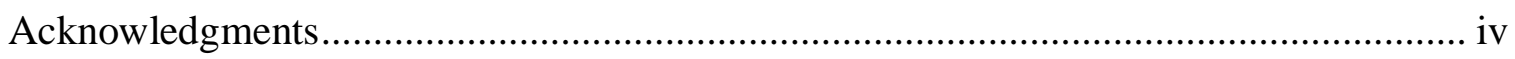

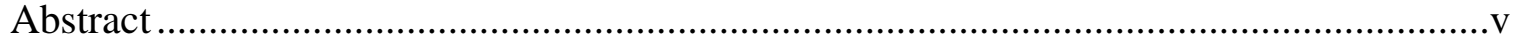

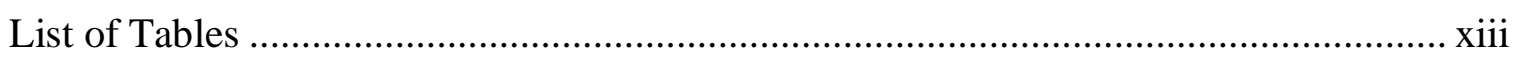

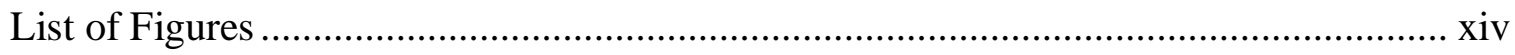

Chapter One: Introduction: Health Equity and the Need for Innovation .............................1

1.1: Cultural Responsiveness ........................................................................

1.2: Trauma Responsiveness ...........................................................................

1.3: Linking Cultural and Trauma Responsiveness ................................................5

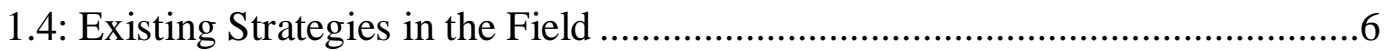

1.4.1 Minor Adaptations .......................................................................

1.4.2 Participatory Research ....................................................................

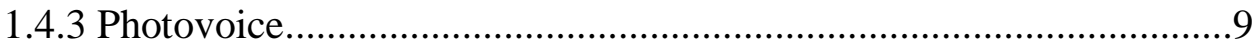

1.5: Opportunities to Make Advances ............................................................10

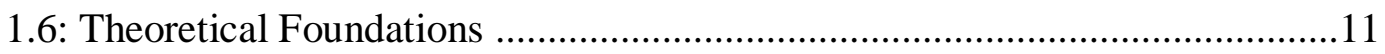

1.6.1 Critical Theory and Intersectionality ...............................................11

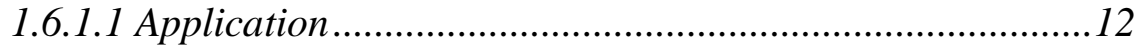

1.6.2 Expressive Writing and Cognitive Change Theory ...........................13

1.6.2.1 Application .......................................................................

1.6.3 Arts in Health Research ..................................................................15

1.6.4 Hermeneutic Theory ......................................................................18

1.6.4.1 Application ……………………………......................18

1.6.5 Design Thinking and SystemsTheory ……………….......................19

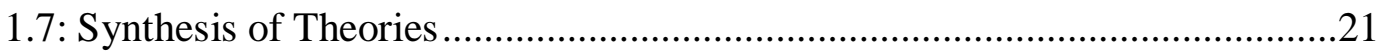


1.8: Authorial Positionality

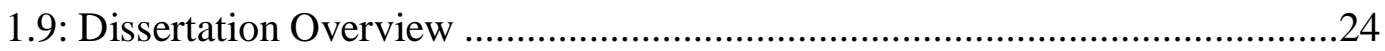

Chapter Two: Literature Review: Foundations for Innovation.........................................26

2.1: Conventional Methodologies: Hegemony and Inequity ..................................27

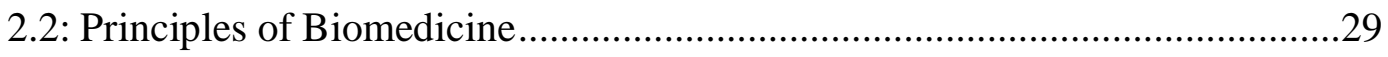

2.3: Critiques of Conventional Approaches ..........................................................

2.4: Photovoice as a Foundation for Methodological Innovation .............................34

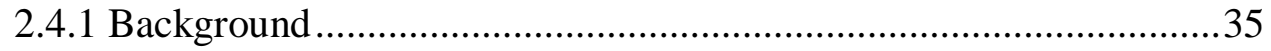

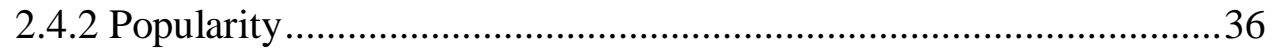

2.4.3 Four Limitations ..............................................................................

2.4.3.1 Community Centered? ............................................................

2.4.3.2 Ownership and Copyright Concerns ...................................40

2.4.3.3 Problematic Empowerment Rhetoric ....................................41

2.4.3.4 Broken Promises Regarding Policy Influence ....................42

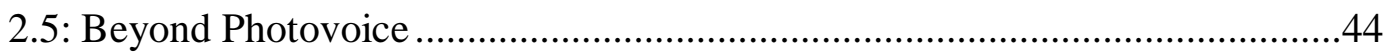

2.5.1 Photovoice as an Arts-Based Method ................................................4

2.5.1.1 "Art" in the Literature? .......................................................44

2.5.1.2 Community Art Is Data .....................................................46

2.5.1.3 Structural Constraints (Again) ............................................4

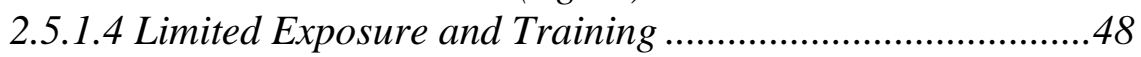

2.6: Untapped Potential, Implications, and Exploration .......................................49

2.6.1 Aiding Disclosure, Improving Data ..................................................49

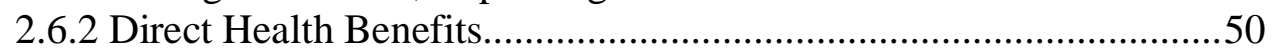

2.6.3 Framing and Storytelling ………………………........................52

2.7: Next Steps: Building on Photovoice and Theoretical Frameworks .................53

2.7.1 Trauma-informed practice requires expressive options ......................53

2.7.2 Move beyond photography ................................................................54

2.7.3 Cultural responsiveness requires exploration .....................................55

2.7.4 Look for the art already being made ..................................................55

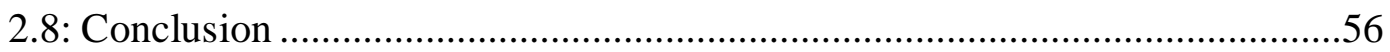

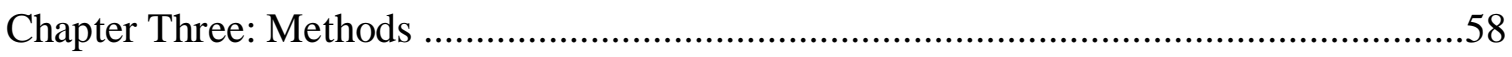

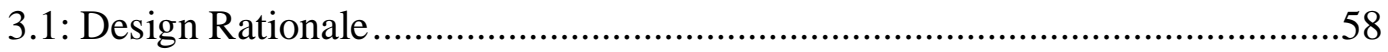




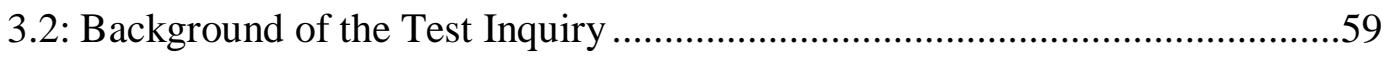

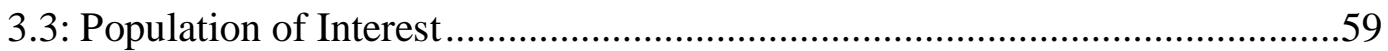

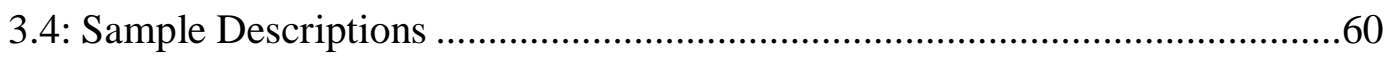

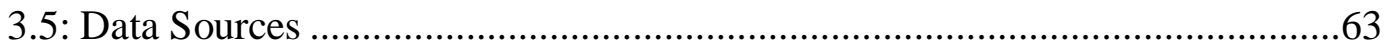

3.5.1 Qualitative Data Sources...........................................................63

3.5.2 Quantitative Data Sources..........................................................6 65

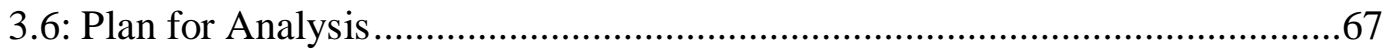

3.6.1 Arts-Based Qualitative Inquiry ...............................................67

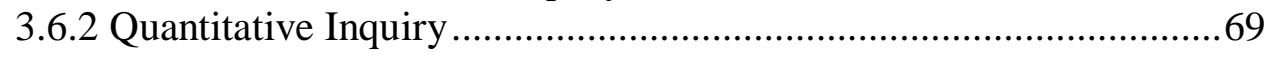

3.7: Population Comparisons ................................................................. 71

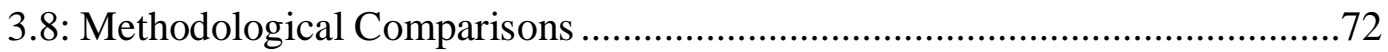

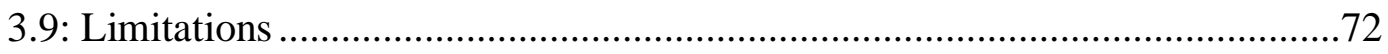

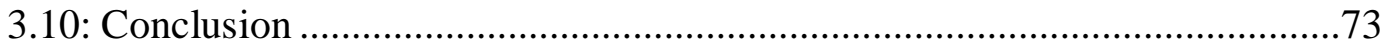

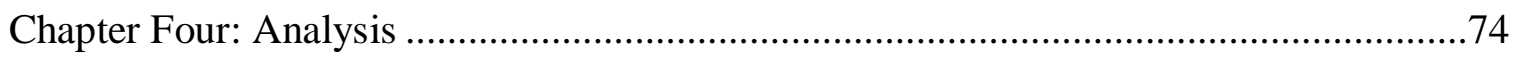

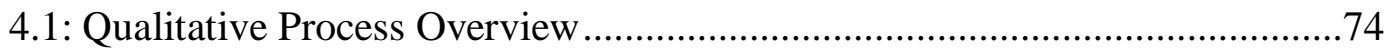

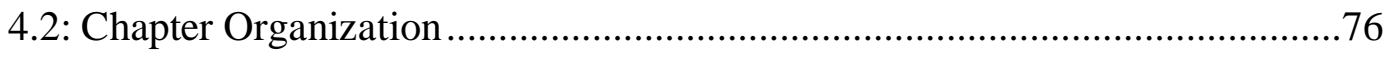

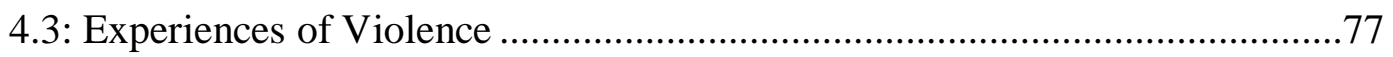

4.3.1 Experiences of Violence - Interpersonal........................................78

4.3.1.1 Domestic/Intimate Partner Violence (D/IPV)..................78

4.3.1.2 Trauma ............................................................................ 79

4.3.1.3 Fear/Threat ...............................................................8 80

4.3.1.4 Overview: Remaining Interpersonal Violence Codes........81

4.3.1.5 Additional Findings and Population Disparities...............83

4.3.2 Experiences of Violence - Structural......................................... 85

4.4: Needs and Priorities Associated with Violence ...........................................8

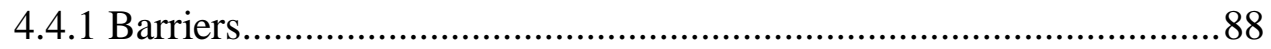

4.4.1.1 Mental Health ............................................................... 88

4.4.1.2 Gender Roles/Expectations ...........................................89

4.4.1.3 No One to Depend On....................................................90

4.4.1.4 No One Notices .................................................................90

4.4.1.5 No One Listens/Hears ....................................................91

4.4.1.6 Deceptive Love ...........................................................91

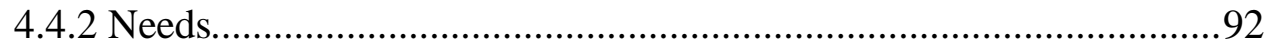


4.4.2.1 To ACT; Take Responsibility .........................................93

4.4.2.2 Speaking Out/Voice .......................................................94

4.4.2.3 Self-Worth .......................................................................95

4.4.2.4 Social Supports .................................................................95

4.4.2.5 Safety ........................................................................96

4.4.2.6 Overview: Remaining Needs .......................................96

4.4.3 Effects ..........................................................................................99

4.4.3.1 Guilt/Shame/Regret/Self-Blame ......................................98

4.4.3.2 Hopelessness/Overwhelm ..............................................99

4.4.3.3 Lingering Effects .............................................................99

4.4.3.4 Bruises/Scars................................................................99

4.4.3.5 Anger/Rage .................................................................. 100

4.4.3.6 Overview: Remaining Effects......................................100

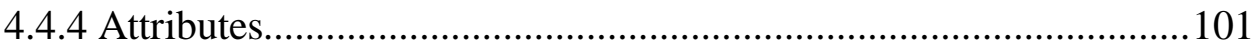

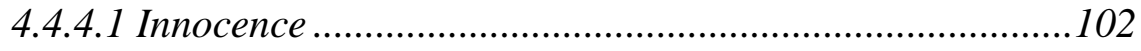

4.4.4.2 Authenticity/Inner Self ................................................102

4.4.4.3 Identity Claiming/Defining ............................................ 103

4.4.4.4 Overview: Remaining Attributes...................................103

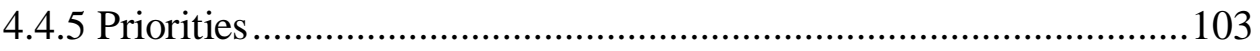

4.5: Assets In Building A Safer/Healthier City ...............................................104

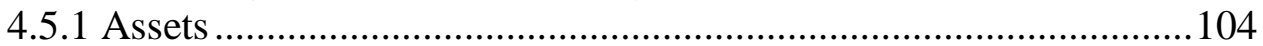

4.5.1.1 Confidence, Empowerment ..........................................105

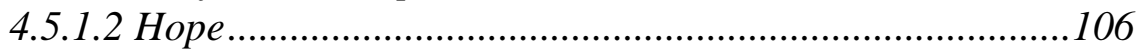

4.5.1.3 Encouraging/Advising/Advocating for Others.................107

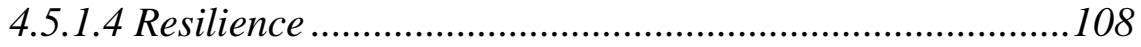

4.5.1.5 Perseverance/Persistence .............................................108

4.5.1.6 Overview: Remaining Assets.......................................109

4.6: Values Related To Violence ..................................................................110

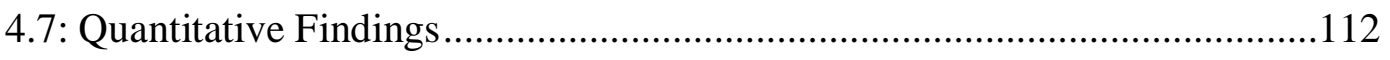

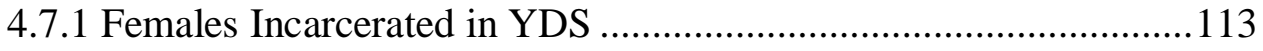

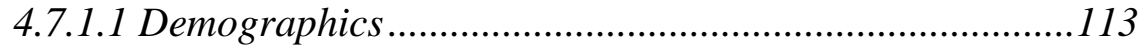

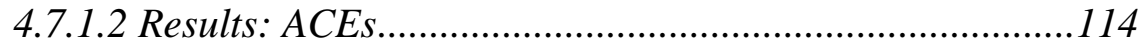

4.7.1.3 Results: Trauma Symptoms............................................116

4.7.1.4 Results: Resilience .....................................................117

4.7.2 Females Ages 11 to 21 in Greater Louisville................................119

4.7.2.1 Demographics .......................................................... 120

4.7.2.2 Results: Exposure to Violence ........................................120

4.7.2.3 Results: Community Involvement, Civic Engagement .....122

4.7.2.4 Results: Attitudes toward Violence ...................................124

4.7.2.5 Results: Social Supports ..............................................125

4.7.2.6 Results: Mann-Whitney U Tests....................................126

4.7.2.7 Results: Kruskal-Wallis H Tests ..................................126

4.7.2.8 Results: Spearman Rand Order Correlation Tests ..........127 
4.7.3 Female Students at the University of Louisville...........................128

4.7.3.1 CardSafe Survey: Introduction ......................................129

4.7.3.2 CardSafe Demographics ..............................................129

4.7.3.3 CardSafe Results: Experiences of Violence .....................130

4.7.3.4 CardSafe Results: Witnessing Violence ..........................131

4.7.3.5 CardSafe Results: Personal Response/Action .................133

4.7.3.6 Well-Being and Resilience Survey: Introduction.............134

4.7.3.7 WBR Demographics..................................................... 135

4.7.3.8 WBR Results: Experiences of Violence and Safety ..........136

4.7.3.9 WBR Results: Needs and Barriers ...................................136

4.7.3.10 WBR Results: Assets, Attributes, and Values................141

4.8: Summary of Findings Regarding Violence ........................................... 142

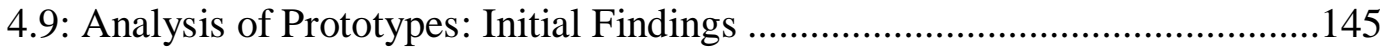

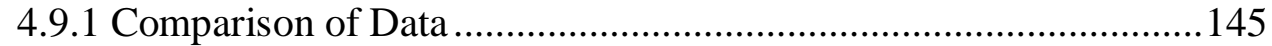

4.9.2 Survey Methods: Initial Results .............................................. 152

4.9.3 New Methods: Initial Results................................................... 154

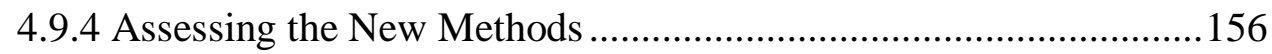

4.9.4.1 Desired Outcomes .........................................................156

4.9.4.2 Potential Benefits of Arts-Based Methods ...................... 158

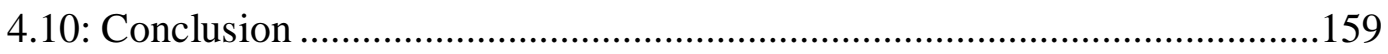

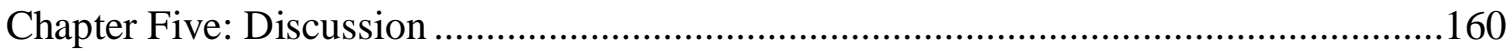

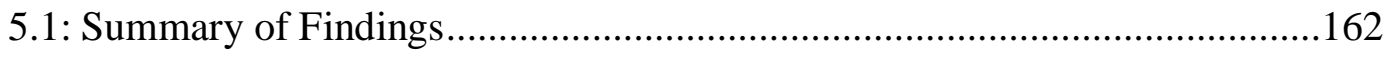

5.2: Interpretation and Contextualization of Findings ...................................... 164

5.2.1 Comparing Surveys to Prototyped Methods ................................. 164

5.2.2 The Importance of Localized, Qualitative Data...........................165

5.2.3 Moving Health Beyond the Individual ....................................... 166

5.2.4 Expanding Terms and Inclusivity .......................................... 167

5.2.5 Unique Benefits ................................................................. 168

5.2.5.1 Trauma- and Cultural-Responsiveness.........................168

5.2.5.2 Increased Interest, Participation ...................................168

5.2.5.3 Assets-Based Context ...................................................169

5.2.6 Attributes of Each Prototype.................................................... 170

5.2.6.1 YDS Intervention ...................................................... 170

5.2.6.2 Writing Contest .......................................................... 170

5.2.6.3 Pre-existing Art (Wildfire Play)....................................171

5.2.7 Methods Integration.......................................................... 172

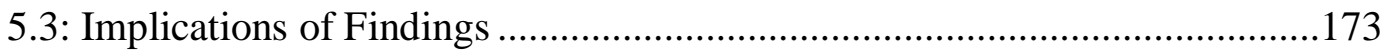

5.3.1 Implications for Research Design and Health Equity................... 173

5.3.2 Implications for Public Health Education and Training ................176 
5.3.3 Implications for Social Ecological Thinking ............................. 177

5.3.4 Implications for Needs Assessments ........................................... 179

5.3.5 Implications for Ethical Considerations...................................... 179

5.3.6 Implications for Health Communications .................................. 180

5.3.7 Implications for Interprofessional Communications ..................... 181

5.3.8 Implications for Research Funding .........................................181

5.4: Limitations and Future Directions ........................................................... 182

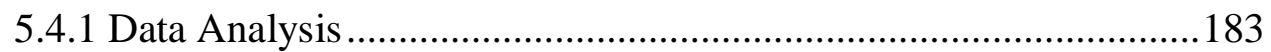

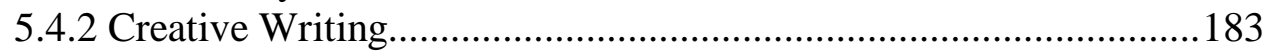

5.4.3 Direct Health Benefits................................................................ 185

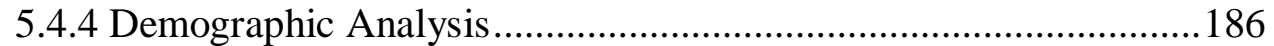

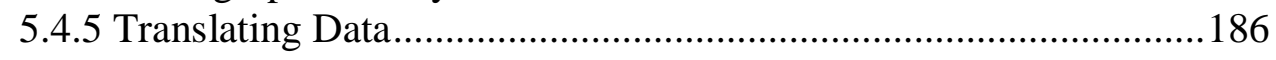

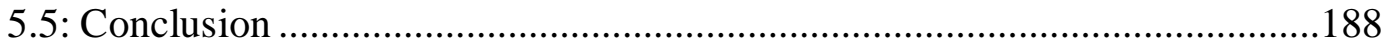

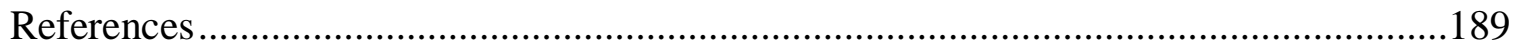

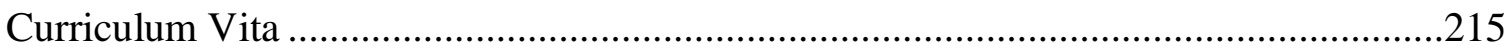




\section{LIST OF TABLES}

Table 4.1: Frequency Counts by Theme and Data Set..................................................... 77

Table 4.2: Frequency Counts, Interpersonal Violence ................................................... 83

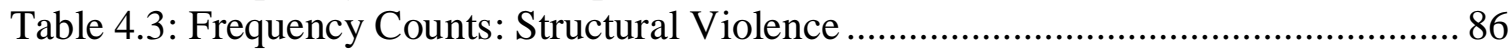

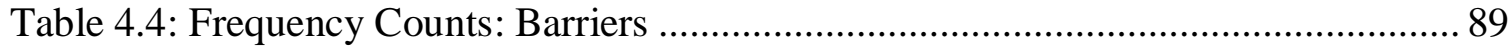

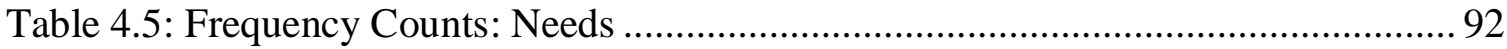

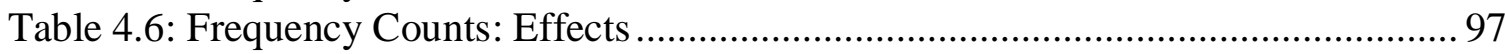

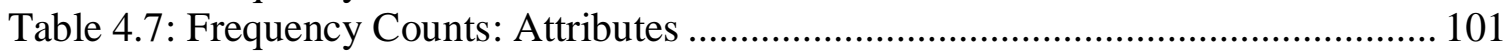

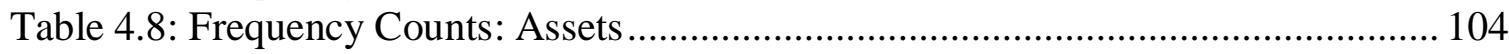

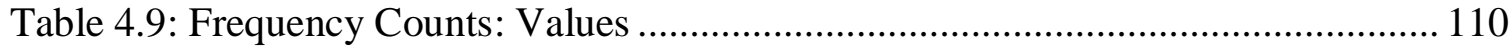

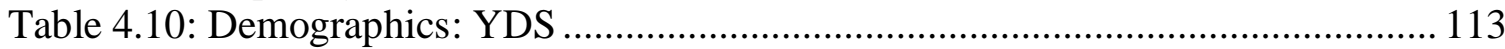

Table 4.11: Specific ACEs and their Frequencies ..................................................... 115

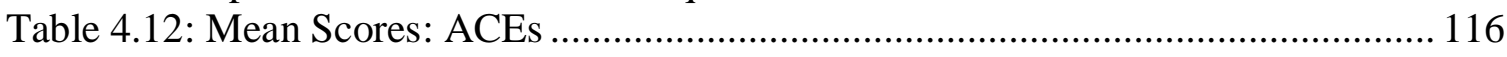

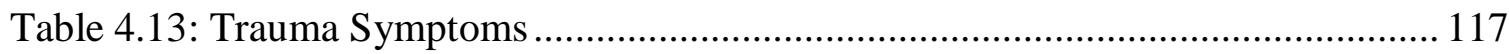

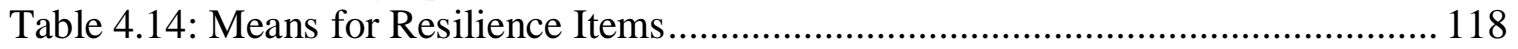

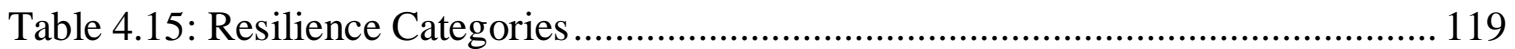

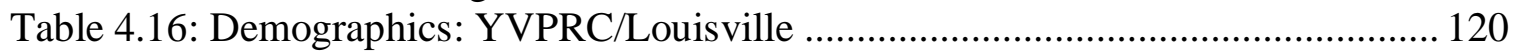

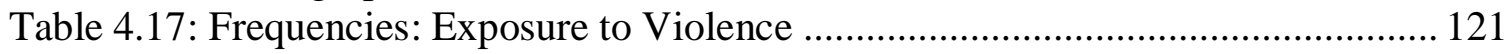

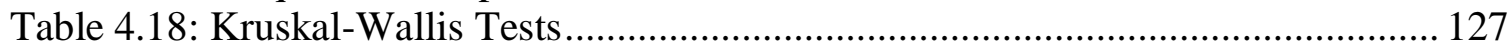

Table 4.19: Violence Exposure, Social Supports, Beliefs ........................................... 128

Table 4.20: Demographics: CardSafe Survey ............................................................. 129

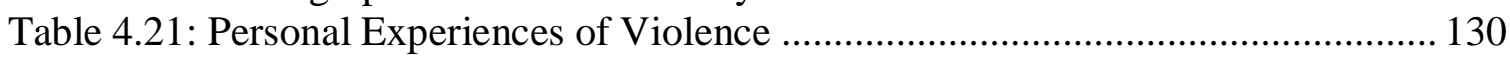

Table 4.22: Witnessed/Suspected Violence ................................................................ 132

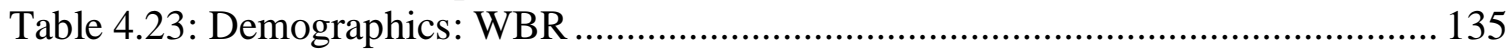

Table 4.24: Frequency with which women obtained verbal consent ........................... 137

Table 4.25: Self-Reported Health by Sexual Orientation .......................................... 139

Table 4.26: Frequency of Issues Affecting Women's Academic Status ....................... 140

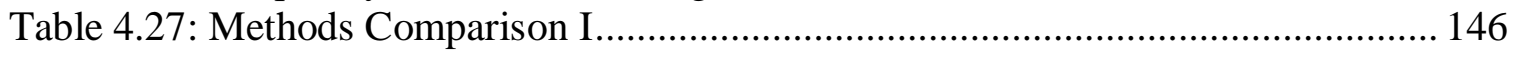

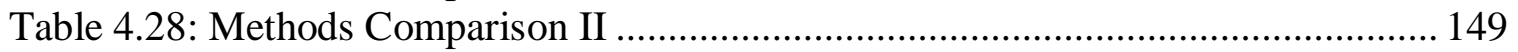

Table 4.29: Assessment of Desired Outcomes........................................................... 156 


\section{LIST OF FIGURES}

Figure 1.1: Public Health Innovation Model (Lister et al., 2017, p. 4)........................... 20

Figure 2.1: Popularity of photovoice over time .......................................................... 38

Figure 4.2: Themes grouped according to RQ1 Categories......................................... 76 


\section{CHAPTER ONE: INTRODUCTION}

"The language we have in that world is not large enough for the territory that we've already entered." - David Whyte

As the field of Public Health has shifted from a biomedical paradigm to a social ecological approach, attending to health inequities and the social determinants of health, it has increasingly acknowledged the role of both culture and trauma in influencing and determining health outcomes (Singer, Dressler, \& George, 2016; Napier et al., 2014; Trujillo \& Plough, 2015; Magruder et al., 2016). For example, Napier et al. (2014) argued that "failure to recognise [sic] the intersection of culture with other structural and societal factors creates and compounds poor health outcomes" (p. 1607), and that "a failure to acknowledge culture leaves its negative effects unaddressed and its positive potential for providing new models of thinking unrealised $[$ sic $] "$ (p. 1611). Similarly, the Substance Abuse and Mental Health Services Administration (SAMHSA) reported the widespread impact of both individual and community trauma across sectors, systems, and service agencies, indicating the need for population- and systems-level responses and pointedly arguing that "clinical interventions are not enough" (SAMHSA, 2014, p. 6). This project responds to these needs by theorizing, developing, and evaluating innovative methods of 
public health inquiry designed to improve cultural- and trauma-responsiveness while also improving data quality from marginalized populations and creating opportunities for improved advocacy.

This introduction elaborates on the need for greater cultural responsiveness and improved trauma-informed practices, then glosses current strategies used in the field to respond to these needs, noting the demand for ongoing innovation and experimentation. It then discusses the theoretical frameworks in which the current research is grounded and identifies the current study's driving research questions before providing an overview of subsequent chapters.

\subsection{Cultural Responsiveness}

An increase in discussions and considerations of culture in public health practice has followed in part from research regarding the health consequences of having marginalized or wholly dismissed knowledges, practices, values, or behaviors that lie beyond the norms of the dominant culture and its narratives (Stanton, 2014; Trickett et al., 2011). ${ }^{1}$ Countering the potentially oppressive nature of traditional or uncritical public health practices requires intentional, iterative inquiry regarding a given community's dynamic definitions, beliefs, and values related to health, well-being, and agency

\footnotetext{
${ }^{1}$ Throughout the 1990s, as such marginalization was shown to detrimentally affect health care provision and patient adherence, "cultural sensitivity"-recognition that differences exist between cultures - was incorporated into health care education, administration, and training (Brownlee \& Lee, 2018). This concept was eventually sidelined in favor of cultural competency (SAMHSA, 2016), which has itself been replaced in some contexts by cultural humility (Peters, 2016) - given that true "competency" can never quite be attained (see Beavis et al., 2015, p. 6). This author prefers the term cultural responsiveness (DicksonHughes et al., 2009; Gill \& Babacan, 2012) as it implies not only a stance or knowledge base but responsive (critical, informed) action. It is also worth noting Django Paris's (2012) term cultural sustainment, emerging from the field of education. Paris argues that public initiatives should not only respond to culture but also sustain and nourish it.
} 
(Bhattacharya, 2009; Stanton, 2014; Brown \& Strega, 2005; Beavis et al., 2015; Dahm, 2014).

Growing considerations of culture have also followed from a recognition of "the recent changing demographics in the U.S.... with the shifting diversity and proportion of populations of different social classes, cultural backgrounds, social structures, and economic restraints" (Singer, Dressler, \& George, 2016, p. 238). These factors inevitably influence health communications, health care delivery and access, provider-consumer relationships, intervention effectiveness, data accuracy, and approaches to community capacity-building. As Knight (2014) argued, "research must reflect community priorities, be meaningful to the community, and be better connected to the lived experiences of people most affected by health inequities. In this way, different kinds of knowledge inherent in communities should be valued" (p. 193).

\subsection{Trauma Responsiveness}

Meanwhile, the expansion of research in recent decades regarding trauma definitions, prevalence, impacts, and responses in health fields writ large illuminates the "staggering" impact of trauma "for both communities and individuals" (Magruder, Maclauglin \& Borbon, 2017, p. 2), leading to increased calls for a public health response (Magruder et al., 2016; SAMHSA, 2014; Pinderhughes, Davis, \& Williams, 2015; Ligenza, 2011). For example, a 2008 World Health Organization (WHO) study revealed that " $70 \%$ of all respondents had experienced at least one type" of trauma, including collective violence, interpersonal violence, accidents, and others (Magruder et al., 2016, p. 2). ${ }^{2}$ Responding to this need, SAMHSA crafted a definition of trauma that could

\footnotetext{
${ }^{2}$ See also Benjet et al., 2016.
} 
support the development of a trauma-informed framework for use across sectors and systems:

Individual trauma results from an event, series of events, or set of circumstances that is experienced by an individual as physically or emotionally harmful or life threatening and that has lasting adverse effects on the individual's functioning and mental, physical, social, emotional, or spiritual well-being. (SAMHSA, 2014, p. 7)

Aligning with the importance of cultural responsiveness, SAMHSA (2014) further noted that "communities as a whole can...experience trauma" including (but not limited to) conflict, natural disasters, and ongoing systemic oppression, "often referred to as historical, community, or intergenerational trauma" (p. 17). This was echoed by Pinderhughes, Davis, and Williams (2015), who asserted that "[t]rauma manifests at a community level" (p. 4), and that addressing it requires strategies at the economic, socialcultural, and environment levels. In other words, trauma must be understood as a public health issue relevant to all levels of the social ecological model—an understanding that requires modification of public health education, interventions, policies, and research practices.

The urgency of better incorporating trauma-informed strategies in public health is compounded when one considers the prevalence of Adverse Childhood Experiences (ACEs), which affect over 60 percent of the U.S. population (Kalmakis \& Chandler, 2014; Peters, 2016). While ACEs are not strictly synonymous with trauma, they do regularly result both in trauma (Oral et al., 2016) and in the devastating long-term health consequences associated with it, including developmental disruption, physical and mental illness, risk behaviors, and increased health care usage and costs (Peters, 2016; Kalmakis \& Chandler, 2014). To be sure, the prevalence and long-term effects of ACEs and trauma 
require public health investments in prevention, education, and methods of data collection that respect and respond to individual and community histories. ${ }^{3}$

\subsection{Linking Cultural and Trauma Responsiveness}

It is important to note that cultural- and trauma-responsiveness are inherently

linked. The nature of generational, historical, and community trauma suggests that trauma-informed interventions and research methods require culturally-responsive approaches (Khanna \& Madoori, 2013; Brown \& Strega, 2005). For example, the recommended trauma-informed practice of providing extensive opportunities for choice and control, when applied to public health research, implies the adoption of culturallyresponsive approaches that allow community members to participate and communicate on their terms (SAMHSA, 2014; Peters, 2016). Similarly, a public health strategy that responds to a community's existing communication values and preferences simultaneously reflects "sensitiv[ity] to ways in which [research] institutions may inadvertently re-enact traumatic dynamics" (Khanna \& Madoori, 2013). ${ }^{4}$

In addition, the equitable inclusion of diverse voices in public health researchone aspect of cultural responsiveness in pursuit of health equity — demands that researchers accommodate individuals whose experiences of trauma or stigma may render their participation in traditional studies, formats, and venues unsafe or inaccessible (Baker et al., 2016; SAMHSA, 2014; Bloom, 2018). As Napier et al. (2014) noted, “[w] hen people overdetermine, confine, or reduce care [and research] practices, they not only limit expression of other voices; they also eliminate their own ability to recognise

\footnotetext{
${ }^{3}$ See Pinderhughes, Davis, \& Williams (2015) for a discussion of community trauma under the title of "Adverse Community Experiences."

${ }^{4}$ Notably, Khanna \& Madoori (2013) argue that avoiding potential re-traumatization "requires building cultural competence" (emphasis mine).
} 
[sic] when they have done so" (2014, p. 1625). Finally, given that "some types of trauma are disproportionately experienced by certain groups because of deeply entrenched structural inequalities" (Bowen \& Murshid, 2016, p. 223), the development of traumainformed public health practices should also be seen as a critical aspect of eliminating the systemic, structural, and social barriers to health and well-being.

\subsection{Existing Strategies in the Field}

\subsubsection{Minor Adaptations}

Considerations of culture and trauma have led public health researchers and practitioners to make some modifications, particularly to environmental context and word choice in surveys, focus groups, interviews, and educational initiatives (Nivens, 2015; Johnson, 2016; Leitch, 2017). However, these considerations have yet to lead to a consistent development or uptake of innovative methods related to data collection and health education. For example, a recent SAMHSA guide for trauma-informed practices suggests that interviewers avoid pushing study participants to answer every question; offer several options to ensure participants' sense of control; and make environmental changes that emphasize inclusion and safety (Nivens, 2015). Strikingly, the guide does not propose new methods, nor does it suggest the use or availability of alternative means of eliciting data.

\subsubsection{Participatory Research}

Participatory research strategies, including participatory action research (PAR) and community-based participatory research (CBPR), were designed to challenge the hierarchical and often colonizing nature of traditional research practices by including affected populations in aspects of the research process, and by making action and 
transformation fundamental to the work (van der Riet, 2008). While participatory research "includes a range of techniques" (van der Reit, 2008, p. 551), it generally has contributed research innovation by: critiquing traditional practices as inevitably limited (Evans et al., 2009), insisting on meaningful inclusion and reciprocity (Fine \& Torre, 2006), and offering an alternative to "linear cause and effect" inquiries via a "framework that considers the contexts of people's lives" (MacDonald, 2012, p. 36).

PAR and CBPR were particularly innovative due to their recognition that research practices inevitably act upon communities and populations in multiple ways-even when action is not an explicit goal. Proponents of action research observe that, particularly when conducted among marginalized or vulnerable populations, traditional research approaches often extract time and labor without reciprocity (Evans et al., 2009); stimulate hope for changes that they are not designed to deliver (Ferreira \& Gendron, 2011); and generate outsider representations of communities and populations that may not then be easily altered or contested by members (van der Riet, 2008). In other words, research necessarily acts upon its subjects, and PAR and CBPR approaches sought to build intentionally upon this reality. Proponents of participatory strategies recognized that the research process need not be designed merely to identify and observe; instead, with structured planning and resource provision, the research process could itself actively respond — meaningfully and equitably — to that which is discovered.

Despite these significant innovations, participatory research projects in public health have largely maintained the premises and structures that undergird traditional research practices. As Stanton (2014) observed, PAR and CBPR are often more likely to invite community members into the "epistemological power structure upheld by 
mainstream academia" (p. 576) than to engage in critically innovative processes by which diverse knowledges, communication methods, and meaning-making strategies are utilized, honored, and sustained. For example, a participatory approach may invite community members or service users to advise on survey assessment items or other research tools (see Minkler, 2000), but fail to fundamentally question the effectiveness of itemized assessments in producing knowledge. In addition, while expanded participation in research can increase cultural responsiveness, this is not inevitably the case, and invitations for partnership do not themselves address issues of exclusion such as access, literacy, or trauma-responsiveness. ${ }^{5}$ Indeed, MacDonald (2012) noted that one of the challenges of participatory strategies is the fact that participants do not always feel that genuine communication is safe or feasible. As Bergold and Thomas argued, “[p]articipatory approaches are not fundamentally distinct from other empirical social research procedures" (2012, n.p.).

In sum, at their best, PAR methods in public health have indicated a responsive awareness of the oppressive nature of traditional research practices, the ability of research to stimulate action, and the importance of expanding public health's sources for influence and decision-making (see Israel et al., 2001; Fine \& Torre, 2006). Yet in health-related settings, these methods most typically respond to these awarenesses by inviting greater participation in a dominant method of knowledge production rather than by reconfiguring knowledge-production practices. ${ }^{6}$ To be sure, such an invitation itself marks significant

\footnotetext{
${ }^{5}$ Of course, given the number and variety of PAR-based studies, such issues have certainly been consciously and effectively addressed in many settings and for many populations. Nevertheless, it is always useful to consider the extent to which a method may destabilize researcher-subject hierarchies while maintaining limits on the forms of participation possible, types of data sought, methods of presentation/dissemination offered, etc.

${ }^{6}$ An exception to this is suggested by the photovoice method, noted below. Exceptions can also be seen in other disciplines, including psychology and education, in which visual representations (such as "mapping,
} 
progress in public health practice, but further exploration is necessary to ensure increases in equity, quality data, reciprocity, and health benefits.

\subsubsection{Photovoice}

An example of such methodological exploration in public health is suggested by the PAR method of photovoice: "a process by which people can identify, represent, and enhance their community through a specific photographic technique" (Wang \& Burris, 1997). Photovoice is grounded in Paulo Freire's (2005) argument that visual images of daily experiences can raise critical consciousness - in part by stimulating reflection about the social structures and power dynamics that determine community circumstances. Because the method centers visual images as its means of communication, it challenges textocentric approaches to academic research while also opening the opportunity for participation among groups with varying abilities. ${ }^{7}$ In addition, the use of photographs creates a built-in means of disseminating member-created representations of their life experience, which may productively challenge oppressive outsider representations (Wang \& Burris, 1997). Finally, the fact that photovoice was designed to reach policymakers (Wang \& Burris, 1997; Evans-Agnew \& Rosemberg, 2016) aligns with public health’s increasing attentiveness to social determinants and health in all policies. Frequently used among vulnerable or marginalized populations (Budig et al., 2018), photovoice studies have increased exponentially over the last two decades - indicating both its utility in public health research and the field's lack of similarly promising methods. This popularity combined with the method's untapped potential suggest a ready opportunity for innovation in public health inquiry. In the

modeling, analytic diagramming, photo-voice methods [sic], timelines, trend analysis, and seasonal calendars" (van der Riet, 2012, p. 551)) have been used in data collection.

${ }^{7}$ See Coemans \& Hannes (2017), who argue that "[t]raditional research methods have not always been able to fully engage with... those who have limited language abilities or those who suffered major traumas or severe life circumstances that are difficult to verbalize" (p. 35-36). 
current study, photovoice functions as an initial model for prototypes of culturally responsive, trauma-informed research methods that respond to multiple levels of the social ecological model.

\subsection{Opportunities to Make Advances}

Like participatory research more generally, photovoice is not without its criticisms and limitations. Most obviously, it has often failed to initiate the policy or social changes it promises to address; it has also been inconsistent in its ability to deal transparently with researcher-driven goals and with ownership and consent issues related to photographs. ${ }^{8}$ Photovoice study authors have also often been quick to label the method "empowering" for participants, while largely failing to develop theories and studies explicating this and other potential direct health benefits. Similarly, there seem to be widespread assumptions in public health about the cultural relevance of photography as a means of expression, regardless of the population or community in question. Finally, photovoice has been unnecessarily limited by public health researchers' failures to more robustly extrapolate its lessons regarding: 1) the potential value of arts-based methods in health research and interventions, and 2) the ability of arts-based practices to enhance policy advocacy, press and promotion, and public interaction.

The current study recognizes these limitations as opportunities to make immediate improvements in public health methodology. In particular, by drawing upon theories from multiple disciplines_critical and intersectional theories, expressive writing and arts-in-health research, hermeneutic theory, and design thinking — this project builds upon photovoice in order to structure new research questions and a pilot study regarding trauma-informed, culturally-responsive public health inquiry. Because a foundational

\footnotetext{
${ }^{8}$ These and other criticisms are discussed in Chapter Two.
} 
understanding of these theories is necessary for the conceptualization of the current study, a brief overview of each is offered below.

\subsection{Theoretical Foundations}

\subsubsection{Critical Theory and Intersectionality}

At root, critical theories seek to critique and alter society, rather than simply to understand or explain it — the latter of which is typically the task of traditional theories (Geuss, 1981). For example, a critical theoretical take on scientific practices questions the possibility of neutrality or objectivity, noting that science is, like most systems, constructed via the prioritization of specific values and ways of knowing that have historically privileged specific persons, values, and populations (see Ford \& Airhihenbuwa, 2010; Brown \& Strega, 2005; Napier et al., 2014). ${ }^{9}$ As a result, scientific practice has often marginalized or erased knowledges and lived experiences that lay beyond its scope: placing them "low down on the hierarchy, beneath the required level of cognition or scientificity" (Foucault, 1980, p. 82). ${ }^{10}$ In response, critical theory argues for ongoing reflection on and analysis of the ideologies underpinning social and scientific practices, as a necessary precursor to determining — and then taking_liberating action (Foucault, 1980; Horkheimer, 1982).

Critical theory is a necessary frame for the advancement of health equity, because it calls scientists to acknowledge the extent to which the embedded prioritization of specific methods, epistemologies, practices, and competencies in public health have

\footnotetext{
${ }^{9}$ In fact, Napier et al. (2014) noted that "biomedical knowledge is itself an artifact and outcome of cultural practices" (p. 1624).

${ }^{10}$ According to Foucault (1980), researchers can resist such subjugation by supporting the "re-emergence" of formerly "low-ranking knowledges" (p. 82). When such knowledges are included in historical and social analyses, scientists are better equipped to develop "a painstaking rediscovery of struggles" (Foucault, 1980, p. 83) — which is essential to the promotion and realization of health equity.
} 
maintained and potentially exacerbated health inequities by requiring that health, health care, communication, adherence, retention, and health behaviors be defined, understood, and valued according to dominant ideologies and cultural norms. ${ }^{11}$ In response, public health researchers and practitioners must "understand that there are many different kinds of knowledge"' (Knight, 2014, p. 193). Indeed, health equity will not be realized unless and until the field develops methods of equitably eliciting and valuing diverse knowledges. $^{12}$

Intersectionality theory, developed by Kimberlé Crenshaw, particularizes this critical theoretical lens by noting the need, when discussing power structures and oppression, to consider all axes of power: race, gender, sexual orientation, ability, and more (Cho, Crenshaw, \& McCall 2013). According to Crenshaw (2018), the overarching intersection of these potential points of discrimination is greater than the sum of each of them; because of this, discussions of equity require not only feminist and critical race theories (for example), but a theory of intersectionality that recognizes and attempts to address the compounding effects of multiple forms of oppression.

\subsubsection{Application}

Intersectionality theory is fundamental to health equity efforts; without recognizing multiple and intersecting experiences of oppression, the field cannot adequately recognize the social determinants of adverse health outcomes—or adequately respond at relevant levels of the social ecological model. Particular to the current study, intersectionality theory in research design calls upon scientists to be aware of ways in

\footnotetext{
11 "Cultural norms" here refers to Western cultures as well as the "smaller" cultures of academia, science, publication, institutional policy and practice, funding processes, etcetera.

${ }^{12}$ As indicated above, this involves trauma- and cultural-responsiveness, so as to actively remove barriers to access, inclusion, and communication.
} 
which research designs, epistemological assumptions, and standardized practices may be or become differently or multiply exclusionary, affecting varying target populations (and individual participants/respondents) in varying ways.

The development of effective, responsive methods will require surrendering the possibility that practitioners can merely "import specific, fixed intervention protocols" into population health initiatives (Trickett et al., 2011, p. 1412), or that we can unreflectively "privilege quantitative knowledge over qualitative knowledge" (p. 1414). Such approaches not only privilege and exclude particular knowledges and populations, but they (therefore) risk the distortion of data and findings, thwarting scientific progress and improvements to health equity. As Napier et al. (2014) argued, "[e]mphasis on data more than basic human interaction ... contributes not only to devaluation of personal meaning, but also to widening of health disparities" (p. 1626). Indeed, Foucault (1980) brought a critical theoretical lens to the pervasive quantitative/qualitative divide when he pointedly asked, "What types of knowledge do you want to disqualify in the very instant of your demand: 'Is it a science?'” (p. 85).

Critical and intersectionality theories not only inform and support efforts to effectively and ethically address health equity, but they also help illuminate the fact that research values and practices are themselves among the social determinants of health and health equity. As such, they demand interrogation, analysis, and innovation.

\subsubsection{Expressive Writing and Cognitive Change Theory}

Given the reliance of data collection upon disclosure (in varying forms), the current project also draws upon research regarding the impacts and mechanisms of disclosure - including the results of expressive writing studies. "Expressive writing" as a 
practice entails writing reflectively about one's thoughts and feelings regarding traumatic experiences, particularly those that have not yet been disclosed. Frattaroli (2006) noted that, after experiencing "an important life event, the tendency to disclose information about that event has long been considered both normal and healthy" (p. 823). Over decades of study, expressive writing research has affirmed this tendency and linked it to health (Pennebaker, 2006) —with hundreds of studies revealing consistent, significant well-being outcomes from expressive writing. These outcomes included decreased depression, fewer post-trauma symptoms, improved immune function, and better grades ${ }^{13}$ importantly, outcomes also included participants' newfound ability to communicate about issues they had not yet shared with anyone (Bolton, 2007; Kearns et al., 2010; Pennebaker \& Seagal, 1999).

Researchers have hypothesized that "the mechanism by which expressive writing is helpful" (Frattaroli, 2006, p. $825^{14}$ ) is cognitive change theory (Alparone, Pagliaro, \& Rizzo, 2015). Cognitive change involves "making sense of an event, gaining insight about a trauma, and...organizing and integrating an upsetting experience into one's selfschema" (Frattaroli, 2006, p. 825); notably, it aligns with narrative theories that have shown "re-storying" to be "a key to...collective transformative action" (Goodson \& Gill, p. 98). Expressive writing studies have contributed to a growing recognition that mental and physical well-being are reliant upon disclosure, narration, and the ordering and processing of difficult experiences (Pennebaker \& Seagal, 1999; Ramírez-Esparza \&

\footnotetext{
13 These benefits have been noted among diverse participants, including "grade-school children and nursing home residents, arthritis sufferers, medical school students, [male] maximum security prisoners, new mothers, and rape victims" (Pennebaker, 2012, p. 40).

${ }^{14}$ See also Alparone, Pagliaro, and Rizzo, 2015.
} 
Pennebaker, 2006; Swanbon, Boyce, \& Greenberg, 2008; Nixon et al., 2015; Dalgaard \& Montgomery, 2015).

\subsubsection{Application}

Adding expressive writing and cognitive change theory to the preceding discussion of trauma- and cultural responsiveness, the current study argues for the potential public health value of expanding mechanisms for disclosure among study participants. Such expansion may not only provide direct health benefits to participants (as seen in expressive writing studies), but it may also improve public health initiatives by: 1) providing data that may otherwise remain unshared; 2) helping normalize the sharing of difficult experiences, thereby reducing stigma; and 3) initiating a process of improved community health via improved communication and new potential connections (see Zoughbie, 2013; Christakis \& Fowler, 2009).

\subsubsection{Arts in Health Research}

The development of public health methods that build upon expressive writing benefits can be supported and informed by the arts-in-health literature. While reviewing this literature is beyond the scope of this paper, ${ }^{15}$ art's potential to enhance health communications-particularly as this enhancement relates to stigma reduction, cultural responsiveness, and social connectivity—is fundamental to the current project. ${ }^{16}$

For example, the literature demonstrates that art can adeptly challenge stigma related to mental health, HIV/AIDS, trauma, and other health issues via several

\footnotetext{
${ }^{15}$ For introductory material regarding arts and well-being, see Hanna, Rollins, \& Lewis, 2017; Stuckey \& Nobel, 2010; Bygren, Konlaan, \& Johansson, 1996; Clift \& Camic, 2016; Clift, 2012.

${ }^{16}$ Davies et al. (2016) argued that "arts engagement...has a positive impact on mental, social and physical wellbeing, and is a versatile means of improving health literacy, access and creating health benefits for the general population and vulnerable groups" (p. 304).
} 
mechanisms: educating the public, providing or increasing contact with individuals experiencing the stigmatized issue, reducing feelings of isolation, discussing difficult topics in approachable ways, altering social norms and perceptions, or protesting actions or policies that perpetuate biases (Gronholm et al., 2017; Hand \& Golden, 2018; Tsiris et al., 2011; Ings, Crane, \& Cameron, 2012; Rollins et al., 2011). Though art's specific applications have varied widely across several stigma-related issues, art has consistently rendered the process of stigma reduction engaging, memorable, inclusive, and traumainformed. ${ }^{17}$ As such, exploration of arts-based communication methods for additional public health purposes — such as data collection — is warranted.

Arts-based practices have also helped initiate or sustain relationships among community members, researchers, policymakers, and other local leaders, in part because art "gives people a voice,"18 "encourages self-expression," and "improves communication and strengthens relationships" (Kilroy et al., 2007, p. 19). Sociologist Stephen Duncombe (2016) additionally notes that art may increase civic engagement — which is necessary for advancements in public health policy and health equity—by functioning as political expression and issue education; creating space and occasion for local dialogue (an identified dimension of community capacity); "creating disruption" in a community (p. 122); articulating new community visions; or amplifying local realities. Such public communications potential—along with art's ability to "express issues and solutions" in

\footnotetext{
${ }^{17}$ Of course, it realizes these benefits to varying degrees.

18 This author contests the notion of "giving" others "a voice," because all individuals have a voice (understood as thoughts, ideas, opinions, desires). The challenge, particularly for public health, is therefore in ensuring that individuals have opportunities to speak to power regarding their own health and well-being and that, when they do, they are heard and seriously considered. To Kilroy et al.'s point, however, it is important to note art's ability to bring attention — via increased interest, empathy, awareness, media coverage, et cetera - to voices that are otherwise excluded, minimized, or muted in conversations related to policy, health, and well-being.
} 
powerful, memorable ways - are why Ombler and Donovan (2017) argued that collaborating with artists regarding public health messaging and dissemination is a critical aspect of addressing the field's most pressing challenges. ${ }^{19}$

Regarding cultural responsiveness, studies have illuminated the role of arts practices in helping community members assert and nurture cultural identity (Muirhead \& Leeuw, 2012; Newman, Curtis, \& Stephens, 2003), a positive sense of which is linked to well-being (Congress Lowitja, 2014; Shepherd, Delgado, Sherwood, \& Paradies, 2018). By more intentionally considering and incorporating cultural identities and practices in their health initiatives and communications (such as by including the arts), researchers can improve interactions, relationships, engagement, and effectiveness of health promotion efforts. In addition, by observing and analyzing a given population's existing arts practices and products, researchers stand to learn a great deal about that population's values, needs, assets, and priorities.

Finally, arts-based practices have been shown to increase social connectivity and community interactivity. These are linked to well-being (Seppala, 2014; Saeri, Cruwys, \& Barlow, 2017; Ferris, 2012; Umberson \& Montez, 2010), in part because they generate spaces and occasions via which residents meet and communicate with one another (see Ings, Cran, \& Cameron, 2012; Muirhead \& Leeuw, 2012; Parkinson \& White, 2013; Clift, 2012; Newman, Curtis, \& Stephens, 2003). Such spaces and occasions can also serve the purposes of mutual learning (with researchers, policymakers), providing access

\footnotetext{
${ }^{19}$ See also fields related to public health communication goals, including social marketing, media activism, cultural organizing, and the use of art in social movements. An introduction to these fields can be found in Duncombe et al., 2017. Notably, Camic (2008) presaged this argument, noting that the arts "make health education (and promotion) more memorable and enjoyable... and more likely to engage a target population" (p. 292).
} 
to services, or community organizing for specific concerns. In sum, arts- and creativitybased initiatives have demonstrated their significance in meeting health and health communication goals; as a result, their use in public health research warrants continued development.

\subsubsection{Hermeneutic Theory}

Hermeneutic theory is based on the idea that "the most basic fact of social life is the meaning of an action" (Little, 2008) — with the word action here referring to behaviors, speech acts, decisions, community activities, politics, and more. ${ }^{20}$ Hermeneutic theory posits that we cannot understand an individual's or group's behaviors without understanding 1) the meaning these behaviors hold for the actors themselves, and 2) the meaning those actors made of actions and circumstances that came before their own behavior. In this sense, hermeneutic theory models a root-cause approach through the lens of meaning: linking individual behaviors and population-level inter-activities through a layered process of communication and interpretation.

\subsubsection{Application}

Hermeneutic theory contributes to the current study by asserting that meaningmaking across difference is a primary aspect of social reality; ${ }^{21}$ as a result of this, methods that fail to capture or translate modes of meaning-making can neither adequately assess current circumstances, nor adequately inform responsive action. This is particularly the case in research related to health equity or social determinants, in which

\footnotetext{
20 The theory's origins lie in the interpretation of ancient texts; in that context, hermeneutics has referred to the process of attempting to uncover the authentic, original meaning of a text, or even its potential hidden meanings (Bauman, 1978). Hughes and Sharrock (1997) describe hermeneutics in social science as the process of "developing valid methods for understanding others, especially those from whom we are historically and culturally remote" (p. 20).

${ }^{21}$ In the social sciences, obtaining a reliable, "correct" interpretation may be seen as a less reasonable or meaningful goal than developing understandings regarding others' texts.
} 
the variables under examination are often socially constructed. In addition, hermeneutic theory suggests that differences among communicative mechanisms and meanings are to be expected — and even sought for study — rather than avoided (or manipulated into homogeneity) via the use prescribed, ostensibly neutral communicative devices. ${ }^{22}$ Finally, hermeneutic theory suggests that knowing how to find, elicit, co-create, analyze, interpret, or disseminate multiple modes of communication and meaning-making may render better understanding — at several levels of the social ecological model — than aiming for simplistic cause and effect explanations. Notably, this more expansive approach also generates greater potential for trauma- and culturally-responsive practice.

\subsubsection{Design Thinking and Systems Theory}

The present study also draws upon design thinking and systems theory to develop trauma-informed, culturally-responsive methods of inquiry and data collection. First, systems theory as applied to public health recalls the social ecological model; it acknowledges that individuals' health is determined by "multiple interrelated systems" (Nguyen, 2015). Trickett et al. applied systems theory to community health interventions by "emphasiz[ing] the permeating role of culture and cultural history as both a resource for and an influence on the community-intervention process" (p. 1413, emphasis mine). In other words, systems theory moves beyond culture, identity, and expressive practices as social determinants or influencers of health, to the recognition that these practices also often describe or express health. This recognition indicates the necessity of expanding sources of health data to include those rooted in expressive and cultural practices.

\footnotetext{
${ }^{22}$ Such as surveys, screenings, or even guided interviews.
} 


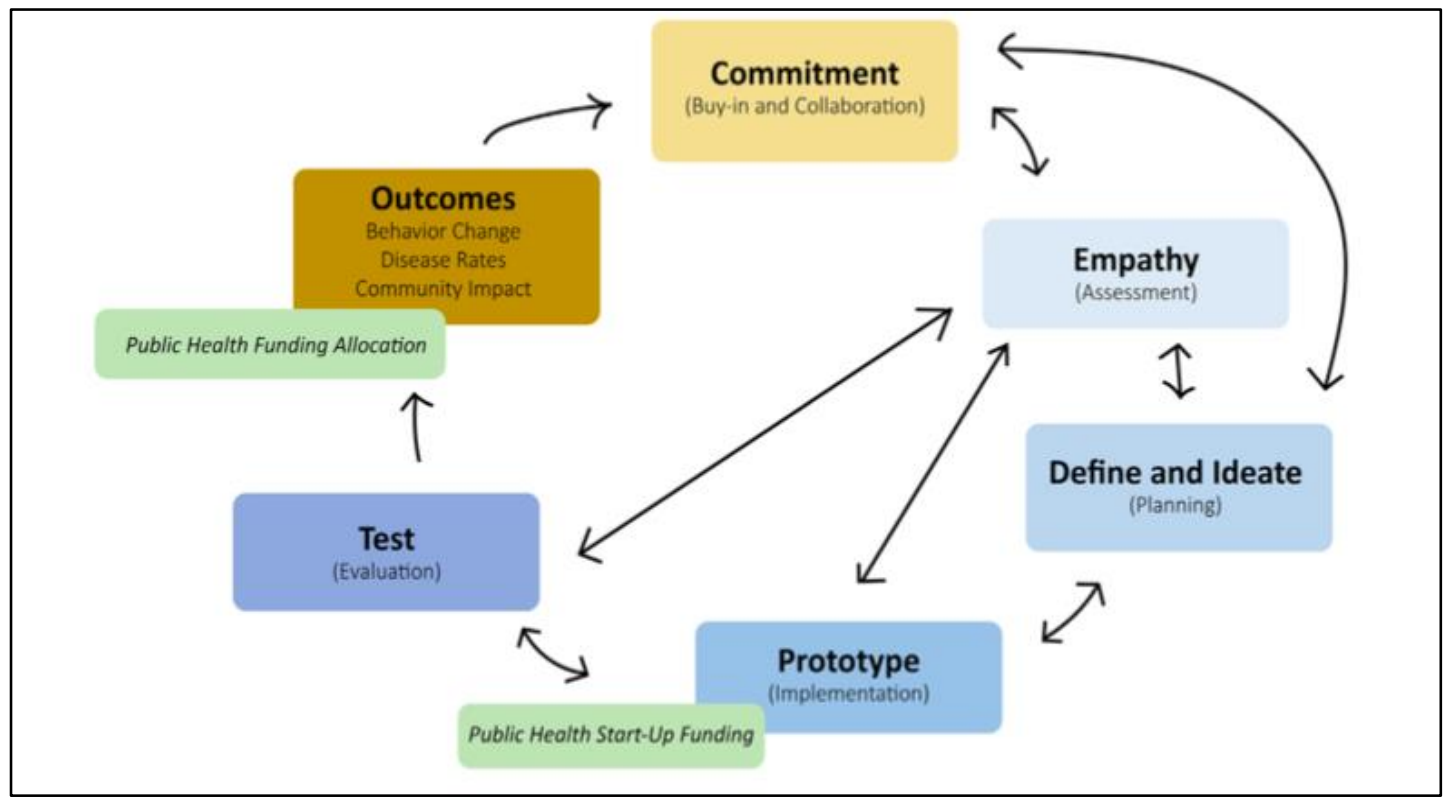

Figure 1.1: Public Health Innovation Model (Lister et al., 2017, p. 4)

Second, reflecting Lister et al.'s (2017) Public Health Innovation Model (presented in Figure 1.2), this project innovates based upon design thinking. For example, the Commitment stage of Lister's model is embodied by this study's commitment to culturally-responsive, trauma-informed strategies in public health that attend to inclusivity, cultural sustainment, and communicative choice. Similarly, the Empathy stage informs this study's "human-centered approach" (Lister et al., 2017, p. 6), including an emphasis on lived experience, narrative, and "checking cultural biases" (p. 6). The project incorporates the Define and Ideate stage by “'step[ping] beyond obvious solutions"” (quoted in Lister et al., 2017, p. 6) to experiment with new sources of useful data. This includes heeding Lister et al.'s (2017) caution against over-relying on evidence-based practice, instead elevating "autonomy and creativity... when addressing the new or unfamiliar" (p. 6). 


\subsection{Synthesis of Theories}

The above theories, frameworks, and bodies of research, in combination with the strengths and untapped potential of photovoice (discussed earlier and in Chapter Two), invite an interrogation of the adequacy, utility, and equitable nature of conventional public health research practices. They also suggest the potential value of: expanding expressive options in data collection; increasing trauma- and cultural-responsiveness, particularly among marginalized populations; and further emphasizing critical reflection in research practices, designs, and assumptions. These interrogations and suggestions generated two research questions related to public health research methods, which now form the foundation of this study: First, What does a population's art reveal about its health, needs, assets, values, and priorities? And second, how do arts-based data and data collection practices compare to those associated with conventional forms of inquiry—particularly with regard to accessibility, inclusion, participant risks/benefits, and opportunities for public education or awareness?

To begin answering these questions, the researcher continued moving through the stages of the Public Health Model of Innovation (Lister et al., 2017). During the Prototyping process, the decision was made to use small local(ized) artistic data sets to assess needs, values, beliefs, assets, and priorities related to a stigmatized or potentially traumatizing issue; in this study, that issue was violence. The goal was to develop methodological prototypes that would, in Lister et al.'s terms, "nail the pain"- getting at a precise, identified need - "as opposed to creating full-scale, error-free products that are expensive and require long development cycles" (Lister et al., 2017, p. 7). In other words, the methods developed and analyzed in this study were designed not only to examine the 
value of new research strategies, but also to offer relatively rapid enactment and evaluation of those strategies - as a model for continued innovation in public health inquiry. ${ }^{23}$

\subsection{Authorial Positionality}

Given the critical theoretical framing of this project, and its argument that ways of knowing are inevitably affected by values, histories, and observer positions, it is important to note the author's own position in the current study. This author comes to research in public health and health promotion following a career in the arts, during which writing and performing songs about difficult experiences helped stimulate and sustain her health. More importantly, on tours around the globe, listeners regularly stayed after shows to share with the author their own experiences with similar issues-often disclosing these experiences for the first time. Listeners often attributed their openness to their sense that the author's public disclosures via song had served to reduce stigma. Others made use of the songs' metaphors when disclosing their own experiences, which appeared to allow communication of otherwise inarticulable histories. These personal and public occurrences, combined with the researcher's academic backgrounds in music, creative writing, and rhetoric, cultivated an interest in and alertness to creative arts as means by which humans make meaning, communicate identities and experiences, and challenge stigma and norms.

In addition to career histories, the author has facilitated creative writing workshops with incarcerated girls for several years. This population is consistently

\footnotetext{
${ }^{23}$ Given its accessibility and potential for engagement and participation, arts-based inquiry may be particularly amenable to prototyping and responsive development — which is an additional benefit of its inclusion among options for public health research design.
} 
underserved by a system historically designed for males (Sherman \& Balck, 2015); in addition, the disproportionate representation of girls of color and LBT/GNC ${ }^{24}$ girls in this population (Golden, 2017)—combined with the often re-traumatizing experience of incarceration (Sherman, 2005) — regularly highlighted the author's privilege as a free, white, cis-gender heterosexual woman. Therefore, cultivating and sustaining relationships with workshop participants required ongoing education and critical reflection regarding race, (in)justice, privilege, and power structures, as well as the intersectional experiences, oppressions, and sociocultural contexts that determine levels of health (or lack of it) among justice-involved girls. This process informed and reinforced the emphases placed on these issues in the current study.

Work with incarcerated young women additionally demanded ongoing experimentation to stimulate and support effective communication across differences (culture, race/ethnicity, power/access, age, etc.). This history of experimentation informed the incorporation of design theory into this study, including the use of prototypes. Finally, the author observed differences in girls' communications (content and affect) when they wrote about their experiences versus when they discussed them; similarly, their writing and conversation in the creative intervention space differed from ways in which participants spoke or responded to non-intervention settings and staff. These communicative disparities contributed to the author's recognition that creative, adaptable options may be necessary if health research is to more effectively include the knowledges of persons who have been oppressed, marginalized, or traumatized.

\footnotetext{
${ }^{24}$ Lesbian, bisexual, transgender, and gender non-conforming.
} 
It would have been impossible to avoid bringing these experiences and knowledges to the inquiry at hand, as they inevitably informed the significance placed by the current study on the potential roles of creative expression, trauma histories, intersectional oppression, and communicative options. They are thus brought with intention and awareness, as the field's inability to respond consistently or adequately to trauma and to cultural difference-and the consequences of such failures for health and health equity — demand that researchers take the risks associated with our inevitable subjectivity. Ongoing health inequities demand that researchers apply scientific curiosity not only to identified objects of research, but to the assumption that objectivity is possible, or that objectivity should be elevated over transparent reckoning with the exclusionary means by which scientists have recognized, accessed, valued, assessed, documented, and disseminated knowledges. Researchers' denial or obfuscation of the value-laden nature of conventional inquiry has not prevented us from participating in the power structures inherent in scientific study; instead, denial has merely institutionalized and perpetuated that participation. It is therefore hoped that the theoretical frameworks and researcher transparency grounding this introduction serve to productively destabilize research conventions: stimulating generative discomfort, meaningful innovation, and ongoing critical reflection.

\subsection{Dissertation Overview}

Chapter Two of this dissertation discusses the social ecological and biomedical models in public health, problematizing epistemological strictures that preclude the recognition, elicitation, and analysis of diverse knowledges - and thus generate inadequate or misguided data, resources, and practices. Chapter Two then expands upon 
the discussion of photovoice already offered: reviewing that method's limitations and untapped potential, and utilizing the current chapter's critical frameworks to consider new methodological approaches that build upon photovoice.

Grounded in Chapter Two's literature review and methodological considerations, Chapter Three introduces the means by which new arts-based approaches will be utilized in the current study for the purposes of public health inquiry. Keeping to the goal of rapid prototype creation and evaluation, Chapter Three defines three populations, describes seven unique data sources (one arts-based and one or two quantitative sources for each sample), and details how these will be analyzed. Chapter Four then presents the results of this analysis, while Chapter Five features a discussion of all findings, including strengths and limitations of utilized methods, and recommendations for further research. 


\section{CHAPTER TWO: LITERATURE REVIEW}

Foundations for Innovation

If the field of public health is to advance health equity, it must examine existing epistemological strictures that preclude the recognition, elicitation, and analysis of diverse knowledges, thus perpetuating inequitable value structures and the marginalization of many populations and issues relevant to public health. At the least, to the extent that public health inquiry fails to acknowledge its limited epistemological assumptions, it will continue to generate inadequate or misguided data, resources, and practices.

This chapter will therefore review some of the challenges presented by overreliance upon - and overconfidence in - traditional methodologies, particularly among marginalized, over-researched, and vulnerable populations. Drawing upon Chapter One, this chapter will also consider public health's attempts to address these challenges, including via participatory action research strategies such as photovoice-which is noteworthy due both to its exponentially-increasing popularity and its status in public health as a go-to method for creative, image-based data elicitation and analysis. This chapter's examination of photovoice provides background about the method as well as several critiques, including its prominence in public health to the exclusion of similar

methods. The chapter closes by drawing upon theories noted in Chapter One in order to 
discuss photovoice's untapped potential—including as a trauma-informed, culturallyresponsive method of data collection — and what it suggests about the value of creative strategies for knowledge production, inclusion, elicitation, and analysis in the field of public health.

\subsection{Conventional Methodologies: Hegemony and Inequity}

As mentioned in Chapter One, a discussion of methodological examination and innovation in public health must be grounded in an understanding of the field's recent and incomplete shift from a biomedical to social ecological approach to health. The recognition within public health of ways in which social, community, systemic, cultural, and historical factors affect health—each of these indicated by levels of the social ecological model—has led to increased efforts to reduce persistent health disparities caused by such factors. Indeed, the field's shift to a social ecological approach implies a critique of the driving principles of the biomedical model,${ }^{25}$ including foundationalist ontology, positivism (accompanied by empiricism), reductionism, dualism, and the assumption that all illness can be traced to-and treated via—-specific pathologic origins (see Adibi, 2014). Unfortunately, the field of public health has yet to make this critique consistently explicit; the field's investment in the social ecological framework has yet to generate consistent, open dialogue and reflection regarding the driving principles of earlier paradigms.

Among the explanations for this gap in dialogue and critique are the many structural barriers to pursuing diverse knowledges and knowledge-producing methodologies in the field of public health. For example, funding structures and

\footnotetext{
${ }^{25}$ At the least, it implies their inadequacy.
} 
requirements regularly pre-determine the means by which researchers and practitioners conduct inquiries, often by emphasizing scalability and evidence-based methods (Okagaki \& Dean, 2016; Jacobsen, Butterill, \& Goering, 2004). Similarly, the academic imperative to publish requires that researchers adhere to the publication standards established by editorial boards which, in public health and behavioral sciences, have long held a bias toward quantitative studies (Janßen, Sauter, \& Kowalski, 2012). These structural realities preclude the ongoing innovation necessary to improve access and responses to diverse knowledges and meaning-making practices. Ultimately, they perpetuate a fear-based cycle of limited innovation and inadequate research practices, and their consequences: continued researcher intrusion, potential re-traumatization, underrepresentation of populations and issues, ${ }^{26}$ culturally inappropriate practices, deficit-centered interventions, and more.

It is clear that, to the extent that it fails to consciously interrogate and adapt the epistemological and ontological foundations of biomedicine and traditional health approaches, public health cannot execute an adequate response to the social ecological perspective. ${ }^{27}$ Researchers and practitioners are likely instead to layer new concepts ("root causes," "social determinants," "health in all policies," "systemic oppression") onto a biomedical paradigm — attempting to answer questions presented by the social ecological schema with methods and assumptions that were developed in response to (and in accommodation of) biomedicine. This approach limits the field's ability to

\footnotetext{
${ }^{26}$ Coemans \& Hannes (2017) argue that " $[\mathrm{t}$ ]raditional research methods have not always been able to fully engage with particular groups in society. This is certainly the case for vulnerable people, for instance those who have limited language abilities or those who suffered major traumas or severe life circumstances that are difficult to verbalize" (p. 35-36).

${ }^{27}$ Indeed, Brown and Strega (2005) note that "research cannot challenge relations of dominance and subordination unless it also challenges the hegemony of current research paradigms" (p. 10).
} 
develop broadened conceptions of well-being, to address a history of inequitable valuation of knowledge and culture, or to effectively advance social and policy changes that increase health equity.

Chapter One noted that the epistemological strictures of biomedicine limit public health's access to diverse knowledges, the accessibility of research findings by varying populations, and the field's ability to challenge power structures (including knowledge hierarchies) that perpetuate inequity and poor health. Responding to the need for dialogue and reflection regarding these assumptions, the following section examines the driving principles of biomedicine, with the goal of illuminating opportunities for methodological and definitional innovation for the advancement of health equity.

\subsection{Principles of Biomedicine}

To begin, the biomedical model is rooted in a foundationalist ontology, "in which the world exists independently of our knowledge of it" and in which "unambiguous and accurate knowledge of the world which can be arrived at through sensory experience" (Houghton, 2011, p. 1). This ontology gives rise to positivist epistemology, which sees the obtainment of "hard, secure objective knowledge" (Carson, Gilmore, \& Perry, 2001, p. 6) as not only possible but singularly valid (see Houghton, 2011). This epistemological approach has driven empiricism: a focus on observation, with the assumption that repeated observation generates reliable, objective knowledge.

The biomedical model also relies on a Cartesian separation of mind and body in which the body is viewed as a manifestation of objectively-observable causes and effects (positivism), while the mind — and "human experience"—-is viewed as subjective and 
thus unreliable, and thus minimally relevant to legitimate knowledge production and the advancement of health (see Rigg, Cook, \& Murphy, 2014).

Grounded in dualism's circumscription of experience and perception, and on positivism's emphasis on observable cause and effect, biomedicine has defined health as the absence of illness (Ashcroft \& Van Katwyk, 2016), tracing illness almost exclusively to specific pathologic origins that can be affected by medical intervention at the individual and cellular levels (Adibi, 2014). This model led to significant technological and pharmacological innovations, ${ }^{28}$ and it has long been effective in combating infectious diseases. However, biomedicine's successes have also served to entrench the model as the standard in health care, including in public health practice. This has remained the case despite biomedicine's inherent reliance on reductionism (see Longino \& Murphy, 1995), on concepts of normality, and on individual physiology and medicalization-often to the neglect or intentional exclusion ${ }^{29}$ of the impacts of social interaction, systems and resources, culture, and other contextual factors featured by the social ecological approach.

This entrenchment of biomedicine's conception of health and illness has influenced and severely limited public health practice, driving the field's focus on treatment and prevention at the individual and clinical levels (PAHO, 2011), ${ }^{30}$ and its related attachment to experimental design as the gold standard for public health

\footnotetext{
${ }^{28}$ Notable advances early on include the development of diagnostic instruments "such as the stethoscope, ophthalmoscope, laryngoscope, microscope, and X-ray" (Ashcroft \& Van Katwyk, 2016, p. 143).

${ }^{29}$ Such as in clinical trials.

${ }^{30}$ Note in PAHO's document that distinctions are made between individual and population levels of intervention, yet population recommendations tend to center individuals' uptake of behavioral, biomedical solutions. Notable exceptions include mentions of policy change.
} 
research. ${ }^{31}$ In short, despite its recognition of social determinants of health, the field's predominantly-accepted understandings of what is knowable - and how it can be reliably known - has determined the limited levels of the social ecological model at which public health actually chooses to invest, operate, and assess its work.

\subsection{Critiques of Conventional Approaches}

In Research as Resistance, Brown and Strega (2005) question the ability of research methods that are grounded in positivist epistemologies to effectively address oppressive systems, policies, and social conditions, because they are themselves grounded in oppressive and inequitable structures. They argue that

[f]raming the discussion about what constitutes knowledge within the discourse of positivism obscures important questions about how the development of knowledge is socially constructed and controlled, how knowledge is used, and whose interests knowledge serves...[I]t fosters an illusion of neutrality or objectivity that has come to be institutionalized... as the standard by which truth claims are assessed. The racialized and gendered foundation of the Enlightenment epistemology that quantitative and qualitative approaches share is [thereby] rendered invisible, and truth claims are sequestered from questions of power, politics, and survival. (Brown \& Strega, 2005, p. 6)

In other words, the institutionalization and standardization of such methods as randomized controlled trials does not render them unaffected by assumptions regarding that which can, should, or will be investigated, argued, or "reliably" known. ${ }^{32}$ And given

\footnotetext{
${ }^{31}$ Randomized controlled trials tend to function adequately for the natural sciences, but cannot accommodate social interaction and context. Al-Azri (2012) argues that the gold standard in research "is built to enhance the existing assumptions and beliefs of this [positivist] paradigm" (p. 256). Rigg et al. (2014) note that, "[i]n the health field in particular, applying the 'gold standard' randomized control trial, without including other approaches, decouples the medical from the human and may result in complete irrelevance to everyday life and the implementation of health interventions" (p. 3).

${ }^{32}$ Indeed, Napier et al (2014) asserted that " The idea that biomedicine is able to address the ills of the world is [itself] a fundamentally cultural notion" (p. 1618), and that "biomedical knowledge is itself an artifact and outcome of cultural practices" (p. 1624).
} 
that such assumptions are inevitably made within or in response to biased and inequitable systems, societies, and institutions, they require critical reflection and interrogation in service of increasingly inclusive — and increasingly adequate — sources of knowledge and knowledge practices.

In addition to risking the perpetuation of inequity or oppression generally, positivist approaches in public health research may also risk elevating explanation-such as a simplified, linear cause and effect-over understanding. As alluded to in Chapter One's discussion of hermeneutic theory, elevating explanation over understanding leads researchers to separate social phenomena from their concomitant sociocultural meanings, including historical narratives and systemic influences, which are variously experienced and therefore variously influential with regard to health. ${ }^{33}$ Conflating explanation and understanding is particularly dangerous when researchers from a dominant population, or who possess a privileged status, seek knowledge from or about communities or populations that have been marginalized, stigmatized, or oppressed. In such situations, the ways in which study participants' histories, perceptions, experiences, and meaningmaking practices influence their health and health behaviors may not be noticed, acknowledged, or adequately understood ${ }^{34}$ — particularly if researchers are not employing methods specifically designed to "understand others, especially those from whom we are historically [or] culturally remote" (Hughes \& Sharrock, 1997). Sociologists John Hughes

\footnotetext{
${ }^{33}$ Note here the importance of having means by which to identify, acknowledge, and analyze the ways in which people make meaning across chronological, sociocultural, and geographical difference(s).

34 The author recognizes that such factors are unlikely to ever be, in the fullest sense, "correctly" or "adequately" understood; however, correct and adequate understanding should be sought to the extent it is achievable at least for the purposes of the study in question.
} 
and Wes Sharrock (1997) figured this concern into their discussion of differences between the natural and social sciences:

[I]t is possible to argue that the problems of the social sciences are much closer to the problem of attaining a reciprocal understanding in a conversation than they are like those of the natural scientists seeking to attain exceptionless generalisations [sic] for natural phenomena. That is, the methodological problems and solutions for the social sciences are of a kind involved in comprehending difficult or obscure communications and not of the sort involved in attaining valid statistical generalisations [sic]." (Hughes \& Sharrock, 1997, p. 20).

Unlike traditional epidemiological and biomedical studies, which are typically understood to fall within the natural sciences, considerations of health disparities, systemic inequities, and the social determinants of health clearly require reckoning with the philosophical and methodological problems associated with the social sciences. These include: debates over the extent to which methods designed for the natural sciences can or should be applied to the study of social phenomena; how studies will measure or address emergent properties that arise out of social interaction, since these are irreducible to individual study; and how to philosophically frame — and scientifically manage — the inevitably subjective work of creating and interpreting indicators when conducting variable analyses. ${ }^{35}$

The "solution" to such concerns is not to discard or demonize particular methods, nor is it to simply shift methodological domination in the field to a new or currently under-used practice. Instead, given the highly contextualized nature of health represented by a social ecological approach, public health must proactively ensure widespread training in, use of, and innovation toward diverse knowledge-producing practices that are up to the challenges of: navigating subjectivity and irreducibility; interrogating the

\footnotetext{
35 This is not meant to suggest that similar problems and considerations do not exist in the natural sciences.
} 
assumptions of science as itself a socio-cultural phenomenon (see Hughes \& Sharrock, 1997); and applying hermeneutic theory to both implicit and explicit socio-culturohistorical narratives.

Once again, the now-extensive uptake of the social ecological approach in public health suggests the field's urgent need to interrogate how public health's biomedical foundations, epistemological assumptions, entrenched knowledge hierarchies, and institutionalized methodologies preclude social ecological responses. The social ecological approach challenges not only biomedicine's focus on the individual, but its assumption that reality — including health - exists independently of social dynamics, institutional and state decisions, and historical injustices. Indeed, at the heart of social determinants and "health in all policies" is a recognition that illness and health are determined not only by pathogens or medicine, but by subjective and dynamic interpretations of human value, social norms, risks and rewards, access and opportunity, state priorities, political expediency, felt moral imperatives, and more —all of which inform the creation of systems and policies that determine or influence health. This recognition fundamentally problematizes over-reliance upon methodologies that cannot accommodate or interrogate these dynamics.

\subsection{Photovoice as a Foundation for Methodological Innovation}

As noted in Chapter One, the photovoice method, a form of participatory action research (PAR), offers a potential exception in public health to the epistemological and methodological constraints that have persisted in traditional and even PAR approaches. Photovoice not only invites participants to be fellow researchers, but it also expands: methods of communication, by using photos and imagery; means of elicitation, by taking 
a programmatic approach and using photo-documentation; and mechanisms both for data dissemination and community involvement, by culminating in public exhibits. The growing uptake of photovoice in public health research (illustrated in Figure 2.1), along with the method's unusual exploration of image-based communication, indicates the method's ability to extend public health practices. This uptake also renders photovoice a significant starting point for further methodological innovation—-particularly related to trauma-informed, culturally responsive inquiry and the potential value of arts-based methods. The following section begins that innovation process, providing some background on the photovoice method, notable critiques, and a discussion of the method's untapped and under-researched potential.

\subsubsection{Background}

Photovoice was developed by Carolyn Wang and Mary Ann Burris in the 1990s as a means of improving research among vulnerable and marginalized communities. Grounded in critical theory (described in the Introduction), Wang and Burris's method responded to the recognition that marginalized individuals are the experts in their own life experiences (Collins, 2000), and that they must be given opportunities to speak to and about decisions that affect them. The new method was also informed by documentary photography as well as by Paulo Freire's (2005) argument that visual images of daily experiences can raise critical consciousness by stimulating active reflection and dialogue about the power structures and relational dynamics that (over-)determine our circumstances. Wang and Burris described photovoice as "a process by which people can identify, represent, and enhance their community through a specific photographic technique" (Wang \& Burris, 1997). 
Over the last two decades, fields including public health, health education, sociology, social work, psychology, education, and more have utilized photovoice in research designs, indicating that its "photographic technique" is far from "specific." Researcher approaches have varied widely as they have tested the effectiveness of photovoice among such populations as rural village women in China (Wang, 1999), urban youth in the U.S. (Delgado, 2015); sex workers (Desyllus, 2014); homeless youth (Bender et al, 2017); men and women with HIV (Teti et al., 2016); citizens of KwaZuluNatal (a small rural town in South Africa) (Harley, 2012); and many others. Yet at its most basic, photovoice is a process in which participants are asked to take pictures that depict their lived experience. They then gather together to share and discuss their photographs: a group component designed to foster critical consciousness and stimulate ideas for community action that leads to change. Wang and Burris (1997) described the purpose of photovoice as threefold: "(1) to enable people to record and reflect their community's strengths and concerns, (2) to promote critical dialogue and knowledge about important issues through large and small group discussion of photographs, and (3) to reach policymakers" (p. 369).

\subsubsection{Popularity}

As previously noted, the popularity of photovoice has risen exponentially in several disciplines, including in health-related fields (see Figure 2.1). This is in part because its focus on policy influence appeals to public health practitioners committed to addressing the social determinants of health and health in all policies. Similarly, its unique mechanisms for expanding expressive and representational options are increasingly useful as the field seeks to better include historically underrepresented 
voices in research, depictions, and priorities related to community issues and resources (O’Mara-Eves et al., 2015; Poletta et al., 2011; Morgan \& Lifshay, 2006). Due to these advantages, photovoice looms in public health as a go-to creative strategy for traumainformed and culturally-responsive participatory action research. ${ }^{36}$ At present, it is also the only commonly taught and utilized research method in public health that builds upon creative practice - imagery, metaphor, metonymy - to generate data and stimulate action. These unique affordances and the method's rising popularity provide a critical foundation for the development of methodologies that support trauma- and culturally-responsive inquiry while responding to multiple levels of the social ecological framework.

\footnotetext{
${ }^{36}$ Note that photovoice is not always or even frequently cited as offering a "trauma-responsive research method;" rather, it is often described as being ideal for working with marginalized and vulnerable populations. This alternative description indicates researchers' recognition that sensitivity, creativity, and expressive options are needed when developing knowledge with and among such populations - and the ability of photovoice to meet these needs. Researchers may not yet readily recognize the consideration (and meeting) of these needs is part of taking a trauma-informed approach.
} 


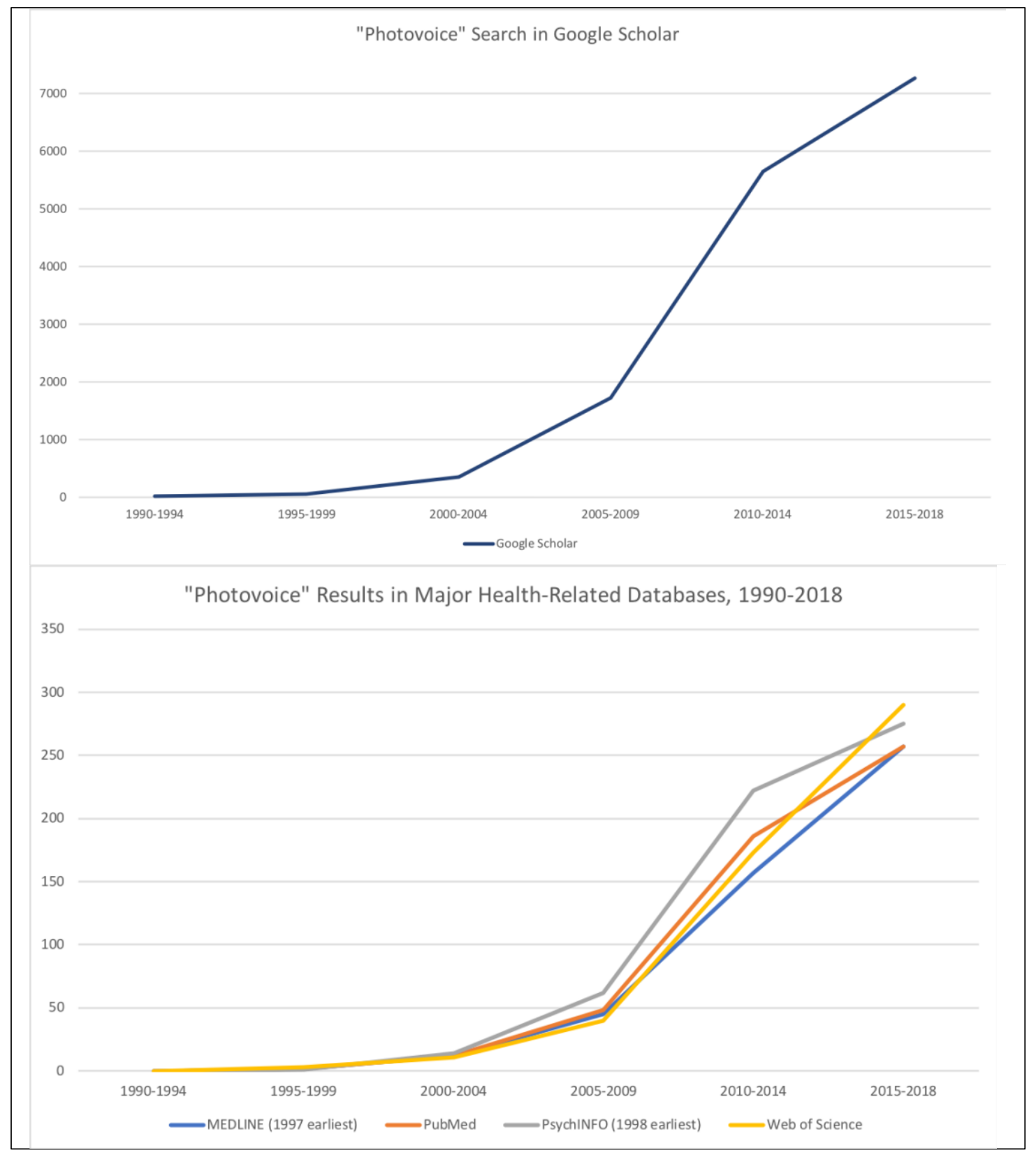

Figure 2.1: Popularity of photovoice over time

\subsubsection{Four Limitations}

Despite its benefits, accumulating photovoice studies have indicated consistent limitations that must be considered if researchers are to utilize and build upon photovoice approaches. These include failures to center communities, copyright and ownership 
conundrums, problematic empowerment rhetoric, and broken promises related to policy influence. ${ }^{37}$ By identifying the method's limitations, the present work can hypothesize methodological paths toward increased effectiveness and informed innovation.

\subsubsection{Community Centered?}

A primary allure of photovoice, as with PAR generally, has been its intention to include communities in research about them and to help effect real change on the communities' terms. However, the literature reveals that photovoice projects often fail to include participants beyond data collection—with researchers conducting subsequent coding, analysis, and publication either alone or with fellow academics (Evans-Agnew \& Rosemberg, 2016). More problematically, many academic publications have focused less on community-centered outcomes than on what a given photovoice project reveals about the method's effectiveness for that population (Delgado, 2015; Desyllas, 2014; Bender et al., 2017; Teti et al., 2016; Strack, Magill, \& McDonagh, 2004). Such information is relevant almost exclusively to fellow researchers. Other publications focus on the new "insider" knowledge that the method generates for outsiders (Desyllas, 2014), rather than on new knowledge that benefits participants and community members themselves. ${ }^{38}$ In short, researcher-initiated goals and deliverables are explicit and commonplace in the literature and, to the extent that researchers simultaneously describe photovoice as a community-centered method, the contradiction is problematic. As public health seeks to

\footnotetext{
${ }^{37}$ For more on ethical concerns related to photovoice research, see Evans-Agnew \& Rosemburg, 2016; Johnston, 2016; Sanon, Evans-Agnew, \& Boutain, 2014; and Shankar, 2016.

${ }^{38}$ These issues are not inherently problematic; after all, photovoice still often outperforms traditional research methods in its ability to generate feelings of connection and accomplishment among participants (see "Direct Benefits," below). In addition, photovoice authors may simply share different information with community members or the media, reserving analyses of the method and of the insider knowledge it generates for academic journals. (Certainly, scholarly articles are not ideal sites for the delivery of information to community members.) But when photovoice is hailed as community-centered while centering researchers' goals, a change in rhetoric (if not in practice) is clearly required.
} 
increase health equity, generate trauma- and culturally-responsive methods, and improve mechanisms of representation, it must build greater transparency and accuracy of description, practice, and dissemination into its (new) approaches.

\subsubsection{Ownership and Copyright Concerns}

A second limitation of photovoice relates to issues of ownership, copyright, and consent that have frequently come up during photovoice projects - such as researchers keeping photographs taken by participants, or failing to share decisions regarding whether exhibited photographs are sold. ${ }^{39}$ Unlike interview transcripts and notes, which scholars can typically expect to keep for their own purposes, photographs are often meaningful artifacts that warrant explicit ownership considerations on behalf of their creators. ${ }^{40}$ Importantly, ownership dilemmas are not a limitation of the photovoice method so much as evidence of its unique potential. That photovoice occasions the creation of art that its participants may find meaningful and compelling — and therefore worthy of keeping - is one of its many strengths. Unfortunately, many scholars have focused on photovoice as a research method to the neglect of recognizing it as an arts-

\footnotetext{
${ }^{39}$ Bender et al. (2017) describe a photovoice exhibit scenario in which community members asked researchers if they could purchase some of the participants' photographs. Caught off guard, the researchers suddenly felt they were "balancing our interest in having young people make decisions about their own work" with the need to "protect the intention of the project" (p. 387). In this instance, project leaders' failure to: 1) transparently discuss who owned the photographs, 2) negotiate what would happen to those photographs post-project, and 3) prepare for requests for purchase (a common scenario in exhibit settings), prevented them from including participants in decisions about their own intellectual and physical property. This is alarming for anyone pursuing ethical research, particularly when working among vulnerable and underrepresented populations.

${ }^{40}$ The use of artistic methods in research, including photography, "highlight[s] the need to rethink traditional research roles as it is no longer appropriate for researchers to maintain control over data that are collaboratively generated or for research participants to provide consent in advance of actually experiencing what is involved in using the arts to document their health and/or illness experience" (Cox \& Boydell, 2015).
} 
based intervention, preventing them from responding to photovoice artifacts as artistic creations. ${ }^{41}$

\subsubsection{Problematic Empowerment Rhetoric}

A third limitation of photovoice is found in the frequent use of empowerment rhetoric in the photovoice literature. Although empowerment is not one of Wang and Burris's (1997) original goals, photovoice study authors routinely note that participants experience "empowerment" as a result of exploring their lives and stories via photovoice (Delgado, 2015; Bender et al., 2017; Desyllas, 2014, etc.). While the term is consistent with the method's Freirian roots, when used uncritically or without an explicit definition, it risks misrepresenting or obscuring participants' actual experiences by presuming to name or categorize them. Quick employment of the term may also curtail interest in studying or elaborating upon photovoice's direct or immediate health benefits, ${ }^{42}$ instead haphazardly grouping such benefits together under an ambiguous label.

Use of empowerment rhetoric may also misrepresent the ability of photovoice to engender, direct, or manifest power. Mary E. Buckley (2000) argues that there is "nothing inherently empowering about participatory methodologies" (p. 44), and that "empowerment must be defined and contextualized in a manner which is directly relevant to the beneficiaries themselves" (p. 47). While researchers (or their methods) may indeed elicit stories, enable and support participants, amplify voices, and disseminate/circulate ideas, the extent to which these actions are "empowering" is both subjective and highly dependent upon how the term is defined. In addition, many photovoice study authors

\footnotetext{
41 Ownership and consent concerns in photovoice studies have been addressed in-depth by Pain, 2012; Johnston, 2016; and Harley, 2012.

${ }^{42}$ Potential outcomes, discussed in the following section, could range from social connection and selfesteem to the various health benefits associated with disclosure.
} 
have themselves been unable to access sociopolitical power, such as by connecting with policymakers. This suggests that they should be hesitant to view themselves or their methods as capable of "empowering" participants — at least without explicitly defining the term.

As an final empowerment-related concern, "empowered" seems often to be wielded as a synonym for participants' stated appreciation for having been able to express themselves and share their story. But this is not only inaccurate (telling one's story is not "axiomatically empowering" (Golden, 2017, p. 178)), but using "empowerment" in such cases suggests that, by offering opportunities for articulation and expression, photovoice proffered power that participants had not already possessed. While this may at times or in some sense be true, assuming its truth risks reinforcing a view of participants as powerless, uncommunicative others in need of saving. It also risks portraying photovoice as in some way salvific.

\subsubsection{Broken Promises Regarding Policy Influence}

Finally, and perhaps most problematically for those interested in health equity and health in all policies, photovoice projects have often promised political influence while routinely failing to deliver (Shankar, 2016; Johnston, 2016). Most photovoice publications list "reach[ing] policy makers" as a primary goal of the project (Wang \& Burris, 1997, p. 369); however, very few report having actually influenced policy or policy makers, with some authors noting their inability to make contact with policy makers at all (Desyllus, 2014; Strack, Magill, \& McDonagh, 2014). Catalani and Minkler (2010) observe in their literature review that "none of the photovoice articles [they] reviewed adequately discussed or evaluated the impacts of photovoice, if any, on the 
policy level” (p. 445, emphasis mine). Similarly, Evans-Agnew and Rosemberg (2016) observed that "many [photovoice] research designs are lacking in rigor to successfully harness" the opportunity photovoice presents for "mitigat[ing] and transform[ing] social injustice" (p. 1028).

To be fair, it is generally "difficult to attribute policy change to a single program" such as a photovoice project (Strack et al., 2010, p. 635), and changes in physical and social environments often require long-term effort. ${ }^{43}$ And notably, this difficulty is sustained by structural constraints such as funding determinations, evidence-based requirements, and publication demands - all of which often limit the types of accepted or measured outcomes, and rarely allow for long-term inquiry. Nevertheless, when policy influence is repeatedly noted in the literature as a primary goal of photovoice, the method's consistent inability to meet that goal becomes ethically problematic. ${ }^{44}$ The benefits of photovoice as a participatory, anti-oppressive research strategy are undermined when researchers are able to publish about photovoice projects - thereby gaining personal career "wins"-_regardless of whether participants realize any of the sociopolitical influence they had been led to anticipate. ${ }^{45}$ If the field is to advance equity, inclusion, and representation, researchers must choose and explain methods with realism, transparency, and accuracy. ${ }^{46}$

\footnotetext{
${ }^{43}$ See also Black's (2002) argument regarding the limited degree to which research in general has effected (or even informed) policy change.

${ }^{44}$ These observations are not meant to suggest intentional deception on the part of photovoice researchers; many may simply be overly optimistic, or new researchers may misconstrue the method itself as being capable of delivering results on its own.

${ }^{45}$ As a partial solution, Gloria Johnston (2016) recommended that photovoice be understood as "policy informing rather than policy changing" (p. 807; italics in original). Yet given that photovoice researchers are often unable even to make contact with policymakers, this more limited characterization still warrants caution.

${ }^{46}$ Notably, the accumulation of photovoice studies over two decades does reveal what the method most consistently delivers: new knowledge about how photovoice "works" with a given population, enhanced
} 


\subsection{Beyond Photovoice}

Critical reflection on the above limitations of photovoice generates a foundation from which photovoice can inform new trauma-informed, culturally-responsive methods of inquiry in public health. This section draws upon critical theory, critical narrative and expressive writing, and arts-in-health research to begin building upon photovoice's limitations. It approaches this process in three steps: first, it presents photovoice as an arts-based method, and thus as a means of opening up similar strategies; second, it denotes benefits and applications of photovoice that have yet to be explored or prioritized in public health; and third, it considers experimental strategies that are grounded in the current project's theoretical frameworks, which will be analyzed and discussed in subsequent chapters.

\subsubsection{Photovoice as an Arts-Based Method}

\subsubsection{1 "Art" in the Literature?}

Curiously, the originators of photovoice did not present it as an arts-based research method. Wang and Burris's publications $(1997,1998,1999)$ referred to community culture and imagination, inspiration, drawings, the importance of storytelling (including the importance of telling stories that go beyond words (1997, p. 372)), documentary photography, vision, voice, narration, lore, emotional power, scrapbooks and albums, rich colors and hues, creativity, creative expression, and ingenuity. However, they do not employ the word "art." The women who participated in their initial photovoice project in China were esteemed by study authors for being knowledgeable

understanding of a given population among its outsiders, and an engaging experience for participants/insiders (Catalani \& Minkler, 2010; Pain, 2012). These are worthy results, but they do not necessarily align with the frequently-mentioned photovoice goals of centering community objectives and priorities. 
narrators, creators of powerful images, critical thinkers regarding images, and more; yet they were never referred to as "artists" or creators of art. In fact, Yunnan participants often gifted the photographs they had taken to their family and friends, and Wang and Burris (1998) explicitly likened this action to an artist's gifting of a painting (p. 83). Yet they refrained from referring to the photographs themselves as art forms being gifted by artists. Similarly, Catalani and Minkler's (2010) extensive, oft-cited photovoice literature review does not mention the word "art" at all, except to state the need for a review of "the state of the art" of photovoice (p. 425). Perhaps both as a result and ongoing cause of art's absence in the literature, the fields of public health and health promotion have been hesitant or unable to recognize photovoice as arts-based, or to research and deploy other arts-based research $(\mathrm{ABR})$ methods.

Meanwhile, related fields_-including social work (Desyllus, 2014), nursing (Coad, 2007), sociology (Tarr, Gonzalez-Polledo, \& Cornish, 2018), education and youth development (Delgado, 2015; Leavy, 2015), and health psychology (Camic, 2008)—have identified photovoice as one among many arts-based methods. This contextualization of the method serves as a reminder that the successes of photovoice projects are due not to a magic specific to photography or to the photovoice process, but to the method's ability to initiate creativelystrategic means of expression within a shared framework that encourages safe and mutual exploration, personal storytelling, and critical thinking. Ideally, the method also generates mechanisms for amplification and dissemination that can increase participants' local influence and potentially impact policy. These multiple benefits are available via many other creative processes and interventions. ${ }^{47}$ When public health researchers recognize photovoice as a single

\footnotetext{
${ }^{47}$ Putland (2008) argued that "photographic documentation makes creative use of the enduring science/art tensions between photography as art form and research tool, drawing on its symbolic and iconic qualities
} 
application of usefully creative, communicative mechanisms, they will become better equipped to consider whether forms of art other than photography may be better suited to a given population, issue, or objective.

\subsubsection{Community Art is Data}

The recognition of photovoice as arts-based invites increased researcher acknowledgment of participant communities as spaces in which (some form of) art was likely being created long before any researchers arrived. For example, explicitly recognizing picture-taking as a form of creative expression may lead public health researchers to practice intentional inquiry regarding forms of creative expression that already exist in a community — and what these could reveal about that community's needs, assets, priorities, and more. Indeed, the collection and analysis of existing art from within a target population is a largely unexplored frontier within public health research. ${ }^{48}$ Yet art regularly happens without researcher initiation, and a quickness to initiate a photography (or other arts-based) project risks minimizing existing artistic assets while unnecessarily (re)centering researcher goals and timelines.

This is not to suggest that all members of every community have access to artistic practices; on the contrary, a researcher's intervention/initiation may often provide opportunities that did not exist before. However, public health researchers and

and potential to communicate in non-rational, non-literal ways (p. 273). Importantly, other forms of art offer the same opportunities for creativity, tension, symbolism, and non-rational, non-literal communication.

${ }^{48}$ Of course, access to means of art-making and cultural production — as well as time, space, and freedom to create-vary widely across populations, geographies, socioeconomic status, et cetera. However, increases in access to cameras (via phones), as well as increased access to opportunities for the digital dissemination of photographs, suggest the utility in public health of searching, collecting, and analyzing existing local/population photographs when conducting needs assessments. Other cultural-technological shiftsincreased means of making and sharing videos, recording music, podcasting, et cetera - suggest similar value in pursuing data collection methods that utilize existing creative communications from within the target population. 
practitioners should never assume that their intervention is the first or only opportunity a community or population has had (or taken) to articulate their lived experience.

\subsubsection{Structural Constraints (Again)}

While some researchers may simply have never thought of photovoice as "artsbased," it is also clear that there exist potential drawbacks to claiming the arts label in public health. As argued above, public health regularly functions from an objectivist epistemology that assumes quantitative methods provide superior data (Ashcroft \& Katwyk, 2016). As a result, scholars who do any form of qualitative research may find themselves defending their work against accusations of subjectivity, value-laden interpretation (Padgett, 2009), or scientific illegitimacy. Researchers may therefore see the label "arts-based" as inviting even greater skepticism.

However, as already argued, a social ecological approach invites the recognition that traditional forms of inquiry are themselves influenced by narratives and power structures laden with values and assumptions (see House, 1980, p. 41; Barad, 2007, p. 49). And as Eric Oberheim warned, "[ $\mathrm{t}] \mathrm{o}$ be convinced that one perspective delivers the truth is to be blinded to the truths offered by others" (Feyeraband, 2011, p. x). Using multiple methods allows scientists to better "face up" to "the limited, partial, and situated" nature of research practices and findings (Shotter, 2012, p. 321); and this is essential if we are to meaningfully include diverse cultures and knowledges, ${ }^{49}$ increase reciprocity and access to participation, and construct equitable, effective, and sustainable public health responses.

\footnotetext{
${ }^{49}$ For more on the value on cultural relevance/competence, see CDC, 2014 and Fleckman et al., 2015.
} 
Importantly, photovoice has established its ability to illuminate previouslyunrecognized perspectives using a culturally-sensitive approach. And given the growing popularity of photovoice and the field's uptake of a social ecological perspective, public health may actually be at or near a methodological tipping point. Claiming the artistic basis of photovoice may no longer jeopardize the method's perceived credibility so much as help the field acknowledge important affordances of creative strategies.

\subsubsection{Limited Exposure and Training}

Finally, researchers in public health may fail to recognize photovoice as artsbased simply because their formal training did not alert them to ABR as a methodological category. This is worth noting, as arts-based research strategies have long been utilized in fields such as education, social work, psychology, nursing, and sociology (Leavy, 2015), with methods ranging from narrative inquiry and life writing to poetry, music, theatre, dance, and the visual arts. Like photovoice, these approaches can help decenter researchers; illuminate information that otherwise fails to surface; provide new or safer means by which participants can tell their stories; create intuitive opportunities for public education or involvement; maintain the interest of participants; capitalize on the emotional power of art; stimulate civic action; and of course, reach and inform policy makers. Therefore, to the extent that public health education and practice overlooks or discounts arts-based methods, the field limits its ability to recharge its communications practices, enrich its data, and improve its cultural and intersectional responsiveness. The

potential for public health to overcome this limitation motivates the following discussion. 


\subsection{Untapped Potential, Implications, and Exploration}

The arts-based nature of photovoice, in conjunction with research regarding critical narrative, expressive writing, and arts in health and health care, helps reveal benefits of photovoice beyond those prioritized by its founders and by the majority of photovoice publications. These benefits include improved disclosure and (therefore) improved data collection; direct health benefits; and improvements to publicity and advocacy ${ }^{50}$ Considering these opportunities is vital to the current project, because it speaks to trauma-responsiveness, cultural relevance, and the overarching need to engage multiple levels of the social ecological model. This section therefore reviews four unique and relatively unexamined affordances of photovoice in public health, each of which will be used to open up the role of the arts-based methods in public health more generally.

\subsubsection{Aiding Disclosure, Improving Data}

Most immediate among photovoice's unsung benefits may be its ability to aid disclosure and discussion, thereby expanding the topics and issues about which researchers and practitioners are able to learn. For example, in a photovoice study among homeless youth, participants described being able to "share experiences which would otherwise be difficult to express and process" (Bender et al., 2017, p. 383). Similarly, Teti et al.'s (2016) study among women and men with HIV revealed that "[p]hotovoice...provided a way for reluctant participants to strategize to engage in different, safer or more positive disclosures" (p. 47). ${ }^{51}$

\footnotetext{
${ }^{50}$ While some of these benefits have been mentioned in the literature, they have yet to be fully theorized, tested, or expanded in public health.

${ }^{51}$ Additional studies that in some way note the photovoice process as having aided the disclosure or discussion of sensitive topics include Desyllas, 2014; Shankar, 2016; and Simmonds et al., 2015, among others.
} 
It is likely that the arts-based nature of photovoice contributes to such increases in safety and disclosure. By using photography, participants can create personal distance (if they wish) from the issues or concerns they are examining, which can aid both communication and critical thinking (Nicholls, 2009). Photovoice also allows the use of image and symbol, which can aid communication when putting one's experience into words is too challenging. ${ }^{52}$ Importantly, the use of photography may also increase accessibility overall.

These effects have significant implications for public health research and practice, which can be revealed through several questions: What do we not know about a given population because individuals are unable to reveal a particular experience or vulnerability? What do we miss when surveys or interviews are textocentric, demand forthright disclosure, or define experiences differently than participants? And how might failures to gather more complete knowledge be affecting funding and resource decisions, health care access and delivery, and more? Such questions are critical given the ubiquity of adverse childhood experiences (ACEs) and individual and community traumas. To prevent harm, obtain the most complete information, and improve health services overall, researchers must explore a variety of data collection methods by which individuals and communities can safely and productively tell their stories.

\subsubsection{Direct Health Benefits}

Although public health research is typically conducted with the goal of informing or developing benefits rather than directly providing them, evidence suggests that research can be structured to offer direct benefits. For example, as revealed in hundreds

\footnotetext{
${ }^{52}$ See van der Kolk (2015) for more on the difficulties of verbalizing traumatic experiences.
} 
of expressive writing studies, simply writing or narrating difficult experiences results in benefits among study subjects such as improved immune function, fewer sick days, and improved grades (Frattaroli, 2006; Pennebaker, 1999; Travagin et al., 2015). Such results could also follow photovoice participation (depending upon how it is structured), given that photovoice frequently involves discussing or writing about difficult histories, contexts, and experiences.

Similarly, putting one's experience into a cohesive narrative has been shown to cultivate a greater sense of meaning and control (Goodson \& Gill, 2014; Greenbaum \& Javdani, 2016; Chandler et al., 2015), and the development of critical consciousness can generate increased self-efficacy and self-determination (Schwarzer \& Warner, 2013; Bernard, 1995; Rimer, 2017). These outcomes are all linked to increased resilience - the ability to recover and thrive despite challenging conditions ${ }^{53}$ - and these activities are common aspects of the photovoice process.

Finally, feeling heard and respected when sharing one's story can reduce perceived isolation, challenge stigma, and improve self-esteem and self-determination (Alvarez \& Mearns, 2012)_factors that have been linked to increased well-being (Seppala, Rossomando, \& Doty, 2013; Link \& Phelan, 2006; Fox, 2012; Edge, Newbold, \& McKeary, 2014). Photovoice frequently offers opportunities for this type of sharing in the form of group conversations or public exhibits where participants' work is seen and discussed.

To be sure, the actual realization of such benefits in a photovoice study relies heavily on the population, facilitators, program length and structure, objectives, and

\footnotetext{
${ }^{53}$ Resilience is essential for long-term health, as it helps mitigate the effects of trauma, stress, and other difficult circumstances (see Aburn, Gott, \& Hoare, 2016).
} 
many other variables. However, their potential deserves greater exploration, as well as a more prominent role in photovoice planning, research, and publication. ${ }^{54}$ By aiming for direct health benefits, researchers can productively reframe their goals, approaches, and ethics to consider not only harm avoidance but the capacity to improve health while researching it. ${ }^{55}$ Examining potential health benefits involves prioritizing or enhancing aspects of the research process that facilitate direct improvements-such as disclosure, group connection, narration, feeling seen, etc.

\subsubsection{Framing and Storytelling}

Another relatively unsung benefit of photovoice is its ability to support the framing and storytelling required to advance policy changes and community issues. For example, the literature has noted photovoice's ability to humanize participants and issues (Wang et al., 1998, p. 81; Wang et al., 2004, p. 913) and to increase the persuasiveness of participants' arguments (Wang et al, 1998; Desyllus, 2014). Exhibited photographs, in combination with participant narratives, offer a new "frames" through which audiences can better empathize and understand the experience of an other-often creating memorable shifts in perspective. ${ }^{56}$

Arts-based methods also aid framing and storytelling by creating intuitive occasions for press coverage — which can help disseminate public health messages, shift public opinion, and potentially generate political will. Photovoice exhibits offer press outlets local human interest events that involve and appeal to the public, and positive

\footnotetext{
${ }^{54}$ Notably, published photovoice studies do frequently mention positive participant responses (Desyllus, 2014; Bender et al., 2017; Mayfield-Johnson, 2017; Catalani \& Minkler, 2010); however, as argued earlier, these are often labeled as participant "empowerment" rather than intentionally discussed or measured as outcomes.

${ }^{55}$ This process would also increase research reciprocity.

${ }^{56}$ See Lakoff, 2008 for more on the importance of metaphor and framing in message uptake.
} 
media responses are apparent in the literature ${ }^{57}$ Public health would therefore do well to explore additional ways in which arts-based methods and deliverables could support broad awareness, engagement, dissemination, and advocacy efforts. Given that the field has recognized the need for innovative methods of outreach, public education, and policy advocacy (Brownell, Price \& Steinman, 2013; Bernhardt, 2010), photovoice's arts-based, public-interest successes offer important insights.

\subsection{Next Steps: Building on Photovoice and Theoretical Frameworks}

As we have seen, photovoice offers unique affordances in public health research. It is known for keeping participants engaged, increasing empathy, and creating opportunities for self-representation. We have seen that the photovoice process can aid disclosure and increase accessibility, thereby improving data; it may also offer direct health benefits, increase public interest, and generate new opportunities for press and circulation. Importantly, these benefits can be traced to the method's grounding in artistic or creative practices - which are not exclusive to photovoice. And because the photovoice process has often offered a trauma-informed and culturally-responsive approach, it serves as a starting point for improved methods of inquiry in the field. This section draws upon these concepts to present four considerations regarding the role of the arts in trauma-informed, culturally-responsive inquiry in public health. These considerations have informed the design of the methodological prototypes described and tested in Chapter Three.

\subsubsection{Trauma-informed practice requires expressive options.}

\footnotetext{
${ }^{57}$ Photovoice projects that were unable to reach policymakers were often nevertheless able to generate media interest in their exhibit and other artifacts (see Desyllus, 2014).
} 
As noted in Chapter One, the equitable inclusion of diverse voices in public health research demands that researchers accommodate individuals whose experiences of trauma or stigma render their participation in traditional studies, formats, and venues unsafe or inaccessible (Baker et al., 2016; SAMHSA, 2014; Bloom, 2018). And as noted earlier in this chapter, we must be willing to ask what we miss about a given population when individuals are unable to reveal a particular experience or vulnerability (Napier et al., 2014). We must also consider that textocentric methods (including interviews and focus groups) and predetermined definitions or labels may exclude individuals and experiences that should inform our work. As mentioned above, photovoice studies have shown that the opportunity to express one's beliefs, values, or experiences through an alternative medium can enable disclosure - making such alternatives essential to public health research. When one considers that trauma is "disproportionately experienced by certain groups because of deeply entrenched structural inequalities" (Bowen \& Murshid, 2016, p. 223), it is clear that failures to improve trauma-informed practice by expanding expressive options could perpetuate ongoing health inequities. Fortunately, as suggested by photovoice, there are many creative means by which humans can communicate beliefs, values, needs, experiences, and assets. With continued methodological innovation, as well as training and education, the field can expand communicative options for researchers, study participants, and target populations.

\subsubsection{Move beyond photography.}

As suggested throughout this chapter, there is no clear reason for public health researchers and practitioners to stop at photography when searching out expressive options; nor is there reason to limit photovoice itself to the few primary goals listed by 
the method's founders. This is particularly true given the described potential for artsbased methods to improve data, support trauma- and culturally-responsive practice, and offer direct health benefits. The success of the photovoice method lies in its capacity to offer creative strategizing, increased nuance of feedback, narrative choice, participant engagement, safer disclosure, cultural adaptability, self-representation, empathy and connection, community influence, press potential, and so on. Notably, these benefits are accessible via many forms, from writing poetry, screenplays, and memoirs to painting, dancing, acting, or composing music.

\subsubsection{Cultural responsiveness requires exploration.}

While it may seem that photography is more intuitive or accessible than some of these additional art forms, no single form of creative expression will be ideal for every population, individual, location, research question, or study objective. Public health researchers must therefore be open to engaging the forms of expression and communication that most resonate with communities, or that will most effectively persuade targeted outsiders regarding a given population's requests for action.

Researchers must also be open to the innovation of analytical methods designed for these new communications. The key to choosing an art form (or several) may not be participants' assumed experience or ease with a given activity, such as taking photos, but rather context, culture, interest, framing potential, and purpose. ${ }^{58}$

\subsubsection{Look for the art already being made.}

As argued above, the successes of photovoice should alert researchers to the potential value of art that is already being created in a given community. Access to art, as

\footnotetext{
${ }^{58}$ In keeping with the Freirian power-sharing at the center of the original photovoice methodology, decisions regarding expressive forms may be reached in partnership with the community.
} 
well as its production and valuation, differ greatly among communities and their individual members; nevertheless, honored art practices in any community — a wellattended poetry slam; a local album everyone talks about; a theatrical performance that sells out — can offer community health insights as powerful as those offered by photovoice. If art can inform public health practice, this is true whether the art was directly elicited for the purposes of research or not. This understanding has significant implications for future public health research and practice.

\subsection{Conclusion}

This chapter discussed the incomplete shift in public health from a biomedical to a social ecological paradigm of health, describing the need for methods that can draw knowledge from diverse sources and practices, thus improving public health's relationship with and understanding of communities and populations — including their health, needs, assets, values, and priorities. It then discussed photovoice as a method that challenged traditional understandings of the following: communication and data, by utilizing photographs; evidence, by relying on image and narrative; levels of change, by emphasizing policy; and even reality itself, by highlighting the role of perception and story in how a community is understood, depicted, or resourced. After identifying four limitations of photovoice, this chapter discussed several benefits of the photovoice method that deserve further exploration, and noted the ability of other art forms to produce similar, perhaps even greater, benefits. This was followed by four considerations for the incorporation of more art forms in public health research, improving our capacity for traumaand culturally-responsive inquiry.

The current study now draws on these considerations to create three prototypes for methodological innovation. These move beyond photography to test creative writing and theatre 
as methods of inquiry among three population samples in a southern U.S. city. In addition to testing non-photographic art forms as sources of data, they also test three distinct methods of collecting these data. The following chapter offers details regarding these prototyped methods themselves, as well as the research populations, research questions, and data collection processes involved. 


\section{CHAPTER THREE: METHODS}

Grounded in the preceding discussions regarding trauma-informed, culturallyresponsive inquiry, photovoice strengths and limitations, and opportunities for innovation, this study compared data gathered via conventional methods (quantitative surveys) to those gathered via three prototypes of arts-based methods. This process modeled Chapter Two's recommended move beyond photography by analyzing creative writing and theatrical performance as arts-based sources and venues for public health research and practice.

\subsection{Design Rationale}

For the purposes of the current study, all included methods needed to be compared for their strengths and limitations in providing public health data; they also needed to be compared for their responsiveness to trauma, stigma, and cultural difference. To best make these comparisons, the prototyped methods had to be used together in research that was conducted: 1) among or about vulnerable or underrepresented populations, to illuminate cultural-responsiveness; and 2) in the context of stigmatized or potentially traumatizing topics, to illuminate capacity for traumainformed research that responds to the potential for stigma or oppression. In Louisville, Kentucky, gender disparities in local violence prevention efforts indicated a need for improved violence-related research among young women. This need presented an 
opportunity to make methodological comparisons while also conducting research that offered immediate benefits to the community. The current project is thus a nested study, functioning as a mixed methods study of violence among young women in Louisville, with the overarching goal of testing and comparing the utilized methods.

\subsection{Background of the Test Inquiry}

The city of Louis ville, Kentucky has made consistent efforts to reduce youth violence (CDC, 2016; Jones, 2017; Glowicki, 2014); however, consistent with much of the U.S., the city's prevention programs have often been "directed towards boys, and it is important to consider the uniqueness of girls by incorporating...efforts specifically designed for girls (Yonas et al., 2005). The World Health Organization (WHO) has noted that it is "important to look at gender as a determinant of adolescence health" when it comes to violence, and to find out "the main differences between girls and boys in exposure to risk, norms and values and access to services" (WHO Regional Office of Europe, 2011). Thus the researcher noted that, to reach its stated goals of decreasing violence and of increasing health equity (Center for Health Equity, 2018), Louisville would need to develop its awareness of ways in which young female-identified residents defined and perceived violence, the extent to which they experienced it, the multiple effects of those experiences, and ways in which women showed and built resilience.

\subsection{Population of Interest}

Therefore, the current study sought information from female-identified residents of Louisville, ages 11 to 21, regarding understandings and experiences of violence and safety. The choice of this population and inquiry satisfied the project's overarching goal of testing the ability of new arts-based methods to offer trauma-informed, culturally- 
responsive approaches to research inquiry. For example, because violence is fundamentally associated with trauma, ${ }^{59}$ violence-related research with young women must be trauma-informed. Similarly, the samples utilized in this study included vulnerable and underrepresented populations (youth, women, minority women), and they were composed of participants who did not necessarily share backgrounds, cultures, or preferred modes of expression. These factors indicated the importance of using culturally-responsive communicative options that could be adapted by each respondent.

\subsection{Sample Descriptions}

Given the natures of its inquiry and target population, this study used purposive and convenience sampling based on existing relationships. While this approach reduced the generalizability of the study's results, the study included three distinct samples, thus offering transferrable insights_-including themes, patterns, discrepancies, and recommendations for further research — from differing groups and contexts. This sampling approach was also strategic in that it allowed the examination of three distinct arts-based data sources and their effects. These sources were developed in response to considerations outlined in Chapter Two, and included:

- Creative writing elicited via direct intervention, which modeled a hands-on approach akin to many photovoice projects;

- Creative writing elicited via citywide calls for submissions, which modeled partnership with community arts organizations, and provided a comparison with broad survey invitations often used in quantitative approaches; and

\footnotetext{
${ }^{59}$ In fact, the terms "violence" and "trauma" often appear together, since violence is understood to be a primary cause of trauma. See SAMHSA, 2018; Magruder et al., 2016; Lynn-Whaley \& Sugarmann, 2017.
} 
- Unelicited theatrical writing and performance, which demonstrated the value of arts-based initiatives that originate within a population (apart from researcher intrusion).

The three population samples chosen for this study included: 1) girls who were incarcerated in Louisville's Youth Detention Services (YDS) in $2018(\mathrm{n}=27) ; 2)$ women ages 11 to 21 who resided in the metro Louisville area, and who entered a March 2018 writing contest ( $\mathrm{n}=94)$; and 3 ) female members of the University of Louisville's PEACC Center Survivor Network who had survived intimate partner violence, sexual assault, or related trauma, and had cowritten a play about their experiences $(n=5)$. Each of these populations were relevant to this study in that their insights and revelations contributed to the city's global understanding of violence among young women; however, each group was also included for distinct reasons. First, young women in the justice system are nearly twice as likely as young men to have five or more adverse childhood experiences (ACEs; Saar et al., 2015), and "the most common crimes for which girls are arrestedincluding running away, substance abuse, and truancy—are also the most common symptoms of abuse" (p. 9). In addition, incarcerated young women have been shown to have "significantly greater rates of physical, sexual, and emotional abuse and greater rates of physical neglect than boys" (Zahn et al., 2009, p. 267). Such findings suggest that young women in detention are disproportionately more likely to have witnessed or experienced violence and other difficult experiences; however, their needs often go unheeded in a system historically designed for males (Viar, 2017; Veysey, 2003; 
Sherman \& Balck, 2015). ${ }^{60}$ In other words, young justice-involved women remain underserved not only in local youth violence and safety efforts, but also in broad, national juvenile justice practices and reforms. As such, their inclusion in this study of violence among young women was strategic. In addition, given higher rates of trauma histories within this population, the testing of trauma-informed research practices, including artsbased methods, was critical.

The population of entrants in a 2018 writing contest were included in this study based on their ability to represent diverse, citywide conceptions and experiences related to violence. In 2018, the University of Louisville's Youth Violence Prevention Research Center (YVPRC) partnered with literary publisher Sarabande Books to host a writing contest during National Youth Violence Prevention Week (NYVPW) for young womenidentifying writers, ages 11 to 21 . The contest invited writers to share their "experiences with violence or peace, conflict or healing" (Stevens, 2018), the goal being to amplify young women's voices while helping the city of Louisville better incorporate women's perspectives, needs, and concerns into ongoing violence prevention work. Because the contest was widely promoted across various platforms, including youth arts organizations, the local school district, official city news feeds, and individual citizens' social media, its 107 submissions reflected diverse racial, ethnic, socioeconomic, and religious backgrounds, as well as varying ages, abilities, and literacy levels. This rendered the data informative, representing various experiences that contribute to public health knowledge and practice.

\footnotetext{
${ }^{60}$ In fact, the federal Office of Juvenile Justice and Delinquency Prevention (OJJDP) recognizes the issue of gender inequity in the juvenile justice system, and has strongly supported gender-responsive programs, policies, theories, and services (OJJDP, 2018).
} 
Finally, the inclusion of female members of the PEACC Center's Survivors Network ${ }^{61}$ who had written a play about their experiences of sexual assault was based on the immediate applicability of that play's subject matter to the study at hand. Co-written and performed in 2018, the play sold out its two performances, indicating that its content generated interest and resonance within the community. The script provided a significant source of arts-based data about sexual assault, and corroborated the argument from Chapter Two that such data often emerges in communities independent of public health research or intervention.

\subsection{Data Sources}

This study sought not only to investigate the above populations' encounters with and conceptualizations of violence, but primarily to compare the effectiveness of conventional and arts-based methods of inquiry and data collection in examining these factors. Therefore, analyses were conducted both of creative data sets and of quantitative survey results representing each of the three population samples. ${ }^{62}$

\subsubsection{Qualitative Data Sources}

Creative writings from girls incarcerated in YDS were drawn from a twiceweekly, writing-based health intervention that the researcher conducts within the detention facility. ${ }^{63}$ In this program, participants are regularly given creative prompts to which they can respond as they choose; when they write, they are asked if they would like their work to be considered for inclusion in books of poetry that are published by the

\footnotetext{
${ }^{61}$ The PEACC Center's focused purpose is to "provide advocacy and assistance to university students, staff and faculty who are affected by sexual misconduct, sexual violence, intimate partner violence and stalking" (PEACC , 2018). The writing of the play analyzed as part of this study-titled How to Start a Wildfirewas coordinated and overseen by PEACC Center staff and consultants.

${ }^{62}$ This study was approved by the University of Louisville's Institutional Review Board (IRB), study number 18.1016 .

63 This program is ongoing; it was not initiated for the purposes of the current study.
} 
researcher's program (Project Uncaged), in partnership with literary publisher Sarabande Books. All intervention facilitators emphasize to participants that they are under no pressure to share their writing, that privacy is a welcome choice, and that their decision either way has no bearing on their status in detention. If participants do choose to share their work, only pen names or initials are used to credit authors (to protect their privacy), and any names or identifying details within the content are redacted. Between January and September 2018, 85 separate pieces of writing were collected from 27 writers, totaling 6,829 words.

As noted above, creative writings from young Louisville women ages 11 to 21 were drawn from a citywide writing contest for young women, co-hosted by Sarabande Books and the researcher (through the university's YVPRC), in celebration of National Youth Violence Prevention Week in 2018. Entries were submitted via an online platform; in one case, a local English teacher emailed entries directly to the contest hosts, on behalf of students who lacked internet access. In total, 107 entries were received from 105 participants (one writer entered three poems). Of these, three entries were eliminated due to submission criteria (one was unviewable; one entrant identified as male; one entrant lived outside of Louisville); from the remaining 104 poems, 16 were selected for inclusion in an anthology titled, The Almost Mothers of the Greater Louisville Metropolitan Area, published by Sarabande Writing Labs in June 2018.

When Sarabande was presented with the possibility of including contest submissions in local research about violence, the organization contacted all online entrants to provide them with information and the opportunity to opt out; the study author directly contacted the teacher who had emailed entries. After a three-week opt-out period, 
one entrant had opted out, while 12 entrants remained unreachable. However, three of those 12 entrants had been published in Sarabande's 2018 anthology, rendering their work publicly available for analysis. In total, this process left 96 entrants (and 98 separate pieces of writing) for analysis, totaling 18,508 words.

Finally, creative writing from five students who are survivors of sexual assault, who were participating in PEACC Center programming at the University of Louisville, was drawn from a play called How to Start a Wildfire, which these survivors wrote and performed in January 2018. Though the play's two performances were quite public, the researcher obtained writers' permission ${ }^{64}$ to utilize the work in the current study, and to gain access to the script. This script provided a single piece of writing totaling 8,598 words.

\subsubsection{Quantitative Data Sources}

Only one of the three sample populations — girls in youth detention —include fully-corresponding survey data for direct comparison to their creative writing. For the other two samples (young Louis ville women who entered a writing contest, and female University of Louis ville students who wrote a play about violence), the researcher obtained survey data that is intensively reflective of the immediate broader population. Notably, given the circumstances and locations of both the writing and of the surveys' administration, the creative writers may indeed have also participated in the surveys whose data are utilized in this study.

Quantitative data for the first population sample, girls in Youth Detention Services, were drawn from surveys administered among 24 incarcerated girls in 2018 by

\footnotetext{
${ }^{64}$ Permission was obtained through the PEACC Center's director, who had coordinated the writing and performance of the play.
} 
the University of Louisville's Commonwealth Institute of Kentucky. These surveys were designed both to illuminate girls' health while in detention and to measure the effectiveness of the detention-based expressive writing intervention they were receiving. ${ }^{65}$ In addition to demographic information such as race/ethnicity, age, and sexual orientation, surveys included a modified adverse childhood experiences (ACEs) questionnaire, ${ }^{66}$ a modified version of the Child PTSD Symptom Scale (National Child Traumatic Stress Network, 2018), and an abbreviated version of the Devereux Adult Resilience Survey (Mackrain, 2013), which was modified for teen readers.

Quantitative data for the second population sample, young women living in the Louisville Metropolitan area, were drawn from an extensive survey administered in 2017 among students throughout Louisville's public school district (for females ages 11 to 21 , $\mathrm{n}=995$ ), by the University of Louisville's YVPRC. ${ }^{67}$ The survey utilized scales and metrics contained in the CDC's Measuring Violence-Related Attitudes, Behaviors, and Influences Among Youths: A Compendium of Assessment Tools (Dahlberg et al., 2005), and measured the extent to which respondents had experienced or witnessed violence, their personal engagement in and attitudes toward violence, and their perceptions regarding peer behaviors and attitudes related to violence. Demographic data were also collected, including race/ethnicity, gender, age, and grade.

Finally, quantitative data regarding University of Louisville females' experiences of violence, safety, and well-being were obtained from two University of Louisville

\footnotetext{
${ }^{65}$ In particular, this program was being evaluated for its ability to reduce participant stress or increase resilience.

${ }^{66}$ For more about the ACEs questionnaire, see Good Therapy, 2018.

${ }^{67}$ As a school-based survey, this data included young women ages 11-19, while the arts-based comparison data (a citywide writing contest) was open to women ages 11-21. The inclusion of different ages across the two studies will be noted in the analysis.
} 
surveys. While both surveys addressed health and safety, they collected different demographics, elicited different types of data, and were administered in different yearsrendering their data complementary. The first survey, called "CardSafe," was administered by the university's Office of Institutional Effectiveness in Spring 2016 (for undergraduate females, $n=376$ ). This survey collected demographic information such gender identity, sexual orientation, and school year (freshman, sophomore, junior, senior); it additionally measured respondent perceptions, experiences, and behaviors related to violence, safety, health, sex and relationships, and access to resources. The second, titled "Well-Being and Resilience," was administered by the university's Office of Health Promotion in Spring 2018 (for females aged 21 and under, $n=467$ ). This survey collected demographic information such race/ethnicity, gender identity, and age; it additionally measured respondent behaviors and beliefs related to health, well-being, safety, and access to resources.

\subsection{Plan for Analysis}

\subsubsection{Arts-Based Qualitative Inquiry}

The process of analyzing both the quantitative and qualitative data in this study was framed by the two primary research questions, which have been reformulated in this chapter to reflect the sample populations and the topic of violence. First, What does art by young Louisville women reveal about their experiences of violence; their associated needs and priorities; their values related to violence; and their assets in creating a safer, healthier city? And second, How do these data, including its collection and analysis, compare to those resulting from quantitative surveys? 
To answer the first question, analysis of creative texts from all three samples began with structural concepts coding using a broad frame of violence, and incorporating in vivo codes as relevant. As noted in previous chapters, a potentially significant affordance of arts-based platforms is that they can allow creators and participants to share more information than traditional methods. Investigating this affordance required that the process of analysis allow new themes or patterns to manifest. Thus, while the structural frame of violence was necessary given the research question, the process of structural/concepts coding simultaneously included open (initial) coding: recognizing the possibility that "violence" and related concepts could emerge in unusual and unexpected ways throughout girls' writing.

The first round of coding was conducted separately for each population; during the second and third rounds, codes were grouped into emerging themes—beginning within each set, and then across the three sets of writing. These continued to be regrouped and refined during the course of analysis. In addition, frequency counts were established for each population to help illuminate priorities and allow a narrowing of themes for discussion.

At this point, the researcher left qualitative analysis to conduct the majority of the survey data analysis (described below), later returning to arts-based data sets to begin assessing whether and to what extent survey data aligned with the themes and information resulting from the corresponding population's arts-based data. Finally, a similar process was employed in the opposite direction: identifying whether and to what extent the arts-based data elaborated upon, interrogated, or otherwise supplemented survey data. 


\subsubsection{Quantitative Inquiry}

Drawing on this study's research questions as well as on initial qualitative findings, survey data for each sample population were analyzed to identify (as possible): 1) study participants' experiences and definitions of violence or trauma; 2) indications of assets such as cultural identity, social supports, or other protective factors; and 3) beliefs, perceptions, or values related to violence. Beyond this broad approach, due to the fact that each of the three sample populations had taken different surveys, the analytical procedure differed according to survey and population.

For example, among the population in Youth Detention, surveys were administered the first time a new participant arrived in the writing workshop, and then weekly thereafter. Some participants attended several weeks, thus completing the survey multiple times, while others attended or took the survey only once. Thus to render data more comparable within itself and with other sets, analysis included data only from each participant's initial questionnaire. In addition, researchers had modified this survey's included instruments to accommodate the population served (incarcerated girls); these modifications required some adjustment and cleaning for the current study. For example, the survey separated one of the 10 classic ACEs questions into two parts, and added questions about experiences with homelessness, death of a loved one, or pregnancy. The researcher re-combined these two-part questions and separated out the others, rendering ACEs scores for each participant that align with national uses of this instrument. In addition, although the Devereux Resilience scale is typically not scored, this study coded "Not yet," "Sometimes" and "Always" in order to calculate levels of self-reported resilience among this population. Lastly, the Child Trauma Symptom Scale (CPSS) had 
been modified to ensure that respondents would have a shorter survey-completion time, and that they would not be asked to complete questions that were inapplicable in carceral settings. Thus out of 20 typical items on this instrument, the survey administered in YDS in 2018 included only eight. As a result, the highest possible score was 24 rather than 51, and scores could not be calculated as usual to determine the potential presence of PTSD. However, based on conventional cut-off ranges for PTSD severity (EPISCenter, n.d.), this study calculated the total trauma symptom scores from the items available, and then categorized results into the conventional ranges: "minimal" = 0-10; "mild" = 11-20; and "moderate" $=21-40$. This provided very provisional insights into levels of trauma symptoms among girls in YDS, at the time of their initial survey.

When analyzing survey data for Louisville females, the YVPRC's survey data had to be rendered more consistent with the population that had submitted creative texts to the writing contest. They were therefore disaggregated by gender, and limited to respondents within the age range of the writing contest participants (11 to 21). In addition, variables and survey constructs were limited to those that were relevant to the study at hand; these included: perceptions of respondents' community; sense of sociopolitical control (high school respondents only); exposure to violence; attitudes toward violence; cultural identity; and social supports. Once data were cleaned and prepared for analysis, new variables and descriptive statistics were calculated to identify trends and key findings.

When analyzing survey data for female students at the University of Louisville, the campus-wide "CardSafe Survey" data had to be disaggregated by gender; in addition, because information about age had not been collected, survey results were limited to students who identified as freshmen, sophomores, juniors or seniors. While this did not 
guarantee that results would strictly reflect respondents age 21 and under, it provided quantitative data comparable to the undergraduate student population which had produced the play script analyzed for this study. Similarly, the "Well-Being and Resilience" survey data were disaggregated by gender and limited to respondents aged 21 and under. For both surveys, results were narrowed to those variables and constructs consistent with the research questions, including: experiences of violence (victimization, witnessing); beliefs and knowledge about sexual assault, consent, rape, and similar; perceptions of safety/danger as a student of the University of Louisville; and behavioral responses to those perceptions.

For all three data sets, survey findings were compared to earlier analyses of creative writing from the same sample, with particular attention to whether and how the experiences or perceptions of violence and other factors aligned with, elaborated upon, or contradicted those indicated in creative writing. In addition, if survey findings led to new inquiries regarding the qualitative data, these were noted and incorporated into the third and final round of qualitative coding - completing the analysis for each sample.

\subsection{Population Comparisons}

In the final segment of the analytical process for violence-related data, all of the arts-based results and all of the quantitative results were analyzed for similarities, differences, and patterns among the three populations. The goal of this step was to identify violence-related experiences, perceptions, priorities, or other insights that were consistent across the distinct samples, and to highlight issues particular to a specific population. 


\subsection{Methodological Comparisons}

The above analyses comprised a mixed methods study of experiences and perceptions of violence among young women ages 11 to 21 in Louisville. Because the current project is ultimately designed to examine methodological prototypes for public health data collection, the final step in analysis involved observing initial affordances and limitations of each prototype, as compared to the quantitative surveys and to one another. This process is described and completed in Chapter Four.

\subsection{Limitations}

Each survey and each set of creative writing provided extensive data, the full analysis of which is beyond the scope of the current project. Given the nested nature of this study — a study of females' perceptions and experiences of violence within a study of the methods used - the focus was on comparing utilized methods and on determining the potential for data provision and triangulation, rather than on extracting as much information as possible from each data set. Because of this, important data regarding violence among Louisville females was inevitably excluded from this project. Opportunity therefore exists with all included data sets for additional research questions and analyses; in particular, gender comparisons within the latter two populations' quantitative data sets loom as important next steps in learning about gender disparities in experiences and perceptions of violence for the included populations.

In addition, the comparative process applied in this study is not without potential for bias or interpretive mistakes, particularly when analyzing creative writing. As noted in Chapter One, all researchers bring to analyses specific values and experiences; while this researcher's background in creative writing and rhetoric is likely to support rather 
than hinder effective analysis, it may also add its own biases. Importantly, this project is presented as the study of three prototypes; as such, it is not intended to fully explore experiences and perceptions of violence through a gendered lens, nor is it intended to undergird methodological overhauls in public health research. Instead, the study's findings will illuminate next steps for further prototyping, innovation, and reflexivity regarding research methods and their effects on health equity.

\subsection{Conclusion}

This chapter described the methods used in this study, including the choice of target population and study topic, population sample descriptions, data sources, processes of analysis, and limitations of the analytical procedure. The next chapter offers findings and initial interpretations of this data. 


\section{CHAPTER FOUR: ANALYSIS}

The purpose of this study was to test three methodological prototypes for data collection in public health, examining in particular their effectiveness in use with a vulnerable population regarding a potentially traumatizing topic. The study was designed to answer two research questions:

RQ1: What does art by young Louisville women reveal about their experiences of violence; their associated needs and priorities; their values related to violence; and their assets in creating a safer, healthier city?

RQ2: How do these data, including their collection and analysis, compare to data resulting from quantitative surveys?

Analysis involved examining creative writing from three sample populations, and comparing results to those of related quantitative surveys.

\subsection{Qualitative Process Overview}

As documented in Chapter Three, analysis of the arts-based qualitative data was conducted according to the sequence noted in Figure 4.1. At the end of all rounds of coding for all data sets, a total of 135 codes had been established. After establishing frequency counts for all codes, the researcher observed that codes for which there were fewer than 10 counts were often related to other more prominent codes, or they figured as

outliers across the data sets. Thus to further focus the study (and to illuminate population 
priorities), these codes were set aside for separate analysis, as were codes that appeared in only one dataset. ${ }^{68}$ This left 97 codes for use in the current study.

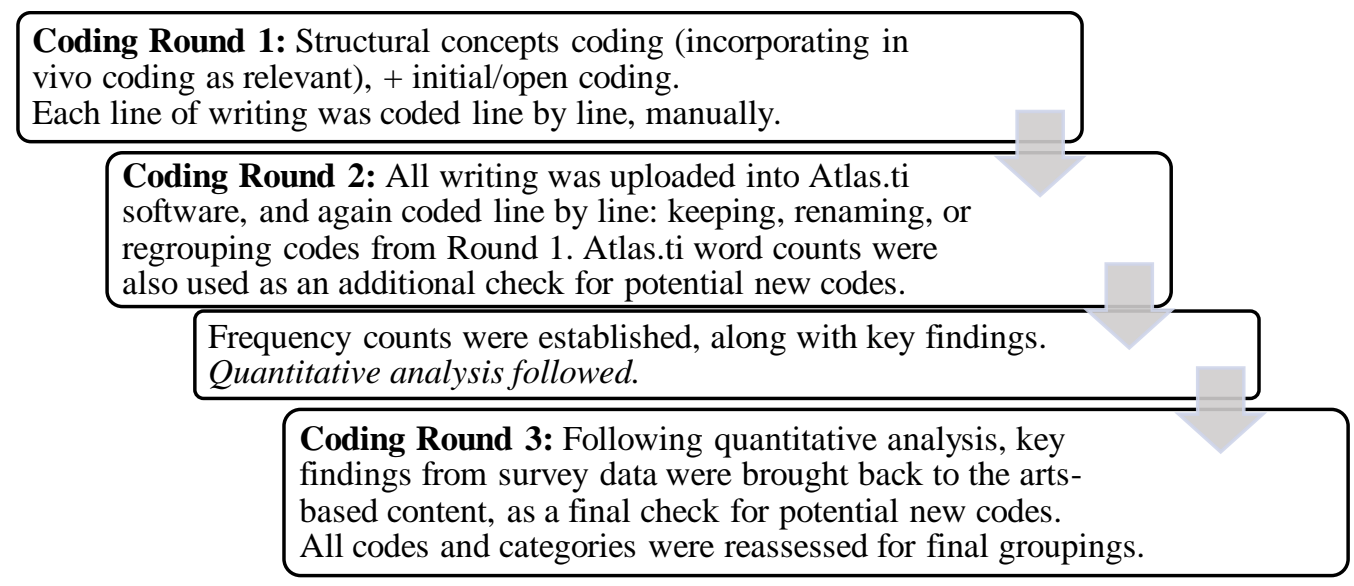

Figure 4.1: Overview of Qualitative Process of Analysis

The data set for population sample one (writings by girls incarcerated in Youth Detention Services (YDS)) utilized 89 of these codes; the second data set (entries to a writing contest associated with National Youth Violence Prevention Week) utilized 95 codes; and the third data set (a play entitled How to Start a Wildfire, written by University of Louisville students who had survived sexual violence) utilized 83 codes. These codes were grouped into eight categories:

- Interpersonal Violence

- Structural Violence

\footnotetext{
${ }^{68}$ Given that the three data sets resulted from distinct circumstances and writing prompts, it was expected that some codes would be frequent yet result from only one sample. Given the range of content, it was also expected that expressions or observations that were infrequent or rare may nevertheless provide significant insights regarding violence among young women. This additional step of analysis thus ensured that findings which may not be representative or transferrable were nevertheless rendered available (i.e., findings specific to the incarcerated population). Thus a few exceptions to the "under ten" exclusion were ultimately made, and these are reported below.
} 
- Effects (of violence)

- Barriers (to health/safety)

- Needs

- Assets

- Attributes

- Values

As analysis and discussion below will show, these themes were closely linked and interactive; they have been grouped within the four overarching categories established by RQ1, as shown in Figure 4.2:

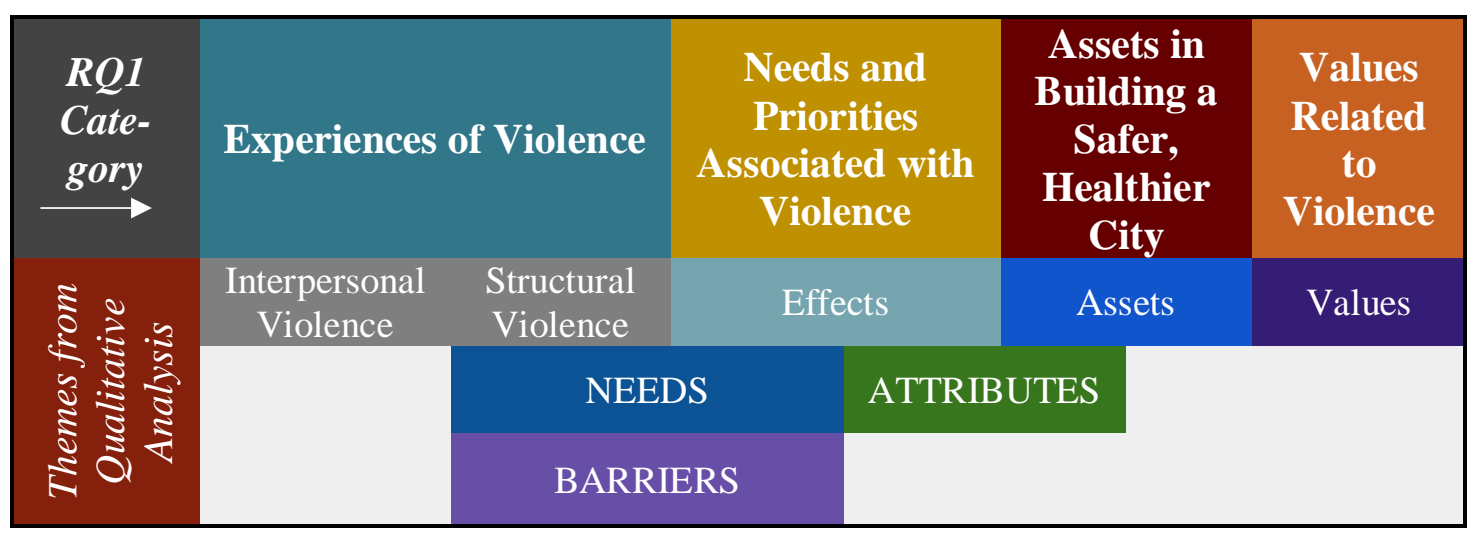

Figure 3.2: Themes grouped according to RQ1 Categories Themes falling within more than one category are capitalized.

Key findings were determined primarily via frequency counts. However, less frequent findings were noted as key when they offered unique or unexpected observations relevant to an overarching understanding of young women's experiences or perceptions of violence, safety, and well-being.

\subsection{Chapter Organization}

To organize the breadth of analyzed data, this chapter's presentation of findings utilizes headings following the list in RQ1:1) Experiences of violence; 2) Associated 
needs and priorities; 3) Values related to violence; and 4) Assets in building a safer, healthier city. For each category, the researcher prioritized findings resulting from all three populations, followed (when applicable) by findings unique to a particular dataset.

Table 4.1: Frequency Counts by Theme and Data Set

\begin{tabular}{|l|c|c|c|c|}
\hline \multicolumn{1}{|c|}{ THEMES } & $\begin{array}{c}\text { YDS } \\
\text { Program }\end{array}$ & $\begin{array}{c}\text { Contest } \\
\text { Entries }\end{array}$ & $\begin{array}{c}\text { Wildfire } \\
\text { Play }\end{array}$ & TOTALS \\
\hline Interpersonal Violence & 161 & 436 & 182 & $\mathbf{7 7 9}$ \\
\hline Assets & 149 & 188 & 132 & $\mathbf{4 6 9}$ \\
\hline Needs & 111 & 188 & 126 & $\mathbf{4 2 5}$ \\
\hline Effects & 79 & 113 & 71 & $\mathbf{2 6 3}$ \\
\hline Values & 94 & 97 & 35 & $\mathbf{2 2 6}$ \\
\hline Barriers & 59 & 70 & 25 & $\mathbf{1 5 4}$ \\
\hline Attributes & 54 & 65 & 34 & $\mathbf{1 5 3}$ \\
\hline Structural Violence & 28 & 43 & 5 & $\mathbf{7 6}$ \\
\hline Totals & $\mathbf{7 7 9}$ & $\mathbf{1 2 6 4}$ & $\mathbf{6 4 1}$ & $\mathbf{2 5 4 5}$ \\
\hline
\end{tabular}

Following the presentation of results from RQ1, this chapter addresses RQ269 by presenting key findings from the quantitative survey analyses (each population sample distinctly), and comparing them to key findings from the qualitative, arts-based data. Lastly, initial findings regarding the prototyped methods are offered, including affordances and limitations of each.

\subsection{Experiences Of Violence}

As shown in Figure 4.2, two themes that emerged from the data-Experiences of Interpersonal Violence and Experiences of Structural Violence-have been incorporated into the RQ1 category "Experiences of Violence."

\footnotetext{
${ }^{69} \mathrm{RQ} 2$ : How does this [arts-based] data, including its collection and analysis, compare to that resulting from quantitative surveys?
} 


\subsubsection{Experiences of Violence - Interpersonal}

Across the three data sets, the most reported concerns or experiences with Interpersonal Violence were domestic and intimate partner violence (D/IPV; referenced 74 times); trauma (referenced 68 times); and fear/threat (referenced 61 times). This section presents key findings regarding these three codes, followed by an overview of remaining Interpersonal Violence data.

\subsubsection{Domestic/Intimate Partner Violence (D/IPV)}

Domestic and intimate partner violence (D/IPV) was the most common experience of violence addressed in the data, which included many reports of personal experiences of D/IPV. Data also included warnings to readers based on writers' personal experiences — such as the fact that seemingly "perfect" relationships can become abusive. Many writers provided extensive detail; for example, one writer compared her relationship to a storm:

The downpour is being held up against a door by your neck because you made a mess with the dog food when you were angry.

The downpour is having your head beat into the floor for trying to stab them with the glass from the broken prom photo that you threw when you were angry.

The downpour is having your hair pulled until your scalp is bleeding because you tried to hit them back and run.

Young women additionally reported witnessing D/IPV in their homes; one writer defined violence itself as "a peek through the bedroom door crack of my mother getting 
beaten with a metal wrench, until the color of her skin turned eggplant purple." Another writer described her mother as "covered by those watercolors of pain, / smothered by those stains of bruises," while another disclosed her "fear / When The Fifth Fiancée handles my / Mother without care."

The How to Start a Wildfire script was centered specifically on sexual assault and intimate partner violence; it was thus expected that the D/IPV code would be particularly frequent for this data set. However, the script data provided only 31 percent of the total uses of the D/IPV code. Sixty-one percent were provided by writing contest data—in which D/IPV was the most frequently-occurring code not only within the theme of "Experiences of Violence," but across the entire dataset—comprising over 10 percent of all codes within contest data. The elicitation for this writing contest had been intentionally broad, prompting participants to reflect on "violence and peace, conflict and healing." The prominence of D/IPV in the resulting data is thus key to understanding how violence is experienced and perceived by young women in Louisville.

\subsubsection{2: Trauma}

Young women frequently reported a broad range of traumatizing and potentiallytraumatizing experiences; these were regularly coded in conjunction with reports of (or concerns about) child abuse, rape, sexual assault, domestic violence, or the violent death(s) of family or friends. In fact, the code trauma co-occurred regularly with other violence-related codes, particularly child abuse, grieflloss, pain, D/IPV, sexual assault/harassment, death, ${ }^{70}$ and rape. Some of these experiences were disclosed in

\footnotetext{
${ }^{70}$ Although the two codes were similar and often co-occurring, "Grief/Loss" and "Death" were kept separate to allow distinctions between references to death that did not necessarily involve personal grief/loss (i.e., "Young innocent kids and adults are getting killed over guns or drugs"), and to allow
} 
personal, graphic detail; for example, a Wildfire writer stated, "I was raped! / I was raped with my own childhood toys. / I was FOUR FUCKING YEARS OLD!" A writer incarcerated in YDS noted that her "mother gave me away / before I was even out of her womb," and a contest entrant indicated generational trauma: "I know that I will lose my baby in / The same way my mother lost her other four: / Pouring out of me before the first ultrasound, just like / Her mother and her mother and her mother before her."

Young women additionally described post-trauma symptoms, even when these were not attached to disclosures of specific traumatic experiences. For example, a Wildfire writer noted that she, like many others, "mentally cannot escape the nightmares of their assaults." A writer in YDS reported "wish[ing] I could stop / thinking about that bad situation;" similarly, a contest entrant recounted having witnessed the violent death of a friend; reporting that she "keep[s] recalling and remembering every single detail," like "the one microscopic wrinkle on [her friend's] shirt that day."

\subsubsection{3: Fear/Threat}

Young women frequently reported fear or threat that was experienced as its own form of violence, affecting women's physical or mental well-being: the "kind of fear that fills your body and turns you to stone, trapping you." While writers reported experiences with what may be labeled as social anxieties ("I'm scared of the way others feel about me"), most reports of fear or threat were associated with physical threats to life or health, such as substance abuse ("the ones I love...could [overdose] at any moment"); school shootings ("Schools here in Louisville are so scared that they are going to get shot up"); gun violence ("I can't walk on Market nor Victory Park with a certain color. I will be

distinctions between grief/loss caused by forms of separation other than death (i.e., "a legal system / Ripped me away from my father and / Disguised itself as a divorce agreement"). 
accused of belonging to a gang or 'Blast, Pop, Pop, Pop!'"); and other threats that limited young women's movements or access ("at Elliott Park...the kids can't even get in the water spout without a predator walking around looking at them in an uncomfortable way").

\subsubsection{4: Overview: Remaining Interpersonal Violence Codes}

After $D / I P V$, trauma, and fear/threat, the most frequently occurring Interpersonal Violence codes were: Loneliness/Isolation (44), Drugs/Substance Abuse (41), Guns/Gun Violence (38), Grief/Loss (37), and Bullying/Labels (37). Initially coded within the Barrier theme (as a barrier to health and safety), loneliness/isolation emerged in the data as violent in its own right. This was clear in a Wildfire writer's accusations to her mother: "you left me alone. You left me alone to be raped by your nephews... / You left me alone to have my innocence ripped away from me... You left me alone..." For YDS writers, reports of the violence of Loneliness/Isolation regularly referred to experiences within the jail environment ("When I'm alone sometimes I cry / But no one cares about why / They don't acknowledge me"), indicating an additional harm and health consequence of incarceration.

Young women frequently reported personal experiences of substance use ("I've been to rehab twice. / I detoxed in my cell") as well as experiences related to overdosing ("my mom overdosed on heroine [sic]"; "I have lost many [friends]...from mixing random pills together in a bowl"). Writers also reported having been drugged, and the fear of being drugged — which typically corresponded to disclosures of experiences or fears of sexual assault. Finally, young women referenced drugs as a general source or 
cause of violence, which they felt needed to be addressed by the city or by society:

"Young innocent kids and adults are getting killed over guns or drugs."

Reports of grief/loss in the data included writers' direct experiences of separation from loved ones, as well as their recognition and description of grief as a consequence of violence more generally. In addition, young women indicated that grief and loss can themselves be a source of harm, by causing overwhelm ("I am tired of burying dead bodies / ...I need to breath[e]") or isolation ("You wouldn't even know I lost my brother / if I didn't tell you").

Concerns about guns and gun violence were frequent and explicit, rendering a concern distinct in the data from death, murder, or grieflloss; in fact, it was also more prominent in the data than all these except grief/loss. Young women reported personal experiences with death as the result of gun violence; many also discussed gun violence in the particular context of school and mass shootings: "'[A]nother school shooting' the politician repeats / ... You [insist] again and again / that guns are not the issue at hand / how long will you accept money from the NRA?"

Young women reported bullying and labeling as sources of personal fear or threat ("I'm scared of what new things they'll say"), as well as bullying as a general issue connected to violence ("most teens....already have others bullying them"). The data also refer to the potential consequences of bullying, including suicide, self-harm, and even mass shootings ("He doesn't talk to anyone / and bullies only push him more. / No one seems to notice / a hatred building inside of him").

Frequency counts for all Interpersonal Violence codes are listed in Table 4.2: 
Table 4.2: Frequency Counts, Interpersonal Violence

\begin{tabular}{|c|c|c|c|c|c|c|c|}
\hline CODE & Count & Code & Count & Code & Count & Code & Count \\
\hline $\begin{array}{l}\text { Domestic } \\
\text { violence, } \\
\text { IPV }\end{array}$ & 74 & Grief/Loss & 37 & Rape & 22 & $\begin{array}{l}\text { Lack or } \\
\text { Loss of } \\
\text { control }\end{array}$ & 15 \\
\hline Trauma & 68 & $\begin{array}{l}\text { Sexual } \\
\text { assault; } \\
\text { Harassment }\end{array}$ & 34 & Murder & 19 & $\begin{array}{l}\text { School, } \\
\text { Mass } \\
\text { shootings }\end{array}$ & 15 \\
\hline Fear/Threat & 61 & Child abuse & 32 & Betrayal & 18 & $\begin{array}{l}\text { Emotion- } \\
\text { al abuse }\end{array}$ & 13 \\
\hline $\begin{array}{l}\text { Loneliness, } \\
\text { isolation }\end{array}$ & 44 & Death & 32 & $\begin{array}{l}\text { Coerce, } \\
\text { force, } \\
\text { trap, } \\
\text { pressure }\end{array}$ & 17 & Gangs & 10 \\
\hline $\begin{array}{l}\text { Drugs, } \\
\text { Substance } \\
\text { Abuse } \\
\end{array}$ & 41 & Pain & 31 & Silence & 17 & $\begin{array}{l}\text { Preva- } \\
\text { lence }\end{array}$ & 10 \\
\hline $\begin{array}{l}\text { Guns, gun } \\
\text { violence }\end{array}$ & 38 & $\begin{array}{l}\text { Inaction, } \\
\text { Complac- } \\
\text { ency }\end{array}$ & 28 & $\begin{array}{l}\text { Suicide, } \\
\text { Self- } \\
\text { Harm }\end{array}$ & 17 & $\begin{array}{l}\text { Participat } \\
\text {-ing in } \\
\text { violence }\end{array}$ & 7 \\
\hline $\begin{array}{l}\text { Bullying, } \\
\text { Labels }\end{array}$ & 37 & $\begin{array}{l}\text { Insecurity, } \\
\text { self-doubt }\end{array}$ & 27 & Hate & 15 & & \\
\hline
\end{tabular}

\subsubsection{5: Additional Findings and Population Disparities}

Sexual assault and sexual harassment were mentioned so frequently in contest and Wildfire data that this form of violence became key despite failing to appear at all in the YDS dataset. Child abuse, pain (references to physical or emotional pain/hurt), inaction/complacency, insecurity/self-doubt, and betrayal were all frequent and distributed across data sets. As with loneliness/isolation, the researcher initially configured inaction/complacency as a Barrier to health and safety. However, the data consistently revealed young women's perception of inaction as itself harmful —as in this description of domestic violence: "the most dangerous part / ...is having your friends in 
the hall listening to the storm play out, but making sure not to get out in it" (emphasis added).

Insecurity/self-doubt was only reported 27 times across all data sets; however, it was the most frequently occurring form of interpersonal violence reported by writers in YDS (18, or 11\%). Again, while these reports could be configured as Barriers or Needs, they include implications for health that render them significant as forms of threat experienced by young women. Insecurity/self-doubt often co-occurred in YDS writing both with loneliness/isolation and with incarceration, and typically manifested as fear and anxiety: "I am afraid of myself"; "Am I doing anything right?"; "One day just one day I want to hear that I am enough".

Death (32) was prominent as a general concern in the contest data; however, it did not appear at all in the Wildfire data set, and appeared only twice in YDS data. This disparity can be traced to the narrower theme of the Wildfire content (focused primarily on sexual assault and D/IPV), and to the expressive nature of YDS programming - in which writers were more likely to express feelings of grief/loss than to discuss death itself.

Finally, while relatively infrequent compared to the top codes, reports of lack of control (18) provided noteworthy insights regarding gendered experiences of violence. The researcher initially used in vivo coding to mark these reports as "Not my own;" this phrase and concept came up repeatedly, particularly in reference to rape and sexual assault. One writer directed her words to a perpetrator: "I guessed you had come with the right to my body;" another noted, "My body did not feel like my own." One writer described the violent nature of lacking control as follows: "I wasn't mine / i belonged to 
someone else / nothing that happened was under my control / [...] i lost my control / it was ripped out of me / I was raped."

The writing contest and the script both resulted from violence-centered prompts or concerns; by contrast, the writing program in YDS did not elicit writing specifically about experiences or perceptions of violence. Therefore, indications of violence or trauma in YDS writing resulted only when writers made individual decisions to express those concerns. ${ }^{71}$ For this reason, data from the contest and the play were consistently more detailed regarding violent experiences than data from YDS. However, Interpersonal Violence codes did ultimately comprise 21 percent of total YDS codes-compared with 35 percent of total contest codes, and 29 percent of total play codes. In other words, interpersonal violence in the YDS dataset was relatively similar in prominence to the other data sets, without being explicitly solicited. This provides some initial insight regarding the (pre-)existing prominence of violence-related concerns among girls incarcerated in YDS. Overall, codes from within the theme of Interpersonal Violence comprised 31 percent of all codes across the three data sets.

\subsubsection{Experiences of Violence - Structural}

Writers' references to Structural Violence were relatively infrequent (76) compared to their references to Interpersonal Violence (779), and references coded as Structural Violence ultimately comprised three percent of codes overall (see Table 4.3 for codes and frequency counts). Most concerns regarding structural violence were about institutional inaction — such as failures of governments, schools, or jails to respond to health- and safety-related needs. These concerns were most prominent in contest data,

\footnotetext{
${ }^{71}$ Such concerns often arose in response to prompts such as, "What would you like to change about the world, or society?" or, "What is something people don't know about you, that you'd like to share?"
} 
often co-occurring with guns/gun violence ("the weapons that no one will control") and school/mass shootings: "How do they walk out of a store with...somewhere between 33100 rounds, and then...through the playground? / Because we let them. No questions asked. No background checked." Wildfire writers additionally noted university and societal failures to hold abusers and sexual assault perpetrators accountable, at one point describing a better future as one in which institutions and the public "stop employing, promoting and electing abusers."

Table 4.3: Frequency Counts: Structural Violence

\begin{tabular}{|l|c|}
\hline \multicolumn{1}{|c|}{ Code } & Count \\
\hline Institutional inaction & 21 \\
\hline Incarceration & 17 \\
\hline Homelessness, Hunger, Poverty & 17 \\
\hline Racism, white supremacy & 10 \\
\hline Sexism, misogyny & 10 \\
\hline Rape culture & 10 \\
\hline
\end{tabular}

Incarceration was referenced primarily in YDS data, in which writers described its difficult or violent nature: "We need to take away [this jail] / Because they do not treat us right." And from another writer: "[I'm c]aught up in the system, / man they got me fucked up..."

Homelessness/hunger/poverty was also most frequently referenced in YDS data, resulting from incarcerated writers' descriptions of their own food and shelter insecurity. For example, one writer indicated that she "had to worry / about when I'm gone eat my next meal / and who house I was gone lay my head at!" Another asked, "Where am I going to live? / Where am I going to sleep? / How am I going to make money? / How am I going to eat?" The data also included references to homelessness as a general issue 
("homeless people in shelters, when there are all these abandoned houses"), and to "shelter and prosperity" as an opposite of violence.

Though less frequent, references to sexism/misogyny and racism/white supremacy offered key findings regarding ways in which young women in Louisville experience or perceive violence. Concerns regarding racism/white supremacy were often personal and detailed: "I Hate that my black can be a burden"; "I have to bleach my skin, so that I can blend in with the rest of America"; "I don't know what's worse: The fact that I'm the one making my peers uncomfortable by bringing up race, or how bringing up race makes my peers uncomfortable" (emphasis in original). Sexism/misogyny included references both to denigrations of women and to gender inequality writ large.

\subsection{Needs And Priorities Associated With Violence}

As documented in Figure 4.2 above, the RQ1 category "Needs and Priorities Associated with Violence" incorporates four of the themes derived from qualitative analysis: Barriers, Needs, Effects, and Attributes. While findings from all of these themes contribute to overarching assessments of current health and safety needs among young women in Louisville, they do so on different bases; for this reason, they are presented separately below.

It should be noted that many experiences codified below as Barriers or Needs also qualify as experiences of violence; for this reason, both were documented in Figure 4.2 as overlapping with "Experiences of Violence." Similarly, data codified as Attributes can ultimately refer to both Needs and Assets; for this reason, the Attributes theme overlaps both of these RQ1 categories. Given that barriers reported by young women overlap extensively with their reported sources and experiences of violence (just discussed), this 
theme is presented first. Young women's priorities have been determined by frequency counts, and are documented following the final theme of this section ("Attributes").

\subsubsection{Barriers}

The RQ1 category "Barriers" incorporates six codes that emerged from qualitative analysis, as documented in Table 4.4. These codes represent issues or circumstances described or implied by young women as being current or potential barriers to safety, health, or healing. Overall, codes from within the Barriers theme comprised six percent of all codes across the three data sets.

\subsubsection{Mental Health}

Mental health was the most prominent recorded barrier to health and healing in the data; it included references to anxiety, post-traumatic stress disorder (PTSD), and depression. ${ }^{72}$ Although some writers documented explicit mental illness diagnoses ("At four years old I was diagnosed with PTSD"), the data primarily include descriptions of symptoms of anxiety ("My heart's racing fast / My stomach's tied in knots / My body is perspiring") or depression ("I have bent...my chest into the shape of a coffin, something to climb into on the bad days"). Notably, some writers named mental illness without directly claiming it—an affordance of the creative medium: "suppose you were depressed 'cause / The first man / You're supposed to look up to / Tried to get between your thighs." Young women also reported experiences of mental illness in their homes, which precluded safety and well-being ("My mom would get depressed, drunk, and start fights with me").

\footnotetext{
${ }^{72}$ Note that these concerns - particularly PTSD or depression — could also be codified as effects of violence, which are documented below. This is why the "Barriers" category in Table 2 falls not only under the categories of Experiences of Violence, Associated Needs, but also under the theme of Effects.
} 
Table 4.4: Frequency Counts: Barriers

\begin{tabular}{|l|c|}
\hline \multicolumn{1}{|c|}{ Code } & Count \\
\hline Mental health & 48 \\
\hline Gender roles/expectations & 28 \\
\hline No one to depend on & 25 \\
\hline No one notices & 23 \\
\hline No one listens/hears & 19 \\
\hline Relationships: Deceptive love & 11 \\
\hline Total & $\mathbf{1 5 4}$ \\
\hline
\end{tabular}

\subsubsection{Gender Roles/Expectations}

The data indicate that gender roles and expectations had caused violence as well as fear, leading (for example) to experiences of bullying, physical abuse, and sexual assault: "They say you ugly / They say you a thot"; "I've been hurt either because I'm gay... or I look different from all the other girls." Young women also reported that gendered "training" regarding how to function in society had rendered them unable to protect themselves:

All my instincts have failed me because

My teachers taught me to do as I'm told,

My grandma not to make a scene,

My Mama to be so polite that

\section{I didn't scream.}

Another writer noted, "I was forced to do things I did not want to do, but I thought were okay. I thought it was what I was supposed to do. I felt like I was only good for what my body could offer." The data thus mark gender expectations not only as a source of violence (leading, for example, to males' perceived entitlement to female bodies), but also as a barrier among young women to their own protection, safety, and healing. 


\subsubsection{No One to Depend On}

One of the most striking findings was the frequency with which writers reported having no one to trust or depend on. This barrier to health and well-being was most prominent in YDS data, in which girls reported "learn[ing] to trust nobody" and having to take care of themselves because there was no other option. Closely linked to the need for social supports (documented in section 4.5.2.4 below), young women's sense that their safety was entirely up to them illuminates a significant barrier to both physical safety and mental health.

\subsubsection{No One Notices}

This barrier is clearly linked to "No one to depend on," but the data rendered it a distinct concern as young women reported feeling invisible or like "nobody cares." In other words, the issue for young women was not only that people around them were not dependable, but that those individuals did not even notice that there was someone or something who might need them. For example, one writer described the sense that she was "screaming but nobody hears me;" another indicated her perpetrator's inability to notice his victim in the aftermath of sexual assault: "He flops down and snores himself to sleep. I stare out the window till sunrise." The data also indicated a belief that if society were better at noticing others, it could prevent events such as mass shootings, since individuals whose "minds are darkening" could be helped before they committed atrocities. Finally, young women reported that they experienced health concerns that no one else noticed or discussed, such as miscarriages ("miscarriages aren’t an emergency because / We don't talk about them so how could we label them as such?") or gun laws that help enable school shootings ("no one will care until it happens to them"). These 
references suggest that the failure to notice can function not only as a barrier to health and safety, but as an active source of threat and ill health.

\subsubsection{No One Listens/Hears}

While similar to (and overlapping with) No One to Depend On and No One Notices, the experience of not being heard and believed—despite speaking up/out—was referenced as a distinct barrier to well-being, and as its own source of harm. With No One Listens/Hears, women's written concerns were less about going unnoticed than about being actively dismissed or muted. One writer stated, "I'll never understand why I didn't deserve to be believed. I will never understand why...my pain was not worth being heard." Other writers explicitly asked to be listened to: "I need you to be the friend who listens..."; "Ask if there is pain in my heart / Ask if there is hurt in my life... Just ask." Writers in YDS stated that "juveniles should be able to speak up on their cases [in court]"-indicating an additional need to be (and feel) heard specifically in and by the system.

Beyond their own experiences of feeling unheard, young women also reported concerns that in general, "victim's cries go unheard [ sic]"-including refugees, immigrants, victims of sexual assault and rape, and victims of gun violence.

\subsubsection{Deceptive Love}

The final frequently documented barrier to health and safety was deceptive love, which was linked throughout the data to D/IPV and sexual assault. Its frequency indicates that young women feel a sense of threat regarding all intimate relationships: "At first he seemed great we hit it off really well...Suddenly he began to change, [...] Therefore, young women, be cautious..." 


\subsubsection{Needs}

The RQ1 category "Needs" incorporates 14 codes that emerged from qualitative analysis, as documented in Table 4.5. Overall, codes from within the Needs theme comprised 17 percent of all codes across the three data sets.

Table 4.5: Frequency Counts: Needs

\begin{tabular}{|l|c|}
\hline \multicolumn{1}{|c|}{ Code } & Count \\
\hline To ACT; take responsibility & 57 \\
\hline Speaking out/Voice & 51 \\
\hline Self-worth & 49 \\
\hline Social supports & 44 \\
\hline Safety & 39 \\
\hline Self-care \& Self-love & 32 \\
\hline Access to resources & 28 \\
\hline To work together; unity & 24 \\
\hline To be heard & 24 \\
\hline To express/share & 21 \\
\hline To protect myself & 21 \\
\hline Guidance & 14 \\
\hline Education & 11 \\
\hline Empathy & 10 \\
\hline Total & $\mathbf{4 2 5}$ \\
\hline
\end{tabular}

The codes within this theme represent young women's explicit and implicit references to health and safety needs they experienced or recognized in their own lives, in their community, or in society. As the experts of their own lived experiences (Curtis, Bryce, \& Treloar, 1999), young women's assessments and articulations of their needs form the cornerstone of adequate and appropriate responsive action. This section presents findings regarding the top five reported needs, followed by an overview of the remaining nine. 


\subsubsection{To ACT; Take Responsibility}

Young women's demands, pleas, and encouragements to take action and responsibility regarding violence and suffering were most prominent among expressed needs. The capitalization of the word "ACT" in this theme is reflective of the frequency with which writers communicated urgency via capitalization, exclamation marks, rhetorical weight, and repetition. Young women reported both fatigue and outrage at leaders' failures to act and indicated specific action steps leaders should take. These included: educating all genders about consent and sexual assault, creating a curfew, changing how youth in the justice system are treated, requiring full background checks for gun purchases, holding abusers accountable, "address[ing] the cultural epidemic of toxic masculinity," cleaning up litter in under-resourced neighborhoods, and following through on their promises regarding safety and health.

Writers in the study also recorded perceived needs by describing actions they were already taking, which implied their value and applicability. These actions included feeding the homeless, calling out racism, "planting seeds of love," and advocating for other victims.

Most prominent in the data were writers' encouragements directly to their readers_ - including other young women ${ }^{73}$ - to take action in response to violence. Some of these were general, such as urging readers not to "sit back and wait for change. / Just MAKE THE CHANGE!" or urging readers to "fix the violence," "make a change in the world," or "fight for safety." Others were more specific: "here's what I need you to do... / I need you to knock on that door when you hear that couple fighting;" "Let [your sisters]

\footnotetext{
${ }^{73}$ Much of this writing was inclusive of the author as well; young women seemed rhetorically to include themselves among those they were urging toward (continued) action.
} 
know that they can always say no." Young women also asked that their readers: write to senators, examine their own toxic behaviors, spread the word about the need to stop violence, "help get rid of gangs," listen to others, learn coping skills, join protests, "be a good role model," actively support other women, "help your friends choose right," and avoid being mean to those they do not like.

\subsubsection{Speaking Out/Voice}

Echoing the barrier of not being heard/believed, writers expressed speaking out and having a voice as essential needs for improved health and safety. The data include writers' descriptions of having spoken out in moments of sexual violence: "PLEASE DON'T TOUCH ME"; "Look, I'm a virgin and I'm not really comfortable with that idea." Writers also indicated that speaking was a fundamental means of preventing or ending violence: "Your voice as powerful as your fist;" "I feel that my voice can change others;" "Show your sisters how to say what they think;" "if your boyfriend or husband abuses you speak up make a statement it doesn't matter how."

Notable in the data were frequent uses of "speaking" or "having a voice" as a synecdoche standing in for valued personhood. Having and using one's voice emerged as an essential aspect of dignity, and this dignity was implied as a first step toward change: "I believe that all people have a voice;" "I do have a... / voice that I feel needs to be heard;" "Now I'm finding my way, / My light / My voice;" "My voice will in fact be heard." The prominence of voice among young women's assessments of violence-related needs aligns with the most prominent forms of violence in the data (D/IPV, trauma, loneliness/isolation, fear/threat), as well as the prominent barriers of not being believed, not being noticed, and not being supported. 


\subsubsection{Self-Worth}

Young women's indications of the need for self-worth were often indirect or implicit—emerging as writers described their own healing process ("I learned that I am valuable;" "I realize I mean more than what people think"), or as they encouraged others: "Just be who you are;" "I am scarred. But I am more—and so are you." Self-worth also emerged as a clear need when young women explicitly noted its lack: "My standards were low / and my self-worth even lower;" "So young I started to get off track, / No selfworth;" "I felt like I was only good for what my body could offer;" "I kinda hate myself;" "I can't wait to be surrounded by thoughts of acceptance." Finally, self-worth became apparent as a need through the frequency with which pride came up in content about young women's health and safety: "I now realize that I took all that crap, / And I'm way better than that;" "You may talk down about me, / but I'll never let it stop my process."

\subsubsection{Social Supports}

The data are clear that young women experience and perceive social supports as significant needs - essential to their sense of joy, acceptance, meaning, and belonging, all of which are aspects of well-being. Similarly, social supports were represented as means of being seen and heard which, as noted above, had been indicated as prominent aspects of health. (One cannot be or feel heard if there is no one there to listen.) Young women also described social supports as guides and as protective forces: preventing violence, intervening in violent or difficult situations, and helping the healing process. Notably, the need for social supports was most prominent by far in the YDS data; this aligns with the prominence in that dataset of loneliness/isolation, insecurity, and no one to depend on. 


\subsubsection{Safety}

Key to this study was the frequency with which young women documented their lack of safety, and the varied ways in which this lack manifested in their lived experiences. Writers reported hiding who they really were, and "becom[ing] heartless" in order to avoid betrayal or bullying. They also reported having lacked safety in hospitals - in which they had to "prove [their] pain" to doctors after a rape — as well as in schools, due to threats of mass shootings, bullying, fights, and sexual assault. Writers additionally noted that they lacked safety when asserting themselves in dangerous situations and when reporting their own victimization.

Finally, young women's descriptions of what they wanted implied what they currently lack (and thus need): "I want to feel like I'm protected by my home;" "we [teens] should have a safe place to go;" "A park is supposed to be a place where kids feel safe to go and play and don't have to worry;" "I want a good president. / I want good laws and actions;" "I want a home that I feel safe at;" "I want to live in the city that never sleeps / But not just because we're kept awake by gunshots."

\subsubsection{Overview: Remaining Needs}

Remaining needs can be seen in Table 6. Self-care/self-love was kept distinct from self-worth due to the frequency with which the specific actions of "loving myself" or "car[ing] about herself" occurred in the data. Access to resources often aligned with safety, in that young women's access to various resources (education, parks, health care) was limited by their lack of safety. However, the data also indicate specific resources young women deemed significant for health, safety, or healing, including: counseling, employment, shelter, "community spots" for teenagers, support groups, health care, and 
general opportunities. The need to be heard aligns with the barriers no one notices and not being heard/believed; it also overlaps to an extent with speaking out/voice. However, the data indicate that many young women perceived speaking out as its own necessary, powerful action that can be distinct from actually having been heard. Similarly, the need to express/share was kept distinct from speaking out. This is because, according to the data, young women's need to express what happened to them was about telling their story as a means of better understanding or processing it (for themselves). By contrast, speaking out was more about accessing help from—or stimulating change in —others.

Finally, it should be noted that young women's indications of the need for education referred specifically to education regarding bullying, consent, sexual assault, cultural differences, and the consequences of violence.

\subsubsection{Effects}

The RQ1 category "Effects" incorporates 14 codes that emerged from qualitative analysis, as documented in Table 4.6. Overall, codes from within the Effects theme comprised 10 percent of all codes across the three data sets.

Table 4.6: Frequency Counts: Effects

\begin{tabular}{|l|c|}
\hline \multicolumn{1}{|c|}{ Code } & Count \\
\hline Guilt/Shame/Regret/Self-Blame & 42 \\
\hline Hopelessness, Overwhelm & 34 \\
\hline Lingering effects & 28 \\
\hline Bruises, Scars & 24 \\
\hline Anger/rage & 23 \\
\hline Pretending, hiding & 21 \\
\hline Tears & 16 \\
\hline Memories & 15 \\
\hline Sensory details & 15 \\
\hline Uncertainty & 15 \\
\hline Numb/numbing/numbness & 12 \\
\hline
\end{tabular}




\begin{tabular}{|l|c|}
\hline Scream/can't scream & 10 \\
\hline "I had to grow up too fast" & 5 \\
\hline The BODY speaks & 3 \\
\hline Total & $\mathbf{2 6 3}$ \\
\hline
\end{tabular}

These codes represent young women's references to experiences that followed violence, either as physical reactions (bruises, tears) or as longer-term outcomes (hopelessness, memories). Recognizing the effects of violence as described by young women is essential both for fuller assessments of harm caused by violence and its barriers, and for determining adequate responses. This section presents findings related to the top five reported effects, followed by an overview of the remaining 10 .

\subsubsection{Guilt/Shame/Regret/Self-Blame}

Some form of guilt/shame/regret/self-blame was the most commonly reported effect of violence that also had a relatively even distribution across the three data sets. Young women described current regret ("Done got pregnant at 16 / Soo much stuff I dreamed / of doing / now it's ruined"), as well as beliefs that shame or guilt is unfounded: "I want you to know that [the sexual assault] wasn't your fault, and you don't have to feel like it was." The data also indicated shame as an immediate mental response to victimization: "He gets up to clean off. I turn on my side. Shocked. Lost. Ashamed..."

\subsubsection{Hopelessness/Overwhelm}

References to hopelessness/overwhelm were equal in number to those of guilt/shame/regret, but were less evenly distributed - appearing only in YDS and contest data. Young women frequently reported beliefs that violence "is everywhere and it's gotten out if control [sic]"—contributing to a sense of overwhelm, fatigue, numbness, and hopelessness: "That's just how it is on this bitch of an earth." Other writers referred 
to hopelessness less as a result of the prevalence of violence in general than of their own experiences: "I am sick of life /...I'm ready to give up / I've seen and done too much / just to be 17 like fr."74

\subsubsection{Lingering Effects}

Young women reported long-term effects of violence (lingering effects), which included "emotional scars," the inability to generate satisfying relationships, traumatizing memories ("The memory of a phone camera flash from behind me as my body was being penetrated and violated still hurts and haunts me today"), lack of memory, and mental health concerns ("Some girls get raped when they are young, so that carries with them when they are older, so that makes some girls depressed and wanna commit suicide").

\subsubsection{Bruises/Scars}

Young women regularly incorporated the presence of bruises or scars into references to personal or witnessed experiences of violence: "She [my mother] was a canvas... / of purple and brown and black, smothered with messy stains of bruises." Notably echoing the barriers no one notices and not being believed, the visibility of bruises or scars was a significant concern in the data - as young women were again worried about being believed: "We live in a world where we're told...'bruises/physical trauma/evidence or it didn’t happen'"; "there are bruises where no one can see them"; "I've been abused / but I don't have bruises." The data make clear that the forms of violence that cause bruises or scars are not necessarily a greater concern for young women than the violence(s) of dismissal, disbelief, and apathy.

\footnotetext{
${ }^{74} \mathrm{Fr}$ is used by some writers to mean "for real."
} 


\subsubsection{Anger/Rage}

Young women variously referenced anger or rage as: visceral effects of violence ("This is vomit and semen...This is saying no...This is rage and hate and fear"); as righteous responses to violence ("people are very angered... / We must fight"); and as necessary aspects of justice and healing: "Start a fire so huge and hot and full of rage that it cannot be ignored." Young women also expressed a fear of anger or rage, perceiving it as an emotion that leads to lack of control and abuse. Considering that several writers encouraged women to embrace rage, it is clearly an important aspect of preventive and responsive health and safety strategies; researchers should therefore work to generate further insight around this complex emotion and the gendered nature of its expression and perception.

\subsubsection{6: Overview: Remaining Effects}

Writers in this study commonly reported hiding their experiences of victimization or their responses to it (pretending/hiding). This response is often linked in the data to shame and to lack of trust in those to whom disclosures might be made (connected with not being believed); it can therefore also be linked to the need to protect myself. Other reported effects were physical and mental responses often associated with violence and trauma: tears, memories, numb/numbing/numbness, scream/can't scream. Young women also referenced uncertainty ("I wonder what will happen next")—a particularly prominent concern for YDS writers.

"I had to grow up too fast" was an in vivo code marking an infrequent but nevertheless repeated and memorable finding: young women believe that violence requires them to grow up quickly, losing the opportunity to be a child: "at twelve I had to 
be / Fifteen, and at seventeen / I have to be twenty." Finally, young women noted that even when they could not remember specifics regarding their trauma, their bodies remembered-leading to frequent sickness and other symptoms of PTSD (the BODY speaks).

\subsubsection{Attributes}

The "Attributes" theme includes codes that may be applied to RQ1 categories "Needs" or "Assets. It incorporates six codes that emerged from qualitative analysis, as documented in Table 4.7. Overall, codes from within the Attributes theme comprised six percent of all codes across the three data sets.

Table 4.7: Frequency Counts: Attributes

\begin{tabular}{|l|c|}
\hline \multicolumn{1}{|c|}{ Code } & Count \\
\hline Innocence & 42 \\
\hline Authenticity / Inner self & 40 \\
\hline Claiming or Defining & 30 \\
\hline Vulnerability, weakness & 15 \\
\hline Beauty, beautiful & 13 \\
\hline Smart, not smart & 13 \\
\hline \multicolumn{1}{|c|}{ Total } & $\mathbf{1 5 3}$ \\
\hline
\end{tabular}

These codes represent young women's references to personal attributes and selfperceptions, which inevitably informed the writers' perceptions of health, safety, violence, and conflict. An understanding of how young women perceive themselves will contribute to better approaches to improving their health and safety. This section presents findings related to the two most-documented attributes, followed by a brief overview of the remaining four. 


\subsubsection{Innocence}

Innocence appeared primarily in contest data (15), in which the concept of innocence - particularly that of children and babies — was used to underline the tragedy of violence: "Young innocent kids... are getting killed;" "How are we to live knowing the innocent and righteous die...?;" "it is our babies that are being hunted." The concepts of innocence and childhood were also employed to emphasize the ubiquity of violence: "From the time we're just kids, we get teased on the bus and kicked around in the hallways." In YDS writing, innocence and childhood were means of speaking to the injustice of incarceration and subsequent labels: "Don't call me a criminal, call me a child / who made mistakes." Finally, innocence was often referenced in Wildfire to underline the abhorrent nature of child sexual abuse: "We have to do better. For every little one who doesn't know what assault means so that they never ever have to."

\subsubsection{Authenticity/Inner Self}

Young women regularly identified the process of finding and embracing their authentic selves as a necessary aspect of health and healing: "I am figuring out who I truly am. / I am learning how to love myself;" "I need to find myself." Similarly, losing one's true self was documented as an aspect or effect of violence: "Two years...given away to drugs / Changing who I was;" "I've lived 17 years...feeling ashamed of who I am and what happened to me" (emphasis added). The apparent necessity in the data of feeling connected to oneself illuminates this connection as a significant health asset (when present), and a health need (when lacking).

Notably, 75 percent of references to an authentic or inner self appeared in the YDS dataset (28), in which writers regularly depicted their true selves as hidden or 
unseen: "I wish people knew the real Me;" "I'm a good person inside;" "I want people to see a part of me that I'm scared to show." This aligns with the frequency in the YDS data of loneliness/isolation, no one listens/hears, and no one to depend on.

\subsubsection{Identity Claiming/Defining}

Young women across the data regularly used the writing opportunity to claim identities or define who they were: "I am a strong black goddess;" "I am NOT a victim of violence. I am a SURVIVOR!;" "I mean more than what people think. / I AM ME!" Given the significance in the data of feeling connected to (and recognized for) one's authentic self, the opportunity to make clear statements about one's identity appears be important to young women's sense of health, safety, and well-being.

\subsubsection{Overview: Remaining Attributes}

Young women documented vulnerability/weakness - what they perceived to have rendered them susceptible to violence-including: being small in size, showing their feelings, being kind to others, having been taught deference, or not having been taught "how to rage." The concept of beauty was also frequently cited in the data-particularly by writers telling other women that they are beautiful. Though often used in unconventional ways (such as to refer to beautiful souls, or to celebrate nontraditional beauty), the frequency of the term or concept of beauty/beautiful highlights the felt importance of this potential descriptor among young women.

\subsubsection{Priorities}

Using frequency counts and findings from RQ1 Categories One and Two, it is clear that domestic/intimate partner violence $(D / I P V)$ and trauma were priorities for young women when it comes to violence, safety, and health. Beyond these experiences of 
violence, and regardless of the particular forms of violence discussed, young women prioritized being heard, acknowledged, and believed. This links directly to D/IPV and trauma, as both are issues or experiences about which women have historically encountered doubt or dismissal (Randall, 2010; Jordan, 2004). Young women's need to be heard and believed — and their sense that this need has not been met—is also connected to all of the other prominent issues in the data, including fear/threat, loneliness/isolation, guns/gun violence, the violence of inaction, young women's felt need to speak out or express, their lack of social supports, their need for access to resources, and structural issues such as sexism and institutional inaction.

\subsection{Assets In Building A Safer/Healthier City}

As documented in Figure 4.2 above, the RQ1 category "Assets in Building a Safer/Healthier City" incorporates two of the themes derived from qualitative analysis: Assets and Attributes. Because the Attributes theme was discussed above (under "Effects"), this section strictly covers Assets.

\subsubsection{Assets}

The theme of "Assets" incorporates 13 codes that emerged from qualitative analysis, as documented in Table 4.8. Assets were identified as elements, attributes, or states that were depicted as supporting or signifying health or healing. Overall, codes from within the Assets theme comprised 18 percent of all codes across the three data sets.

Table 4.8: Frequency Counts: Assets

\begin{tabular}{|l|c|}
\hline \multicolumn{1}{|c|}{ Code } & Count \\
\hline Confidence, Empowerment & 70 \\
\hline Hope & 69 \\
\hline
\end{tabular}




\begin{tabular}{|l|c|} 
Encouraging/Advising/ & \\
Advocating for others & 55 \\
\hline Resilience & 52 \\
\hline Perseverance, Persistence & 48 \\
\hline $\begin{array}{l}\text { Imagination/Dreams (for the } \\
\text { future) }\end{array}$ & 32 \\
\hline Family & 30 \\
\hline Support from others & 23 \\
\hline Healing, Transformation & 23 \\
\hline Strength & 22 \\
\hline Courage, Leadership & 19 \\
\hline Learning from trauma/pain & 15 \\
\hline Forgiveness & 11 \\
\hline Total & $\mathbf{4 6 9}$ \\
\hline
\end{tabular}

It should be noted that although this RQ1 category mentions a "safer/healthier city," the researcher also documented assets that supported individual and interpersonal health and healing - recognizing that improved health at these levels can itself become an asset in improving overall community health. Finally, the 13 assets typically emerged explicitly as assets; however, writers' perception of these elements was at times implied; i.e., when lack of a given element was depicted as an impediment to health or healing. This section provides details regarding the top five assets, followed by an overview of the remaining eight.

\subsubsection{Confidence, Empowerment}

Young women across the data depicted themselves, other women, and other youth as necessarily or inherently confident and empowered. Most prominent were writers' descriptions of themselves as confident or powerful: "Call me confident;" "I have more balls than a man ever has;" "I have the control to determine who I want to touch me;" "We are proud of Our round hips;" "You have no power over me;" "I love my melanin and my melanin will never change for anyone;" "you will / See how / I rise like dust / 
Like Maya;" "I won't sit back, shut up, and let you stay comfortable." Reflecting identity claiming/defining, writers also communicated confidence by claiming empowered identities: "I'm the protagonist;" "I am my own person;" "I’m a woman, / born a powerful queen."

Young women also noted confidence and empowerment as necessary aspects of both healing and safety: "loving myself...helped to give me the confidence to own my life;" "I remember the day I finally said NO. / I found my beauty and loved everything about myself, / Head-to-toe." This confidence occasionally included self-protection that edged toward a form of revenge: "Live in awe / And in fear of me;" "I will haunt you."

Finally, writers underlined the importance of confidencelempowerment through the frequency with which they encouraged it in their readers: "your story is powerful, and it can transform;" "When they say 'you can't,' what they mean is, 'you can...';" "b the woman who always proud to be who they are [sic];" "Respect is something you Demand / by the way you walk and the way you Talk." The prominence of confidencelempowerment in the data, in the context of violence-related content, indicates its importance for improved health and safety among young women.

\subsubsection{Hope}

Young women reported hope by explicitly claiming the word for themselves or their actions ("I hope for the better;" "I am not despair...Nahhhhhhh, you see I'm hope"); they also reported hope through indications of belief in the possibility of change: "I'll rise above all this;" "I understand the world's evil / I say that people will change;" "Now is your chance to make a change in the / World." Young women also documented hope via visions for a positive future: "My vision for the future is not only for Louisville but also 
for the world;" "The rain stings, yet heals, / The grass grows... / There is hope;" "The fire you build will ignite the things that have dried up and died in your mind." Finally, writers reported hope by expressing confidence in their own ability to contribute to positive change: "I believe that I can make a difference;" "I refuse to let it be;" "They'd all say my life was over / but, little did they know / it just began."

Not only did hope emerge as one of the most frequently occurring codes in the data (across all categories), but it is also fundamentally linked to all of the other top assets: confidencelempowerment, encouraging/advising others, resilience, perseverance/persistence, and imagination/dreams for the future (Huen et al., 2015; Snyder, 2002). Consequently, hope is clearly key to a fuller understanding of young women's experiences and perceptions of violence.

\subsubsection{Encouraging/Advising/Advocating for Others}

Much of the writing across the data was outward-facing: designed to correct readers' assumptions, stimulate action, or to help readers who may need encouragement and support. Young women's encouragements ranged from affirming statements ("You're beautiful you're smart; "You have so much potential;" "You have the ability to heal") to specific advice: "Dudes is not worth losing happiness;" "if your partner abuses you find help;" "I want you to know that it wasn't your fault, and you don't have to feel like it was." Writers also included general statements of support for other women: "You were born to be smart and wise women;" "women can do anything that a men [sic] can do;" "Women / We have to stand up for ourselves." Finally, the data include writers' indications of their current efforts to encourage and advise: "I spread self-love 
everywhere;" "I’ve been able to help guide sisters...to seek access for the things they need;" "I can give good advice."

\subsubsection{Resilience}

During analysis, the term resilience was used to code indications or descriptions of having come through—or having "bounced back" from—something difficult: "Being abused affected me heavily but I'm bouncing back and keeping my head up." Closely linked both with hope and with perseverance/persistence ("my hope—my story—are a part of the thing that makes me resilient"), resilience as a concept indicates not only having persevered, but having come through that perseverance (to some extent) in order to realize a level of equilibrium or healing. Young women often used metaphors when depicting resilience: "We have learned to make beauty out of blood;" "gotta keep my head above water / even though it's fucking cold;" "I knew I could open my wings \& fly again"_-indicating that metaphor may be an important means by which to understand

what healing means to young women. Writers also documented the importance of using difficult histories to create something good: "Be the woman that will fail and will rise up even stronger;" "The fire you build will ignite the things that have dried up and died in your mind;" "I'll rise above all this / I'm much stronger than before."

\subsubsection{Perseverance/Persistence}

Although closely linked to resilience, the frequency with which writers documented perseverance and persistence rendered it a separate finding. Young women indicated the labor and difficulty that goes into well-being ("I've tried for a long time, a very long time indeed;" "I will push past / Those of you who oppose me"), and encouraged readers to keep going despite the difficulty: "Don't you ever listen to people 
who want you to give up on your dream." Finally, young women indicated the importance of commitment (to others and to causes) as a form of perseverance: "I create a better world for you to live in. / This is my promise;" "We will not be deterred;" "I will be the flashing light / You see at night and / The flashing light you see / During the day / I will not go away."

\subsubsection{Overview: Remaining Assets}

Beyond the top five assets, young women indicated the ability to imagine and dream futures, which is critical to establishing, maintaining, or communicating hope: "This [city] could be a place of opportunities in heaps / Somewhere for us to feel safe visiting our favorite spots...". Echoing the need for social supports (addressed above), writers also reported that their existing support from others had been an asset to health, safety, and healing ("once I seen your face / I knew to keep going"). Family was also noted as a positive source of connection or support, particularly among writers in YDS, whose writing provided 70 percent of family codes. These young women often expressed desire to be back with their families, or to be able to better help their families: "Every time I'm in jail, I feel... / helpless... / I wish I could help my momma / and my brothers / and my sisters / I would be there for them..."

Young women additionally described or referred to their own healing; the data indicate that having experienced some level of healing is an asset in that it supports further health and attainment. In addition, sharing the experience of healing emerged as a means by which individuals help others; this indicates the potential significance of peer education and support. 
Descriptions of strength, desires to be strong, or claims of strength were frequent in the young women's writing, supporting the prominence of both

perseverance/persistence and confidencelempowerment. Writers also frequently noted the need for courage and leadership — and aligned with resilience in the importance they attributed to learning from trauma. While less frequent in the data, forgiveness also emerged as a health asset, particularly for its ability to support one's sense of self and agency: "I forgave him because I have control—not him;" "I want to forgive [my past self, who was assaulted]. I want to love her. I broke my silence because I believe her."

\subsection{Values Related To Violence}

As documented in Figure 2 above, the RQ1 category "Values Related to Violence" incorporates the aligned theme of Values, which emerged during analysis. The Values theme includes 12 codes, as documented in Table 4.9; this section offers a brief overview. Overall, codes from within the Values theme comprised nine percent of all codes across the three data sets.

Table 4.9: Frequency Counts: Values

\begin{tabular}{|l|c|}
\hline \multicolumn{1}{|c|}{ Code } & Count \\
\hline Caring for others & 37 \\
\hline Justice, injustice & 28 \\
\hline Trust, Loyalty & 26 \\
\hline Faith / God & 19 \\
\hline Kindness & 17 \\
\hline Respect & 16 \\
\hline Beliefs & 15 \\
\hline Light vs Darkness & 15 \\
\hline Love & 15 \\
\hline Standing up & 15 \\
\hline "shouldn't have to" & 13 \\
\hline Diversity & 10 \\
\hline Total & $\mathbf{2 2 6}$ \\
\hline
\end{tabular}


Young women indicated that caring for others was a significant value for them; this was most apparent in explicit statements about this value and how it informs their actions: "I want the people around me to feel positive energy;" "If I could take your pain and bear it for you, I would;" "I make my own money (job) to buy my own things, to help my mom out...". Caring for others emerged most frequently in the YDS dataset (28), in which writers often expressed concern for others as an aspect of presenting their authentic self. ${ }^{75}$

Justice/injustice emerged in the data as young women discussed the justice system itself ("police need more evidence before charging;" "I went through a process to seek justice"); however, it was most often discussed in the context of fairness: "What she fears is...working just as hard as any man, if not more, only to get paid less;" "we, the youth, are the ones...paying for their [politicians'] villainous wrongdoings"). Young women's concerns that abusers are not held accountable for their actions, which has been mentioned above, also fell within the value of justice/injustice: "When does this stop? / When sexual assaulter Brock Turner can be released from jail after 3 months...?"

Young women identified trust and loyalty as significant values, often by highlighting the violence of betrayal (discussed in Category 1) and implying the need and desire for its opposite. However, writers were also explicit about their positive drive toward trust and loyalty: "Healing is about honesty and trust;" " I wish I could help my momma / and my brothers / and my sisters / I would be there for them / I would ride for them." Similarly, faith as a value primarily emerged as young women described their sense of separation from it: "is there even a GOD or a plan for me?;" "With so much evil

\footnotetext{
${ }^{75}$ Authenticity/inner self (in the Assets theme) was most prominent in the YDS data set.
} 
in this world / it's hard to have faith;" "So, I'm sorry, but I can't believe in God;" "I wanted to be something in life / guess it's not meant for me / guess this the plan god got for me."

Although the remaining values in Table 4.9 were relatively straightforward, "shouldn't have to" emerged during in vivo coding as a version of justice/injustice. Young women's uses of this phrase (and similar) were relatively infrequent, yet consistently powerful and concise: "I had to worry / about when I'm gone eat my next meal / and who house I was gone lay my head at! / Like no kid should have to be worried about all that;" "I shouldn't have to hear [terrifying words] everyday;" "They worry about shooters at school. Or what's going to happen to their family. People shouldn't have to worry about this."

The 12 values that emerged in analysis illuminate potential assets; they may also help develop better understandings of how young women are affected by various forms of violence. ${ }^{76}$

\subsection{Quantitative Findings}

Having delineated what young women's art reveals about their experiences and perceptions of violence (RQ1), the following section is designed to begin answering RQ2: How do these data, including their collection and analysis, compare to those resulting from quantitative surveys? Because each of the three population samples corresponded with distinct quantitative data sets, results from quantitative analyses are organized in this section according to sample. As findings are presented, they are correlated or juxtaposed with the results of qualitative analysis offered in sections two

\footnotetext{
${ }^{76}$ For example, if caring for others and trust/loyalty are significant values, how might this affect the level of trauma caused by sexual assault by a known perpetrator?
} 
through five above. Section 4.9 will then provide initial findings regarding the methods utilized, including a comparative assessment of the three prototyped methods.

\subsubsection{Females Incarcerated in YDS}

As noted in Chapter Three, the YDS sample is the only one for which fullycorresponding survey data were obtained — allowing direct comparison to the writers' creative output. Twenty-four of the 27 program participants consented to this study and completed questionnaires, from which the following results were drawn.

\subsubsection{Demographics}

Demographic information for study participants is documented in Table 4.10.

Table 4.10: Demographics: YDS

\begin{tabular}{|c|c|c|c|}
\hline \multicolumn{2}{|c|}{$\begin{array}{l}\text { Demographic } \\
\text { Variable }\end{array}$} & \multirow{2}{*}{$\begin{array}{l}\mathbf{N} \\
4\end{array}$} & \multirow{2}{*}{$\begin{array}{c}\begin{array}{c}\text { Percenta } \\
\text { ge }\end{array} \\
16.7\end{array}$} \\
\hline Age & 14 & & \\
\hline & 15 & 6 & 25.0 \\
\hline & 16 & 7 & 29.2 \\
\hline & 17 & 7 & 29.2 \\
\hline & Total & 24 & 100.0 \\
\hline \multirow[t]{8}{*}{ Race } & Black & 12 & 50.0 \\
\hline & White & 5 & 20.8 \\
\hline & Hispanic/Latina & 2 & 8.3 \\
\hline & Mixed: Asian, White & 2 & 8.3 \\
\hline & Mixed: Black, Hispanic & 1 & 4.2 \\
\hline & $\begin{array}{l}\text { Mixed: Black, White, } \\
\text { Hispanic }\end{array}$ & 1 & 4.2 \\
\hline & Mixed: Black, White & 1 & 4.2 \\
\hline & Total & 24 & 100.0 \\
\hline \multicolumn{4}{|c|}{$\begin{array}{l}\text { Sexual } \\
\text { Orient- } \\
\text { ation }\end{array}$} \\
\hline & Straight & 15 & 62.5 \\
\hline & Lesbian & 5 & 20.8 \\
\hline & Bisexual & 3 & 12.5 \\
\hline & Missing & 1 & 4.2 \\
\hline & Total & 24 & 100.0 \\
\hline
\end{tabular}




\subsubsection{Results: ACEs}

The YDS survey begins by screening for adverse childhood experiences (ACEs); such screenings result in a score ranging from zero to 10 . Over half $(56.5 \%)$ of YDS study participants reported four or more ACEs, compared to 15.2 percent of participants in the original ACEs study of Kaiser Permanente customers ( $\mathrm{n}=17,337$; Anda \& Felitti, 2012). ACEs research indicates that a score of four or higher is associated with an elevated risk for adverse health outcomes across the life course (Aces Too High, 2017). In addition, 47.8 percent of YDS participants reported five or more ACEs, which indicates an elevated risk for complex trauma (Sherman \& Balck, 2015). ${ }^{77}$ Finally, 34.8 percent of YDS participants reported six or more ACEs; in previous studies, individuals with six or more ACEs were shown to have "had 24.36 times increased odds of attempting suicide" (SAMHSA, 2018).

\footnotetext{
${ }^{77}$ Complex trauma is defined by The National Child Traumatic Stress Network as “both children's exposure to multiple - often of an invasive, interpersonal nature - and the wide-ranging, long-term effects of this exposure" (NCTSN, 2018).
} 
Table 4.11: Specific ACEs and their Frequencies

\begin{tabular}{|c|c|c|}
\hline $\begin{array}{l}\text { ACEs } \\
\text { Number }\end{array}$ & ACEs Questions (Taken directly from the questionnaire) & $\mathbf{N}$ \\
\hline 1 & $\begin{array}{l}\text { Have you often experienced being insulted or } \\
\text { sworn at, or being put down verbally? }\end{array}$ & 16 \\
\hline 2 & $\begin{array}{l}\text { Have you ever been the victim of violence (physical } \\
\text { abuse, mugged, or assaulted)? }\end{array}$ & 12 \\
\hline 3 & $\begin{array}{l}\text { Has anyone touched you inappropriately, or } \\
\text { sexually abused you? }\end{array}$ & 8 \\
\hline 4 & $\begin{array}{l}\text { Have you often felt that no one in your family loved } \\
\text { you, or looked out for you, or felt close to you? }\end{array}$ & 13 \\
\hline 5a & $\begin{array}{l}\text { Have you ever experienced not having enough to } \\
\text { eat, had to wear dirty clothes, or had no one to } \\
\text { protect you? }\end{array}$ & $\begin{array}{c}11 \\
(5.5)\end{array}$ \\
\hline $5 \mathbf{b}$ & $\begin{array}{l}\text { Were your parents/guardians ever too drunk or high } \\
\text { to take care of you? }\end{array}$ & $6(3)$ \\
\hline 6 & $\begin{array}{l}\text { Were your parents/guardians ever separated or } \\
\text { divorced? }\end{array}$ & 18 \\
\hline 7 & $\begin{array}{l}\text { Has your parent ever been pushed, grabbed, } \\
\text { slapped, or had something thrown at them, or hit so } \\
\text { hard it left a mark? }\end{array}$ & 13 \\
\hline 8 & $\begin{array}{l}\text { Have you ever lived with someone who had a } \\
\text { problem with alcohol or drug addiction? }\end{array}$ & 10 \\
\hline 9 & $\begin{array}{l}\text { Have you ever had a household member who was } \\
\text { mentally ill or depressed, or attempted suicide? }\end{array}$ & 10 \\
\hline \multirow[t]{2}{*}{10} & $\begin{array}{l}\text { Have you ever had a household member go to } \\
\text { jail/prison or be deported? }\end{array}$ & 17 \\
\hline & Total & 125.5 \\
\hline
\end{tabular}

Participants' answers to particular ACEs questions indicate their experiences of violence; these are therefore noted in Table 4.11, along with the frequency with which they occurred. The most common adverse childhood experiences were parental divorce/separation $(n=18)$, incarceration or deportation of a family member $(n=17)$, and being verbally abused $(n=16)$. Over half of participants had also witnessed domestic violence in their home $(n=13)$ or felt that no one in their family cared about them $(n=$ 13). These findings echo girls' writings; though writers only infrequently indicated absent parents, they frequently implied verbal abuse and lack of concern from others (as seen in the code no one to depend on). 
Participants ages 16 and 17 had ACE scores 1.22 times higher than that of participants ages 14 and 15 (69 versus 56.5); however, the only score of 10 ACEs was from a participant aged 14 . Having noted this age disparity, the researcher also examined differences of means by race and sexual orientation, with results in Table 4.12. Given the small sample size, statistically significant differences in means were unlikely to be found; however, Mann-Whitney U-Tests were conducted as an additional measure. In both cases, no significant differences were found.

Table 4.12: Mean Scores: ACEs

\begin{tabular}{|l}
\hline \begin{tabular}{|l|l|l|l|}
\hline ACEs Totals by Race \\
\hline Race & $\mathrm{N}$ & Mean & $\begin{array}{l}\text { Std. } \\
\text { Deviation }\end{array}$ \\
\hline White & 4 & 5.250 & 2.9861 \\
\hline Non-White & 19 & 5.105 & 2.8654 \\
\hline
\end{tabular} \\
\begin{tabular}{l|l|l|l|}
\hline ACEs Totals by Sexual Orientation \\
\hline $\begin{array}{l}\text { Sexual } \\
\text { Orientation }\end{array}$ & $\mathrm{N}$ & Mean & $\begin{array}{l}\text { Std. } \\
\text { Deviation }\end{array}$ \\
\hline Straight & 14 & 4.357 & 2.7626 \\
\hline All Else & 8 & 6.500 & 2.7255 \\
\hline
\end{tabular}
\end{tabular}

\subsubsection{Results: Trauma Symptoms}

The second component of the YDS survey included a modified version of the Child PTSD Symptom Scale. Using the full 17-item scale, which has a possible total score of $51,{ }^{78}$ the presence of clinical PTSD is indicated by a score of 12 or higher (Fitzgerald \& Shipman, n.d.). The version of this instrument utilized in YDS was modified to include only eight items (documented in Table 4.13), with a possible total score of 24. Nevertheless, over 54 percent of respondents reported a score of 12 or

\footnotetext{
${ }^{78}$ Among PTSD-indicative items.
} 
higher. It is thus likely that, had the questionnaire utilized all seventeen standard items, more respondents would have accumulated a score of 12 or higher, indicating high prevalence of at least mild levels of clinical PTSD.

Table 4.13: Trauma Symptoms

\begin{tabular}{|l}
\multicolumn{1}{c|}{$\begin{array}{c}\text { Eight Trauma Symptoms } \\
\text { (Taken directly from the questionnaire) }\end{array}$} \\
\hline $\begin{array}{l}\text { Having upsetting thoughts about a difficult event that } \\
\text { came into your head when you didn't want them to }\end{array}$ \\
\hline Having bad dreams or nightmares \\
Feeling irritable or having fits of anger \\
\hline Acting or feeling as if a difficult event was happening \\
again (hearing something or seeing a picture and feeling \\
as if you're there again) \\
Having feelings in your body when you think about or \\
hear about a certain event (for example, breaking into a \\
sweat, heart beating fast) \\
Trying not to think about, talk about, or have feelings \\
about a difficult event \\
\hline $\begin{array}{l}\text { Having trouble concentrating (for example, losing track } \\
\text { of a story on the television, forgetting what you read, } \\
\text { not paying attention in class) }\end{array}$ \\
\hline Not being able to have strong feelings (for example, not \\
being able to cry or to feel happy)
\end{tabular}

\subsubsection{Results: Resilience}

The third component of the survey for YDS study participants included an abbreviated version of the Devereux Adult Resilience Survey (Ball \& Mackrain, 2008). Its items are documented in Table 4.14; for each one, possible responses included "Not Yet," "Sometimes," and "Almost always." To analyze the data, the researcher gave these possible responses scores of 1,2 , or 3 (respectively). All but one of the resilience items"I communicate effectively with those around me"—resulted in a mean score over 2.0. These results indicate that most participants identify at least sometimes with most of the listed aspects of resilience, and that effective communication is a recognized need or 
difficulty. This aligns with findings from the arts-based data, which frequently referred to confidence, perseverance, and resilience, yet which also indicated a sense of being unheard or misunderstood.

Table 4.14: Means for Resilience

\begin{tabular}{|c|c|c|}
\hline Resilience Item & $\mathbf{N}$ & Mean \\
\hline I am creative. & 23 & 2.26 \\
\hline I provide support to others. & 23 & 2.35 \\
\hline I express my emotions. & 22 & 2.32 \\
\hline $\begin{array}{l}\text { I try many different ways to } \\
\text { solve a problem. }\end{array}$ & 22 & 2.00 \\
\hline $\begin{array}{l}\text { I communicate effectively } \\
\text { with people around me. }\end{array}$ & 22 & 1.95 \\
\hline I am hopeful about the future. & 22 & 2.27 \\
\hline I am open to new ideas. & 22 & 2.50 \\
\hline I laugh often. & 22 & 2.27 \\
\hline I can ask for help. & 22 & 2.27 \\
\hline I am lovable. & 22 & 2.45 \\
\hline I have personal strengths. & 22 & 2.45 \\
\hline I can calm myself down. & 21 & 2.10 \\
\hline I have strong beliefs. & 21 & 2.43 \\
\hline
\end{tabular}

The Devereux Resilience Scale is typically divided into four categories:

Relationships, Internal Beliefs, Initiative, and Self-Control. To render this instrument more applicable to individuals experiencing incarceration, and to ensure minimal time demands for survey completion, this survey's modified resilience instrument utilized five of the six "Internal Beliefs" items, five of the eight "Initiative" items, and two of the four "Self-Control" items. Table 4.15 documents the mean respondent scores for each of the four categories, as well as for total resilience. Initiative and Self-Control figure here as opportunities for further development among this population. 
Table 4.15: Resilience

\begin{tabular}{|lcc|}
\hline Resilience Category & N & Mean \\
\hline Internal Beliefs & 21 & 2.3714 \\
\hline Initiative & 22 & 2.2000 \\
\hline Self-Control & 20 & 2.2500 \\
\hline Relationships & 23 & 2.3500 \\
\hline $\begin{array}{l}\text { Total Resilience Score } \\
\text { (all items) }\end{array}$ & 23 & 2.259 \\
\hline
\end{tabular}

\subsubsection{Females Ages 11 to 21 in Greater Louisville}

As noted in Chapter Three, secondary quantitative data for this population sample, intended to pair with contest writing, were drawn from an extensive survey administered in 2017 among middle and high school students throughout the Jefferson County Public School District in Louisville $(\mathrm{n}=1,889)$, by the University of Louisville's Youth Violence Prevention Research Center (YVPRC). A total of 894 respondents were excluded from analysis because they did not identify as female or as being within the age range of the study (11 to 21), leaving a sample of 995. The original YVPRC survey included 146 items for high school students and 132 items for middle school students; these fell into 13 sections or categories: Demographics, Extent of Exposure to Violence, Social Supports, Community Perception, Sense of Sociopolitical Control, ${ }^{79}$ Extent of Community Activity/Involvement, Resilience, Cultural Identity, Thoughts about Society, Attitudes toward Violence, Peers' Behaviors, Peers' Expectations (social norms), and Personal Violent Behaviors. For maximum relevance to the current study's research

\footnotetext{
${ }^{79}$ Sociopolitical control items were included in the high school surveys only.
} 
questions (and comparative analysis with arts-based methods), quantitative analysis of survey results incorporated variables from all but the final three categories.

\subsubsection{Demographics}

Demographic information for study participants is documented in Table 4.16:

Table 4.16: Demographics: YVPRC/Louisville

\begin{tabular}{|llcc|}
\hline & & & \\
Demographic Variable & N & Percentage \\
\hline Age & 11 & 132 & 13.3 \\
& 12 & 258 & 25.9 \\
& 13 & 254 & 25.5 \\
14 & 178 & 17.9 \\
15 & 64 & 6.4 \\
16 & 45 & 4.5 \\
17 & 45 & 4.5 \\
& 18 & 18 & 1.8 \\
Race & 1 & .1 \\
& Total & $\mathbf{9 9 5}$ & $\mathbf{1 0 0 . 0}$ \\
& White & 378 & 46.3 \\
& Black & 234 & 28.6 \\
Other & 63 & 7.7 \\
Mixed & 60 & 7.3 \\
Hispanic & 38 & 4.7 \\
Asian & 31 & 3.8 \\
American Indian / & 13 & 1.6 \\
Native American & & \\
Missing & 178 & 17.9 \\
Total & $\mathbf{9 9 5}$ & $\mathbf{1 0 0 . 0}$ \\
\hline
\end{tabular}

\subsubsection{Results: Exposure to Violence}

Notably for the current study, items in this survey's "Exposure to Violence" category included only one question regarding respondents' personal victimization ("My house has been broken into"). Remaining items inquired only about forms of violence witnessed by respondents. As a result, the extent of respondents' personal victimization cannot be ascertained from the survey data. This is in stark contrast to the arts-based data 
from Louisville girls, in which personal experiences or fears of violence comprised a significant portion of the material.

All nine exposure items from the YVPRC survey are documented in Table 4.17. Among women, the most prominent witnessed forms of violence were: seeing someone arrested (59\% had witnessed this at least once); seeing someone get beaten up (58.1\% had witnessed this at least once); and hearing gun shots (57.7\% had heard shots at least once). ${ }^{80}$

Table 4.17: Frequencies: Exposure to Violence

\begin{tabular}{|lc|c|c|c|c|}
\hline \multicolumn{1}{|c}{ Form of Violence } & Never & $\%$ & Once+ & $\%$ & Total \\
\hline I have heard guns being shot. & 417 & 42.3 & 569 & 57.7 & 986 \\
I have seen somebody get arrested. & 404 & 41 & 581 & 59 & 985 \\
I have seen drug deals go down. & 706 & 72 & 274 & 28 & 980 \\
I have seen someone getting beaten up. & 406 & 41.3 & 578 & 58.1 & 984 \\
My house has been broken into. & 773 & 78.8 & 208 & 20.9 & 981 \\
$\begin{array}{l}\text { I have seen somebody get stabbed or } \\
\text { shot. }\end{array}$ & 875 & 89.4 & 104 & 10.6 & 979 \\
I have seen a gun in my home. & 684 & 70.2 & 291 & 29.8 & 975 \\
$\begin{array}{l}\text { I have seen gangs in my neighborhood. } \\
\text { I have seen somebody pull a gun on } \\
\text { another person. }\end{array}$ & 748 & 76.1 & 235 & 23.9 & 983 \\
& & 85.5 & 142 & 14.5 & 982 \\
\hline
\end{tabular}

Given that having one's house broken into was the survey's only explicit inquiry regarding personal victimization, it is noteworthy that over 20 percent of young women reported having had this experience. In addition, one in 10 women (10.6\%) reported witnessing someone being stabbed or shot, and nearly one in six (14.5\%) reported having seen someone pull a gun on another person-both potentially traumatizing situations. These results again align with the arts-based data — and contest writing in particular. In

\footnotetext{
${ }^{80}$ Note that the survey did not specify in what context gun shots may have been heard; as a result, it is possible that young women who had heard gun shots while hunting or at a range (as opposed to in their residential neighborhood) were included in this total.
} 
that data set, guns and gun violence were prominent topics, as was personal victimization and trauma related to community violence-including seeing a friend killed on the street.

\subsubsection{Results: Community Involvement, Civic Engagement}

The YVPRC survey began with questions about respondents' perceptions of their roles in their communities, ${ }^{81}$ which provided insights into how young women engaged with their immediate contexts. For example, 77.2 percent of young women in high school indicated that they agreed that it is their "responsibility to help improve the community," with 76.3 percent agreeing that they "feel a personal obligation to contribute in some way to the community," and 71.7 percent feeling that they "have the power to make a difference in the community." 82 Notably, young women in middle school showed far higher rates of personal obligation and potential influence; for example, nearly all middle school respondents (96.6\%) agreed that "Helping other people is something everyone should do, including myself," and four out of five (81.2\%) reported feeling like they "can make a difference in the community." These age disparities suggest the need for further research regarding decreasing rates of optimism, efficacy, and involvement over time. Apart from age disparities (which were not evaluated for the current study), these findings echo results from the writing contest and all arts-based data sets, in which

\footnotetext{
${ }^{81}$ Sixteen items were included on the high school version; 10 items were included on the middle school version.

${ }^{82}$ However, less than half of high school respondents (47.9\%) reported that they currently "participate in political or social causes in order to improve the community." The gap between the value respondents place on responsibility and the level of their actual action may indicate a need for improved education regarding civic engagement, as well as expanded opportunities for youth contributions. Notably, given that the current study is invested in methodological considerations (including linguistic and cultural influences), such a gap may also indicate the need for additional response options regarding how young women apply their values to their lived experience. For example, young women may be actively working to reduce violence, but do not recognize or define these actions as means of participating in "political" or social "causes."
} 
Caring for Others and Encouraging/Advising Others were significant themes, as were the needs to ACT and to Speak Out.

Along similar lines, the high school version of this survey included eight "Sociopolitical Control" items to assess the level to which youth perceived themselves as having power or influence regarding community outcomes and decisions. ${ }^{83}$ When these were totaled to create a single Sociopolitical Control variable, 124 women $(55.1 \%)$ fell within the overall "Agree" range, with a mean score of 3.99 for social control (on a scale of one to six: Strongly disagree to Strongly agree). These findings indicate a youth asset: over half of women-identified youth felt a sense of efficacy with regard to improving their communities. However, these findings simultaneously indicate a significant need or barrier to health and safety—in that 44.9 percent of young women reported that they did not perceive they should or can be meaningfully involved in political or social issues affecting their communities.

A limitation of the YVPRC survey is the inability to ascertain why young women felt unable or disinclined to be further involved in their community; by contrast, the corresponding contest writing data set provided potential reasons. Although writers did not indicate feeling that they should not be involved in political or social issues (typically, they indicated the opposite), they did indicate hopelessness and overwhelm, which can lead to failures to engage. Writers also regularly indicated the felt sense of being unheard, unnoticed, or disbelieved; further research should investigate the extent to which this perception is connected to levels of civic (dis)engagement.

\footnotetext{
${ }^{83}$ These items, which were not included in middle school surveys, included statements such as "I believe that I can have enough influence to impact community decisions," or "I try to find time or a way to make a positive difference in the community."
} 


\subsubsection{Results: Attitudes toward Violence}

The YVPRC survey also asked about youths' beliefs and attitudes toward violence. The majority of young women $(86.2 \%)$ agreed that "It is possible to reduce youth violence" and that "Reducing youth violence is important" to them. Three-quarters of young women $(75.8 \%)$ reported that they were "likely to do something in the efforts to reduce youth violence." Clearly, young women's explicit interest in reducing youth violence suggests an asset; these particular statements also imply hope as an additional potential asset among respondents. Both assets echo contest writing, in which young women clearly felt that reducing violence was important to them, and indicated hope for the future. Meanwhile, over one in five young women (22.6\%) expressed disinclination to help reduce youth violence, which - similar to the community engagement issue above-warrants further research.

All remaining items in the "Attitudes toward Violence" category were aggregated into three new variables: Accepting of violence, Violence as necessary (indicating the sense that violence may be required under certain circumstances ${ }^{84}$ ), and Opposition to violence. Nearly one-quarter of the respondents (23.4\%) agreed or strongly agreed with items associated with accepting violence, and over one-third (36.8\%) agreed or strongly agreed with items associated with obligated violence. However, the vast majority of young women $(83.2 \%)$ agreed or strongly agreed with items associated with non- or antiviolent attitudes, such as "There are better ways to solve most problems than by fighting."

These items offered insights that contrast with the arts-based data (and contest writing in particular), in which writers did not indicate acceptance of violence, and

\footnotetext{
${ }^{84}$ For example, "Sometimes you have only two choices: get punched or punch the other person first."
} 
obligated violence was indicated only implicitly. ${ }^{85}$ A potential limitation of the writing contest data is the premise of the contest itself, which involved selection bias toward individuals with anti-violence beliefs. On the other hand, the contest data did of course consistently refer to the existence and prevalence of violent individuals in writers' lives and varied contexts. This cannot compensate for an inability to hear directly from such individuals, nor does it equate to findings from the YVPRC survey. However, it is noteworthy that individuals who do not themselves participate in creative writing are often personally and descriptively brought into the data.

\subsubsection{Results: Social Supports}

The YVPRC survey also included an assessment of youths' social supports. When asked the extent to which they agreed with the statement, "There are people I can depend on to help me if I really need it," just 5.6 percent indicated that they disagreed $(93.3 \%$ agreed). However, when the question was phrased in a different way, the results shifted: $12.2 \%$ indicated they agreed with the statement, "There is no one I can depend on for help if I really need it." The latter statement, though similar to the former, resulted in over twice as many respondents indicating a lack of (and thus a need for) social supports. This finding is relevant to the methodological analysis at hand, as it highlights the potential influence on data results of linguistic construction and emotional resonance. Meanwhile, given that most respondents $(87.9 \%)$ indicated disagreement with the latter statement, it

\footnotetext{
${ }^{85}$ For example, one writer noted, "My mom would get depressed, drunk, and start fights...she pushed me down the stairs and threw bleach on me. Me and Juan drug her through the alley one time because she started yelling and cussing us out and started swinging." This writer did not explicitly state that violence may at times be necessary; however, her writing indicates that she may have perceived her and Juan's actions as necessary during the described experience.
} 
is clear that most young women do have some measure of social supports in their lives; this is a potential asset in improving health and safety.

\subsubsection{Results: Mann-Whitney U Tests}

Having already noted grade-level disparities in community involvement and youth violence beliefs (middle school versus high school), the researcher tested assumptions for Mann-Whitney U tests as a precursor for examining grade-level differences in beliefs about reducing youth violence, perceived presence of social supports, and exposure to violence. Only "Beliefs about Reducing Youth Violence" satisfied the assumption of similar distributions; the resulting test showed that such beliefs were significantly more positive for middle school respondents than for high school students $(U=68671.5, p=.000)$. This reinforces other YVPRC findings indicating greater engagement and optimism among middle school women.

\subsubsection{Results: Kruskal-Wallis H Tests}

The researcher also tested assumptions for Kruskal-Wallis $\mathrm{H}$ tests, to examine differences by race in these same variables (beliefs about reducing youth violence, perceived presence of social supports, and exposure to violence). Only the first two satisfied the assumption of similar distributions, and the resulting tests (see Table 18) showed that there was no statistically significant difference by race in young women's Beliefs about Reducing Youth Violence $(\mathrm{H}(2)=11.742, p=.068)$. However, there was a statistically significant difference by race in young women's perception of social supports $(\mathrm{H}(2)=13.560, p=.035)$, with white respondents reporting higher levels of perceived social support than non-white respondents. Mean ranks are listed in Table 4.18. 
Table 4.18: Kruskal-Wallis Tests

Ranks

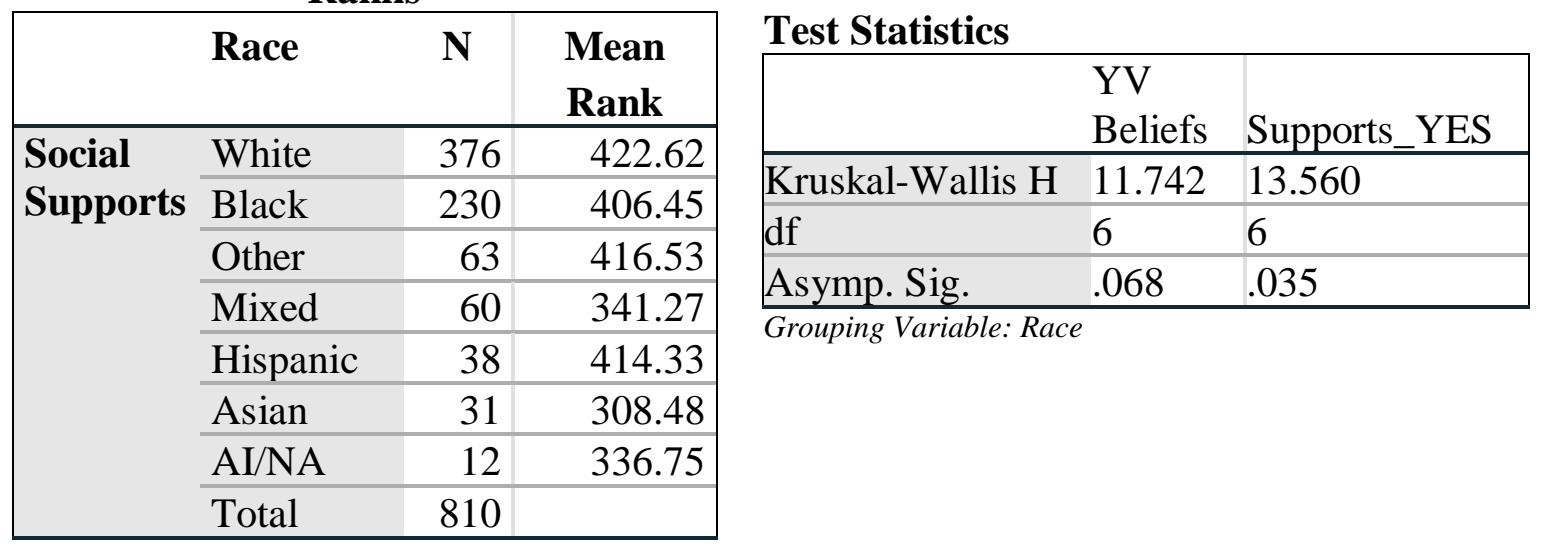

Uncovering such differences is a significant affordance of survey data-which can quantify concerns such as the lower rate of perceived social supports among minority women. In contest data, racial disparities arose via content - such as when young women wrote about experiences of oppression based on race. Given that writers provided demographic data when entering the contest, it may be possible quantify the contest data by identifying themes, disaggregating entries by race, and then further analyzing frequency counts. Regardless, both data sets indicate the need for increased cultural- and gender-responsiveness in schools and other youth-serving institutions, in which a young woman's sense of support may be precluded by her exclusion from dominant cultures or norms.

\subsubsection{Results: Spearman Rand Order Correlation Tests}

Finally, the researcher created scatterplot matrices to test for monotonicity among several variables in the YVPRC survey, as a precursor to running Spearman Rand Order Correlation tests. Only [Exposure to Violence + Perceived Presence of Social Supports] and [Exposure to Violence + Beliefs about Reducing Youth Violence] indicated monotonicity, and the resulting Spearman Rand tests showed that both of these had weak 
negative relationships (see Table 4.19). Higher social supports scores were weakly correlated with less violence exposure, and higher ratings of the importance and possibility of reducing youth violence were weakly correlated with less violence exposure. Further research will need to be conducted to discern how these variables are related.

Table 4.19: Violence Exposure, Social Supports, Beliefs Exposure to violence + Perception of Available Social Supports

\begin{tabular}{|lllll|}
\hline & & & \multicolumn{3}{c|}{$\begin{array}{l}\text { Social } \\
\text { Supports }\end{array}$} & Exposure \\
\hline Spearman's rho & Social & Correlation Coefficient & 1.000 & $-.146^{* *}$ \\
& Supports & Sig. (2-tailed) &. & .000 \\
\cline { 3 - 5 } & & $\mathrm{N}$ & 985 & 980 \\
\cline { 3 - 5 } & Exposure & Correlation Coefficient & $-.146^{* *}$ & 1.000 \\
\cline { 3 - 5 } & & Sig. (2-tailed) & .000 &. \\
\cline { 3 - 5 } & & 980 & 987 \\
\hline ** Correlation is significant at the 0.01 level (2-tailed) & & \\
\hline
\end{tabular}

Exposure to violence + Belief that reducing youth violence is important and possible

\begin{tabular}{|lllll|}
\hline & & & Exposure & YV_Beliefs \\
\hline \begin{tabular}{llll|} 
Spearman's \\
rho
\end{tabular} & Exposure & Correlation Coefficient & 1.000 & $-.187^{* *}$ \\
\cline { 3 - 4 } & & Sig. (2-tailed) &. & .000 \\
\cline { 2 - 4 } & & $\mathrm{N}$ & 987 & 978 \\
\cline { 2 - 4 } & YV_BeliefCorrelation Coefficient & $-.187^{* *}$ & 1.000 \\
\cline { 3 - 4 } & $\mathrm{s}$ & Sig. (2-tailed) & .000 &. \\
\cline { 3 - 5 } & & $\mathrm{N}$ & 978 & 985 \\
\hline
\end{tabular}

**. Correlation is significant at the 0.01 level (2-tailed).

\subsection{3: Female Students at the University of Louisville}

As noted in Chapter Three, arts-based data for this population were paired with two University of Louis ville surveys: "CardSafe" and "Well-Being and Resilience." Results from analyses of these surveys are presented separately below. 


\subsubsection{CardSafe Survey: Introduction}

The CardSafe survey was administered to students at the University of Louisville in Spring 2016 by the university's Office of Institutional Effectiveness $(n=613)$. One hundred forty-six respondents were excluded from analysis either because they did not identify as female or because they did not identify as undergraduate students; $; 6$ this left a sample of 376 . The CardSafe survey included 247 total items, differentially available to each respondent via the use of skip logic. The survey measured respondent perceptions, experiences, and behaviors related to violence, safety, health, sex and relationships, and access to resources.

\subsubsection{CardSafe Demographics}

Although this survey did not collect information regarding race or age, other demographic information for participants in this study is documented in Table 4.20.

Table 4.20: Demographics: CardSafe Survey

\begin{tabular}{|llcc|}
\hline $\begin{array}{l}\text { Demographic } \\
\text { Variable }\end{array}$ & & N & Percentage \\
\hline Year & Freshman & 82 & 21.8 \\
& Sophomore & 80 & 21.3 \\
& Junior & 102 & 27.1 \\
& Senior & 112 & 29.8 \\
Sexual & Total & $\mathbf{3 7 6}$ & $\mathbf{1 0 0 . 0}$ \\
Attraction & & & \\
& Only attracted to males & 278 & 75.1 \\
& Mostly attracted to & & \\
& males & 64 & 17.3
\end{tabular}

\footnotetext{
${ }^{86}$ As noted in Chapter 3, this survey did not inquire about respondents' age. To best achieve similarity to this study's target population (ages 11-21), analysis of CardSafe data included only those respondents who identified as being a Freshman, Sophomore, Junior, or Senior - and were thus more likely to be age 21 or under. It is possible that many "nontraditional" students beyond the age range for this study completed the CardSafe survey.
} 
Equally attracted to males and females Mostly attracted to females

Only attracted to

females

Not sure

Missing

Total

$\begin{array}{cc}17 & 4.6 \\ 5 & 1.4 \\ & \\ 5 & 1.4 \\ 1 & 0.3 \\ 6 & \\ \mathbf{3 7 6} & \mathbf{1 0 0 . 0}\end{array}$

\subsubsection{CardSafe Results: Experiences of Violence}

The CardSafe survey inquired about several forms of violence that respondents may have personally experienced in the previous twelve months; these are documented in part in Table 4.21. Most prevalent were experiences of sexual harassment, bullying, and verbal abuse. The prevalence of sexual harassment reflects Wildfire writing, in which sexual assault/harassment was one of the top four themes.

Table 4.21: Personal Experiences of Violence

\begin{tabular}{|c|c|c|c|c|}
\hline \multirow{5}{*}{$\begin{array}{l}\text { Not a dating } \\
\text { or long-term } \\
\text { partner }\end{array}$} & Form of Violence & $\begin{array}{c}\text { Separate } \\
\text { Indications }\end{array}$ & Min. $\mathbf{N}$ & Percentage \\
\hline & \multirow{4}{*}{$\begin{array}{l}\text { Sexual harassment } \\
\text { Bullying } \\
\text { Verbal abuse } \\
\text { Physical abuse/harm } \\
\end{array}$} & 236 & 90 & 24.1 \\
\hline & & 168 & 93 & 15.4 \\
\hline & & 67 & 60 & 16.1 \\
\hline & & 25 & 14 & 3.8 \\
\hline \multirow{5}{*}{$\begin{array}{c}\text { Dating or } \\
\text { long-term } \\
\text { partner }\end{array}$} & \multirow{5}{*}{$\begin{array}{l}\text { Stalking } \\
\text { Monitoring; invasion } \\
\text { of privacy } \\
\text { Threatening behaviors } \\
\text { Bullied, mistreated in } \\
\text { front of others } \\
\text { Physical abuse/harm }\end{array}$} & 89 & 28 & 7.5 \\
\hline & & 32 & 32 & 12.6 \\
\hline & & 31 & 21 & 8.2 \\
\hline & & 28 & 28 & 11 \\
\hline & & 27 & 10 & 4 \\
\hline Either & Unwanted sex & 24 & 24 & 6.5 \\
\hline
\end{tabular}


Twenty-four female respondents $(6.5 \%)$ reported that they had had unwanted sex at least once within the last 12 months; of those 24, two indicated that the experience had resulted in physical injuries, and six reported having subsequent problems meeting school obligations. Thirteen women $(59.1 \%)$ reported "serious emotional difficulties" following this experience. These data again align with the focus in Wildfire on sexual assault and rape and the lingering traumatic effects that result. Both data sets indicate the need for further education regarding relationships, consent, and communication; ${ }^{87}$ they also indicate the need for ready access to resources for individuals experiencing relationship concerns or violence.

\subsubsection{CardSafe Results: Witnessing Violence}

The CardSafe survey also inquired about forms of threat or violence that respondents may have witnessed or suspected in the previous 12 months; these are documented in part in Table 4.22.

\footnotetext{
${ }^{87}$ Further research should assess the priority population for such education; for example, although the current study is focused on women's experiences and perceptions of violence, it may be that education for and among males would be the most effective priority target for responsive resources.
} 
Table 4.22: Witnessed/Suspected Violence

\begin{tabular}{|c|c|c|}
\hline Form of Violence & $\mathbf{N}$ & Percentage \\
\hline $\begin{array}{l}\text { Suspected or knew another } \\
\text { student was experiencing } \\
\text { intimate partner violence }\end{array}$ & 60 & 16.1 \\
\hline $\begin{array}{l}\text { Suspected or knew that } \\
\text { someone was being led } \\
\text { away for sex while unable } \\
\text { to consent }\end{array}$ & 59 & 16.2 \\
\hline $\begin{array}{l}\text { Suspected or knew that } \\
\text { another student's drink was } \\
\text { being drugged }\end{array}$ & 54 & 14.7 \\
\hline $\begin{array}{l}\text { Heard another student } \\
\text { bragging/joking about } \\
\text { coercing someone into sex }\end{array}$ & 52 & 14.1 \\
\hline
\end{tabular}

One in six women $(16.1 \%)$ reported suspecting or knowing at least once that another student was experiencing intimate partner violence; a similar number $(16.2 \%)$ reported suspecting or knowing at least once that someone was being led away for sex while unable to consent. Similarly, one in seven women reported having heard someone joke or brag about "forcing another student to have sex with them" (14.1\%), and one in seven also reported suspecting or knowing (at least once) that another student's drink was being drugged $(14.7 \%)$. While these numbers appear relatively low, they indicate a looming significant threat faced by young women - the presence and influence of which is reinforced by the finding that 88.2 percent had, in the previous 12 months, avoided places "where alcohol or drugs might lead to problems." The threat of such experiences has 
health implications (Rosenberg, 2017; Ropeik, 2004), and is echoed by the prominence of Fear/Threat as its own form of violence in the arts-based data.

\subsubsection{CardSafe Results: Personal Response/Action}

The CardSafe survey also asked about typical perceptions of or responses to violence. The vast majority of respondents $(88.5 \%)$ indicated that they would personally try to change a situation in which a student is being hurt. Nearly all women $(98.3 \%)$ indicated that they would "Respect someone who did something to stop or prevent the behavior." This echoes arts-based findings regarding the need to ACT and the violence of inaction. However, three-quarters of female respondents (73\%) indicated that, if others were present, they would let someone else step in to help the person being hurt. This apparent disparity between personal values and expected personal behavior may indicate the need for bystander training; it may also indicate fear or threat associated with intervention - and the perception that others could perhaps carry out such action with less danger to themselves.

Notably, the phrasing of these questions highlights some linguistic and cultural limitations of the survey format. While the broad phrasing of the question- -If I saw a student hurting another student, I would intervene..."-may in some sense be useful, it privileges those forms of violence that are most blatant or public. For example, is knowing that a student is abusing their partner in their home the same as "seeing a student hurt another student"? The survey may therefore fail to measure students' willingness to help when the "hurt" experienced by another student is perceived as a private relational matter. This conundrum was reflected in the Wildfire writer's urgent call for their audience members to "knock on that door" when they hear couples fighting. 
Communicative limitations were also apparent in CardSafe questions about whether students had "received information or attended a program about" bystander training (32.6\% indicated yes), how to report sexual assault (38.8\% indicated yes), or how to help victims of sexual assault (51.6\% indicated yes). Actively attending a program — when not mandatory—can indicate active interest in or a sense of responsibility toward reducing violence; however, this survey item combined active interest with the passive receipt of information. As a result, it was impossible to analyze the extent to which young women at the university may have been actively interested in these or similar opportunities. ${ }^{88}$

\subsubsection{Well-Being and Resilience Survey: Introduction}

The Well-Being and Resilience (WBR) survey was administered to students at the University of Louisville in Spring 2018 by the university's Office of Health Promotion (n $=1,327)$. A total of 860 respondents have been excluded from analysis either because they did not identify as female or because they did not identify as being within the study's target age range (11-21), yielding a sample of 467. The WBR survey included 101 items, including four open response questions ${ }^{89}$ and it measured a range of respondent behaviors and beliefs related to health, well-being, safety, and access to resources.

\footnotetext{
${ }^{88}$ This survey item does allow the university to assess the extent to which distributed information has reached students (and been recalled by them) — which may have been its primary intent.

${ }^{89}$ While full analysis of the open response questions is beyond the scope of this study, it is worth including a brief note about the first: "What additional life challenges (not included in previous questions) are currently barriers to your academic success and/or wellbeing?" Anxiety and overwhelm figured prominently in open responses. Anxiety was mentioned only twice by name; however, both concerns were frequently referenced indirectly or via symptom descriptions (e.g., "there's a lot on my plate," "Too many obligations," "self-doubt," "work stress," "Uncertainty about future employment," etcetera). These responses indicate the potential need for additional survey questions regarding anxiety and mental health. They also indicate that respondents may not resonate with the term "anxiety;" future surveys may therefore need to find creative ways to assess mental health without restricting inquiries to this term.
} 


\subsubsection{WBR Demographics}

Demographic information for participants in this study is documented in Table 4.23.

Table 4.23: Demographics: WBR

\begin{tabular}{|llcc|}
\hline Demographic & N & Percentage \\
\hline Variable & 89 & 19.10 \\
Age & 18 & 159 & 34.00 \\
19 & 124 & 26.60 \\
20 & 95 & 20.30 \\
21 & $\mathbf{4 6 7}$ & $\mathbf{1 0 0 . 0 0}$ \\
Total & & \\
Race & White & 337 & 72.20 \\
& Black/African & 46 & 9.90 \\
American & 31 & 6.60 \\
Asian & 22 & 4.70 \\
Hispanic/Latino & & \\
Native & 1 & 0.20 \\
Hawaiian/Other & & \\
Pacific Islander & & \\
Two or More & 30 & 6.40 \\
Races & & \\
Total & $\mathbf{4 6 7}$ & $\mathbf{1 0 0 . 0 0}$ \\
Sexual Orientation & & \\
Straight/Hetero & 369 & 79.00 \\
Bisexual & 51 & 10.90 \\
Asexual & 14 & 3.00 \\
Pansexual & 11 & 2.40 \\
Lesbian & 6 & 1.30 \\
Queer & 6 & 1.30 \\
Other & 5 & 1.10 \\
Gay & 1 & 0.20 \\
Missing & 4 & 0.90 \\
Total & 467 & 100.00 \\
\hline
\end{tabular}




\subsubsection{WBR Results: Experiences of Violence and Safety}

When asked how safe they felt in their daily lives, 6.6 percent reported feeling "not at all" or only "slightly" safe, while 30.3 percent reported feeling moderately safe. Nearly two-thirds of the women responding to the survey $(63.1 \%)$ reported feeling "very much" or "extremely" safe. While predominantly positive, these findings do reflect the common experience of fear/threat illuminated in the arts-based data. However, unlike the arts-based data, these findings do not immediately indicate potential causes for perceived safety or its absence.

When asked if they knew "another UofL student who has been directly impacted by sexual or relational violence," over 40 percent of women indicated that they did know such a student — which notably is greater than the proportion indicating that they definitely did not (36.6\%). ${ }^{90}$ Similarly, students were asked if they knew "another individual who has experienced suicidal thoughts or behavior." While this item is less immediately indicative of respondents' personal sense of safety, it does illuminate safety concerns overall—including the prevalence of suicidality and the need for responsive resources. Over two-thirds of female respondents (67.7\%) reported knowing someone who had experienced suicidal thoughts or behavior. These findings again echo arts-based data regarding sexual assault/harassment, rape, mental health concerns, and suicide/selfharm.

\subsubsection{WBR Results: Needs and Barriers}

Corresponding with findings about suicidality, nearly one in five women (17.8\%) reported feeling hopeless most or all of the time. Given that the presence of hopelessness

\footnotetext{
90 The remaining respondents, 104 women $(22.4 \%)$, indicated that they were not sure whether they knew such a student.
} 
is more indicative of a potential risk for suicidality than the lack of hope (Huen et al., 2015), and given that two-thirds of respondents had indicated knowing someone who had experienced suicidality, these findings suggest a significant safety need echoing hopelessness/overwhelm in the arts-based data.

Table 4.24: Frequency with which women obtained verbal consent

\begin{tabular}{|lcc|}
\hline Frequency & N & Percentage \\
\hline Never & 33 & 7.2 \\
Rarely & 10 & 2.2 \\
Sometimes & 25 & 5.5 \\
Often & 59 & 12.9 \\
Always & 331 & 72.3 \\
Total & 458 & 100.0 \\
Missing & 9 & \\
Total & 467 & \\
\hline
\end{tabular}

Another safety-related item asked how often students obtained verbal consent in their "romantic or sexual experiences," documented in Table 4.24. Responses suggest a need for further education and resources that help women feel confident asking for (and being asked for) consent. They also align with prominence of consent as an issue in the Wildfire play.

Nearly one in three women (32.1\%) agreed or strongly agreed that they have "a hard time making it through stressful events." ${ }^{91}$ A similar proportion (32.4\%) indicated that their social relationships were not supportive and rewarding, and one in 10 women reported dissatisfaction with their family lives. ${ }^{92}$ Extending the concept of social supports

\footnotetext{
${ }^{91}$ A larger proportion of women (201, or 43.3\%) reported disagreeing or strongly disagreeing with this statement, indicating resilience and a potential asset among young women.

${ }^{92}$ On a scale of one to ten (one being "Very dissatisfied" and ten being "Very Satisfied"), thirteen percent of women selected four or below.
} 
to a sense of community, nearly one in six women $(15.4 \%)$ indicated that in the previous month, they did not feel part of a community even once. ${ }^{93}$ Further research may explore possible correlations between difficulty during stressful times and lack of social supports. For example, if the relationship is not causal (lack of social supports causing increased difficulty), it is likely to at least be mutually reinforcing — in that stress difficulties combined with a perceived lack of support may lead to worse health outcomes than stress difficulties on their own.

Perhaps contributing to stress, nearly one in four women $(24.8 \%)$ reported being "not at all" or only "a little" able to accept their bodily appearance. On the individual level, this finding indicates the need for further means of increasing women's confidence and health; it also represents a barrier to individual well-being and a potential barrier to individual safety. Beyond the individual, this finding echoes arts-based findings regarding structural violence-because it points to the effects of sexism, gender roles/expectations, and the related, ongoing importance of beauty (even when variously defined) to women's felt sense of success or well-being.

The WBR survey also asked students how they would rank their health on a scale from one to five (Poor to Excellent). For all women, the mean self-report score was 3.17, with 23.2\% selecting "Poor" or "Fair" to describe their health. To illuminate potential disparities, the researcher ran Mann Whitney U-tests to examine differences in selfreported health scores by race and sexual orientation. Results indicated that scores were not significantly different for white and non-white students; however, scores were

\footnotetext{
${ }^{93}$ As noted throughout, indications of needs and barriers inevitably exist alongside indications of assets. For example, most women (60.4\%) reported having felt at least once a week like they were part of a community. This illustrates women's abilities to forge and sustain community - a significant asset that could perhaps inform the need among other women for increased social supports.
} 
significantly higher for heterosexual women than for non-heterosexual women $(U=$ $12340, p=<.001)$. The mean health score for heterosexual women was 3.28 , compared to 2.78 for non-heterosexual women—suggesting that sexual orientation affects female students' perception of their health. Means for each identified sexual orientation are documented in Table 4.25 .

In addition to asking students to rank their health, the WBR survey also asked them to rank their satisfaction with their access to health care on a scale from one to five.

Table 4.25: Self-Reported Health by Sexual Orientation

\begin{tabular}{|l|l|l|l|}
\hline Sexual Orientation & Mean & N & $\begin{array}{l}\text { Std. } \\
\text { Dev. }\end{array}$ \\
\hline Straight/Heterosexual & 3.28 & 369 & 0.924 \\
\hline Bisexual & 2.69 & 51 & 0.812 \\
\hline Asexual & 2.93 & 14 & 0.997 \\
\hline Pansexual & 3.09 & 11 & 0.539 \\
\hline Lesbian & 2.83 & 6 & 0.983 \\
\hline Queer & 2.67 & 6 & 0.516 \\
\hline Other & 2.6 & 5 & 1.342 \\
\hline Gay & 3 & 1 &. \\
\hline Missing & 3.5 & 2 & 0.707 \\
\hline Total & $\mathbf{3 . 1 8}$ & $\mathbf{4 6 5}$ & $\mathbf{0 . 9 2 6}$ \\
\hline
\end{tabular}

Women's mean response was $4.17(S D=.954)$, suggesting that access to health care is an asset among women who are attending the university. However, given that nearly one in four women (23.9\%) reported a lack of satisfaction with their access, this finding also suggests a need for improvement. A Mann-Whitney U test also showed a significant difference in health care satisfaction between heterosexual and non-heterosexual women 
$(\mathrm{U}=20399, p=.003)$. The mean satisfaction rating for heterosexual women was 4.1, compared to 3.71 for non-heterosexual women.

Aligning with the potential for identity to influence health, the WBR survey also inquired whether students' personal identity was "valued at UofL." While 43.5 percent selected "Yes," nearly one-third (31.9\%) selected "No" or "I don't know." This again suggests a need for more inclusive health care options among minority women, including clearer, more frequent, or more identity-responsive communications and resources. No significant differences were found for this variable by race. While arts-based data often illuminated health concerns, which ranged from broad awareness to individual health narratives, it was not able to deliver concise self-reports of health such as these, nor was it able to identify disparities according to race or sexual orientation.

Finally, the WBR survey presented students with 15 common health issues, asking whether any of these items had affected the respondent's academic status in the previous 12 months. Among young women, stress was the most common response (72.8\%), followed by anxiety (51.6\%), relationships (37.7\%), and depression (36.4\%).

All included issues and their frequencies are documented in Table 4.26.

Table 4.26: Frequency of Issues Affecting Women's Academic Status

\begin{tabular}{|l|cc|}
\hline \multicolumn{1}{|c|}{ Issue } & N & Percentage \\
\hline Access to healthcare & 21 & 4.5 \\
Stress & 340 & 72.8 \\
Alcohol or drug use & 31 & 6.6 \\
Relationships & 176 & 37.7 \\
Depression & 170 & 36.4 \\
Anxiety & 241 & 51.6 \\
Fear & 80 & 17.1 \\
Illness & 76 & 16.3
\end{tabular}




\begin{tabular}{|l|cc|} 
Worry about other people & 115 & 24.6 \\
Discrimination/bullying & 8 & 1.71 \\
Pain & 34 & 7.3 \\
Trauma & 21 & 4.5 \\
Suicidal thoughts & 47 & 10.1 \\
Self-harm & 21 & 4.5 \\
Loss of someone & & \\
close to you & 60 & 12.9 \\
\hline
\end{tabular}

\subsubsection{WBR Results: Assets, Attributes, and Values}

A range of items on the WBR survey elicited information regarding respondents' values and perceptions, allowing an assessment of assets as well as potential needs and barriers. For example, when asked how much they agreed with the statement "I lead a purposeful and meaningful life," 80 percent of young women indicated agreement. This is a clear health asset among these women; however, this finding also indicates that one in five women was unable to agree that she lived a purposeful and meaningful life. This suggests a need among young women for increased opportunities not only for general achievement, but specifically for meaning-making and belonging. Notably, arts-based data indicated these needs (and assets) as well, with some writers finding meaning in the act of writing itself. ${ }^{94}$

Continuing with self-perception and assets, nine out of 10 women (90.2\%) agreed that they "actively contribute to the happiness and well-being of others;" slightly fewer (88.5\%) agreed that they are "a good person and live a good life." Similarly, over nine in 10 women (91.4\%) agreed that they are "competent and capable in the activities that are important" to them. These findings again echo assets and values themes from the arts-

\footnotetext{
${ }^{94}$ This may be particularly true for the writers of How to Start a Wildfire, who provided indications throughout the play that telling their story and (thereby) helping other survivors provided a sense of purpose that became part of their healing.
} 
based data, including caring for others, confidencelempowerment, encouraging/advising others, and pride. In both the WBR and arts-based data sets, such findings suggest significant assets for young women's health and well-being. Notably, given that one in 10 women were unable to agree that they contribute to others' well-being, that they are good, or that they are competent, the survey findings also echo violence and barrier themes in the arts-based data, such as insecurity, self-worth, and no one notices. In both the quantitative and qualitative forms of data, confidence, meaning, and meaningful connection emerge as important needs for women's well-being.

Fewer women—but still a majority (83.4\%)—agreed that they were "optimistic about my future," and nearly two-thirds of women (63.5\%) strongly identified with the statement, "I have overcome setbacks to conquer an important challenge," with another 30 percent identifying with it "somewhat." These results reveal significant assets in women's confidence and optimism regarding their lives, work, and futures. They also reveal barriers, in that many women found themselves unable to agree or identify with these statements. These assets and barriers are both reflective of prominent codes in the writing data sets.

\subsection{Summary of Findings Regarding Violence}

Overall results from analyses of qualitative and quantitative data indicate the ubiquity of experiences of violence among young women in Louisville, and the need for services and practices designed to hear and respond to young women's accounts. Young women reported high rates of experiencing or witnessing: sexual assault (or its attempts), domestic and intimate partner violence, and bullying. Survey data also revealed high rates of: stress, anxiety, general mental health concerns, and knowing friends or peers who had 
experienced suicide ideation. Across the three arts-based data sets, young women's most reported concerns or experiences with interpersonal violence were domestic and intimate partner violence, trauma, and fear or threat. They also frequently discussed addiction, gun violence, grief or loss, bullying, sexual assault, and child abuse. Writers' depictions of these issues included references to structural issues such as institutional inaction, gender roles, mass shootings, and incarceration — which largely failed to appear in the surveys.

The data also indicate that young women commonly experienced isolation, loneliness, disconnection, dismissal or disregard, and a sense of having no social supports. While these factors are typically figured as barriers to safety and health, as causes of behavioral concerns, or as results or exacerbators of trauma, the arts-based data portrayed them as direct sources of harm — and thus as forms of violence in their own right. In fact, a clear priority among participating writers was to document both the frequency and the consequences of society's ongoing failures to notice, hear, include, and believe young women - as women, as youths, or as survivors.

This depiction of neglect and dismissal as forms of violence offers important context for findings related to young women's values and assets. For example, results indicated that most young women felt both an obligation and an ability to contribute to a safer and healthier community and society; the data further suggest that they saw this obligation and ability as necessarily shared by all—including institutions and legislators. ${ }^{95}$ In fact, across all data sets, hope emerged as a common and significant asset among young women, as they expressed belief that violence could be reduced, that beneficial actions could realistically be taken, and that it was possible to imagine a better,

\footnotetext{
${ }^{95}$ As opposed, for example, to being the concern strictly or primarily of victims, activists, youth, women, etcetera.
} 
safer world. Other assets emerging in the data included confidence, perseverance, resilience, and the desire (and ability) to encourage or support others.

At the same time, young women indicated that their potential power to contribute to a safer world was regularly nullified or controverted by: 1) institutional and societal failures to listen to young women, 2) institutional and societal dismissals of young women's concerns, 3) young women's lack of social supports, and 4) institutional and societal failures to hold institutions and perpetrators accountable-particularly in issues related to sexual assault, domestic and intimate partner violence, and gun violence. In other words, young women traced the perpetuation of violence in part to denials or dismissals of young women's knowledge and lived experience. This insight has significant implications for public health practice, which are further discussed in Chapter Five.

Young women's sense of being unheard or disbelieved was underlined by young women's primary values related to violence, which included: caring for others, justice, speaking and being heard, trust, and the need for meaningful, responsive action by society and institutions. As Richard Shaull wrote, "The young perceive that their right to say their own word has been stolen from them, and that few things are more important than the struggle to win it back" (Freire, 2005, p. 34).

In sum, the arts-based data addressed and expanded the forms of violence measured by the surveys, while providing insight into young women's own perceptions of barriers, assets, and potential solutions. ${ }^{96}$ The arts-based data placed young women's

\footnotetext{
${ }^{96}$ Proposed "solutions" to violence included: education (particularly about consent, gender roles, and relationships), policies addressing gun access, listening to and believing survivors, addressing addiction, confronting rape culture, and providing more safe spaces for youth.
} 
experiences of violence within broader contexts than the surveys allowed-particularly those of societal and institutional failures to hear, believe, protect, and include young women. ${ }^{97}$ Importantly, while providing clear indications of their experiences of violence, fear, and barriers to health, young women also provided clear indications of hope, strength, resilience, and potential for leadership and influence.

\subsection{Analysis of Prototypes: Initial Findings}

This section provides an initial assessment of the three methodological prototypes tested by this study: art resulting from a programmatic health intervention (YDS), art resulting from a citywide call for submissions (contest entries), and art created by and for a community, without researcher involvement (Wildfire). This assessment begins with a comparative analysis of findings regarding violence among young women ages 11 to 21 , including limitations and affordances of utilized methods. It then draws on the goals and theories explicated in Chapter One to assess the prototypes' abilities to meet ongoing methodological needs in public health inquiry. Finally, the three prototypes are assessed for the extent to which they offered the "untapped benefits" of arts-based research methods, as described in Chapter Two.

\subsubsection{Comparison of Data}

For a preliminary examination of the limitations and affordances of each method, findings from all methods were individually listed and cross-checked. For each method of data collection, each finding was given a rating of 0 (not addressed); 1 (potentially addressed, depending on how questions or data are interpreted); or 2 (clearly addressed in some way). The assignment of "2" to any given cross-section does not imply that the two

\footnotetext{
${ }^{97}$ Notably, this context was not addressed by the surveys except within the frameworks of "social supports" (pertaining to individual supports), and institutional responses to reports of sexual assault.
} 
methods addressed the issue similarly or equally well; instead, it offers a preliminary view of what which findings were able to emerge at all from each data source. To allow for quick visual inferences, these numbers have also been assigned colors; zero corresponds with red, one with yellow, and two with green. Full results are documented in Tables 4.27 and 4.28 .

Table 4.27: Methods Comparison I

\begin{tabular}{|l|c|c|c|c|}
\hline Arts-Based Findings & YDS & YVPRC & CardSafe & WBR \\
\hline $\begin{array}{l}\text { Experiences of } \\
\text { Violence: } \\
\text { Interpersonal }\end{array}$ & & & & \\
\hline $\begin{array}{l}\text { Domestic Violence, } \\
\text { IPV }\end{array}$ & 2 & 0 & 2 & 2 \\
\hline Trauma & 2 & 1 & 2 & 2 \\
\hline Trauma Symptoms & 2 & 1 & 0 & 2 \\
\hline Fear, Threat & 2 & 1 & 2 & 2 \\
\hline Loneliness, Isolation & 2 & 2 & 1 & 2 \\
\hline $\begin{array}{l}\text { Drugs, Substance } \\
\text { Abuse }\end{array}$ & 2 & 2 & 2 & 2 \\
\hline Guns, Gun Violence & 0 & 1 & 0 & 0 \\
\hline Grief, Loss & 1 & 0 & 0 & 1 \\
\hline Bullying, Labels & 1 & 1 & 2 & 1 \\
\hline $\begin{array}{l}\text { Sexual Assault, } \\
\text { Harassment }\end{array}$ & 2 & 0 & 2 & 2 \\
\hline Child Abuse & 2 & 0 & 0 & 1 \\
\hline Pain & 0 & 0 & 2 & 0 \\
\hline Inaction, Complacency & 0 & 2 & 2 & 1 \\
\hline Insecurity, Self-Doubt & 2 & 1 & 0 & 1 \\
\hline Betrayal & 0 & 0 & 1 & 0 \\
\hline Death & 0 & 0 & 0 & 1 \\
\hline Lack of Control & 0 & 1 & 2 & 0 \\
\hline $\begin{array}{l}\text { Experiences of } \\
\text { Violence: Structural }\end{array}$ & & & & \\
\hline Institutional Inaction & 0 & 0 & 2 & 0 \\
\hline
\end{tabular}




\begin{tabular}{|c|c|c|c|c|}
\hline School/Mass Shootings & 0 & 0 & 0 & 0 \\
\hline Incarceration & 1 & 0 & 0 & 0 \\
\hline $\begin{array}{l}\text { Homelessness, Hunger, } \\
\text { Poverty }\end{array}$ & 2 & 2 & 0 & 2 \\
\hline Sexism, Misogyny & 0 & 0 & 2 & 0 \\
\hline $\begin{array}{l}\text { Racism, White } \\
\text { Supremacy }\end{array}$ & 0 & 2 & 0 & 0 \\
\hline \multicolumn{5}{|l|}{ Barriers } \\
\hline Mental Health & 2 & 0 & 2 & 2 \\
\hline $\begin{array}{l}\text { Gender Roles, } \\
\text { Expectations }\end{array}$ & 0 & 0 & 0 & 0 \\
\hline No One to Depend On & 2 & 2 & 2 & 2 \\
\hline No One Notices & 0 & 1 & 1 & 1 \\
\hline No One Listens/Hears & 0 & 1 & 1 & 1 \\
\hline Deceptive Love & 0 & 0 & 1 & 0 \\
\hline \multicolumn{5}{|l|}{ Needs } \\
\hline $\begin{array}{l}\text { To ACT, take } \\
\text { responsibility }\end{array}$ & 0 & 2 & 2 & 1 \\
\hline Speaking Out, Voice & 0 & 0 & 2 & 1 \\
\hline Self-worth & 2 & 1 & 1 & 2 \\
\hline Social Supports & 1 & 2 & 2 & 2 \\
\hline Safety & 1 & 1 & 2 & 2 \\
\hline Self-care, Self-love & 1 & 0 & 0 & 2 \\
\hline Access to Resources & 0 & 0 & 2 & 2 \\
\hline To Express, Share & 1 & 0 & 0 & 0 \\
\hline Education & 0 & 1 & 1 & 2 \\
\hline \multicolumn{5}{|l|}{ Effects } \\
\hline $\begin{array}{l}\text { Guilt, Shame, Regret, } \\
\text { Self-Blame }\end{array}$ & 0 & 0 & 1 & 0 \\
\hline $\begin{array}{l}\text { Hopelessness, } \\
\text { Overwhelm }\end{array}$ & 2 & 1 & 1 & 2 \\
\hline Lingering Effects & 2 & 0 & 2 & 2 \\
\hline Bruises, Scars & 0 & 0 & 1 & 0 \\
\hline Anger, Rage & 2 & 1 & 0 & 0 \\
\hline
\end{tabular}




\begin{tabular}{|l|c|c|c|c|}
\cline { 2 - 5 } Pretending, Hiding & 1 & 0 & 1 & 0 \\
\hline Tears & 0 & 0 & 0 & 0 \\
\hline Memories & 1 & 0 & 0 & 0 \\
\hline Sensory Details & 0 & 0 & 1 & 0 \\
\hline Uncertainty & 0 & 0 & 1 & 2 \\
\hline Numb, Numbness & 2 & 0 & 1 & 0 \\
\hline Scream, Can't Scream & 0 & 0 & 0 & 0 \\
\hline $\begin{array}{l}\text { "Had to grow up too } \\
\text { fast" }\end{array}$ & 0 & 0 & 0 & 0 \\
\hline "The BODY speaks" & 1 & 0 & 0 & 1 \\
\hline Attributes & & 1 & & \\
\hline Authenticity, Inner self & 0 & 0 & 0 & 2 \\
\hline Claiming or Defining & 2 & 2 & 0 & 2 \\
\hline Innocence & 0 & 0 & 0 & 0 \\
\hline $\begin{array}{l}\text { Vulnerability, } \\
\text { weakness }\end{array}$ & 0 & 1 & 1 & 1 \\
\hline Beauty, beautiful & 0 & 0 & 0 & 0 \\
\hline Smart, not smart & 0 & 1 & 0 & 1 \\
\hline $\begin{array}{l}\text { Assets } \\
\text { Confidence, } \\
\text { Empowerment }\end{array}$ & 1 & 2 & 0 & 2 \\
\hline Hope & 2 & 2 & 0 & 2 \\
\hline $\begin{array}{l}\text { Encouraging/Advising/ } \\
\text { Advocating for others }\end{array}$ & 2 & 1 & 0 & 1 \\
\hline Resilience & 2 & 2 & 1 & 2 \\
\hline $\begin{array}{l}\text { Perseverance, } \\
\text { Persistence }\end{array}$ & 2 & 1 & 0 & 2 \\
\hline $\begin{array}{l}\text { Imagination/Dreams } \\
\text { (for the future) }\end{array}$ & 0 & 1 & 0 & 1 \\
\hline $\begin{array}{l}\text { Healing, } \\
\text { Transformation }\end{array}$ & 0 & 0 & 0 & 1 \\
\hline Support from others & 1 & 2 & 1 & 2 \\
\hline Strength & 1 & 1 & 0 & 1 \\
\hline Courage, Leadership & 0 & 1 & 0 & 1 \\
\hline
\end{tabular}




\begin{tabular}{|l|c|c|c|c|}
\cline { 2 - 5 } $\begin{array}{l}\text { Learning from } \\
\text { trauma/pain }\end{array}$ & 0 & 0 & 0 & 1 \\
\hline Forgiveness & 0 & 1 & 0 & 0 \\
\hline Values & & & & \\
\hline Caring for others & 2 & 1 & 1 & 2 \\
\hline Justice, injustice & 0 & 2 & 1 & 0 \\
\hline Trust, Loyalty & 0 & 1 & 0 & 1 \\
\hline Faith / God & 0 & 0 & 0 & 0 \\
\hline Kindness & 1 & 1 & 0 & 1 \\
\hline Respect & 0 & 1 & 1 & 2 \\
\hline Beliefs & 1 & 1 & 0 & 1 \\
\hline Light vs Darkness & 0 & 0 & 0 & 0 \\
\hline Love & 0 & 0 & 1 & 1 \\
\hline Standing up & 0 & 2 & 2 & 1 \\
\hline "shouldn't have to" & 0 & 1 & 0 & 0 \\
\hline Diversity & 0 & 1 & 1 & 1 \\
\hline TOTAL & $\mathbf{6 1}$ & $\mathbf{5 9}$ & $\mathbf{6 2}$ & $\mathbf{8 1}$ \\
\hline Percentage & $\mathbf{3 7 \%}$ & $\mathbf{3 6 \%}$ & $\mathbf{3 8 \%}$ & $\mathbf{4 9 \%}$ \\
\hline
\end{tabular}

Table 4.28: Methods Comparison II

\begin{tabular}{|l|c|c|c|}
\hline \multicolumn{1}{|c|}{ Quantitative Findings } & YDS & Contest & Wildfire \\
\hline YDS Survey & & & \\
\hline Demographics & 2 & 2 & 1 \\
\hline Divorce/Separation & 1 & 2 & 2 \\
\hline $\begin{array}{l}\text { Incarceration of family } \\
\text { member }\end{array}$ & 1 & 2 & 1 \\
\hline Verbal abuse & 2 & 2 & 2 \\
\hline Domestic Violence & 1 & 2 & 2 \\
\hline Sense of neglect & 2 & 2 & 2 \\
\hline Drugs In home & 1 & 1 & 1 \\
\hline Mental illness in home & 0 & 2 & 1 \\
\hline Trauma symptoms & 2 & 2 & 2 \\
\hline PTSD & 2 & 2 & 2 \\
\hline
\end{tabular}




\begin{tabular}{|l|c|c|c|}
\cline { 2 - 4 } Resilience & 2 & 2 & 2 \\
\hline TOTAL & 16 & 21 & 18 \\
\hline YVPRC Survey & & & \\
\hline Demographics & 2 & 2 & 1 \\
\hline Community violence & 1 & 2 & 0 \\
\hline Witnessing violence & 1 & 2 & 2 \\
\hline $\begin{array}{l}\text { Responsibility to help } \\
\text { community }\end{array}$ & 1 & 2 & 2 \\
\hline Empowerment, Efficacy & 2 & 2 & 2 \\
\hline $\begin{array}{l}\text { Hope (re: violence } \\
\text { reduction) }\end{array}$ & 1 & 2 & 2 \\
\hline Personal action & 2 & 2 & 2 \\
\hline Attitudes about violence & 2 & 2 & 2 \\
\hline Social supports & 2 & 2 & 2 \\
\hline $\begin{array}{l}\text { Correlations: Age or } \\
\text { Grade level }\end{array}$ & 0 & 2 & 0 \\
\hline Correlations: Race & 2 & 2 & 0 \\
\hline $\begin{array}{l}\text { Correlations: Variable to } \\
\text { variable }\end{array}$ & 1 & 1 & 0 \\
\hline TOTAL & $\mathbf{1 7}$ & $\mathbf{2 3}$ & $\mathbf{1 5}$ \\
\hline $\begin{array}{l}\text { CardSafe Survey } \\
\text { (Limited Demographics) }\end{array}$ & 2 & 2 & 1 \\
\hline Sexual harassment & 1 & 2 & 2 \\
\hline Bullying & 2 & 2 & 1 \\
\hline Verbal abuse & 0 & 2 & 2 \\
\hline Physical abuse & 0 & 0 & 1 \\
\hline Stalking & 1 & 2 & 2 \\
\hline $\begin{array}{l}\text { Monitoring, Invading } \\
\text { privacy }\end{array}$ & 0 & 1 \\
\hline Being threatened & 2 & 2 \\
\hline Unwanted sex & & 2 \\
\hline $\begin{array}{l}\text { Intimate Partner Violence } \\
\text { (IPV) }\end{array}$ & 0 & 2 \\
\hline Being drugged & & \\
\hline
\end{tabular}




\begin{tabular}{|c|c|c|c|}
\hline $\begin{array}{l}\text { Hearing someone brag } \\
\text { about rape or sexual } \\
\text { assault }\end{array}$ & 0 & 0 & 0 \\
\hline $\begin{array}{l}\text { Education about reducing } \\
\text { violence }\end{array}$ & 1 & 2 & 2 \\
\hline TOTAL & 9 & 20 & 20 \\
\hline \multicolumn{4}{|l|}{ WBR Survey } \\
\hline Demographics & 2 & 2 & 1 \\
\hline Safety (vs Fear/Threat) & 2 & 2 & 2 \\
\hline Suicide ideation & 1 & 2 & 2 \\
\hline Sexual/relational violence & 1 & 2 & 2 \\
\hline Hopelessness & 2 & 2 & 2 \\
\hline $\begin{array}{l}\text { Difficulty making it } \\
\text { through stressful events }\end{array}$ & 1 & 2 & 2 \\
\hline Bodily appearance & 2 & 2 & 2 \\
\hline Perceived personal health & 0 & 1 & 2 \\
\hline Correlations: Race & 1 & 2 & 0 \\
\hline $\begin{array}{l}\text { Correlations: Sexual } \\
\text { orientation }\end{array}$ & 1 & 0 & 0 \\
\hline Access to healthcare & 0 & 2 & 2 \\
\hline Valued identity & 2 & 2 & 2 \\
\hline $\begin{array}{l}\text { Long-term effects (inc. } \\
\text { academic success) }\end{array}$ & 1 & 2 & 2 \\
\hline $\begin{array}{l}\text { Sense of } \\
\text { purpose/meaning }\end{array}$ & 2 & 2 & 2 \\
\hline $\begin{array}{l}\text { Contribute to others' } \\
\text { well-being }\end{array}$ & 2 & 2 & 2 \\
\hline Good person, good life & 2 & 1 & 1 \\
\hline Competence, capability & 2 & 2 & 2 \\
\hline $\begin{array}{l}\text { Optimism about the } \\
\text { future }\end{array}$ & 2 & 2 & 2 \\
\hline Overcoming difficulty & 2 & 2 & 2 \\
\hline TOTAL & 28 & 34 & 32 \\
\hline Overall Totals & 70 & 98 & 85 \\
\hline & $64 \%$ & $89 \%$ & $77 \%$ \\
\hline
\end{tabular}


As noted in Table 4.27, the YDS survey was able to address 37 percent of the findings from the arts-based data, while the YVPRC survey addressed 36 percent, and the CardSafe survey addressed 38 percent. The Well-Being and Resilience survey had the most comparable findings, addressing 49 percent of the codes emerging from the artsbased data.

As noted in Table 4.28, the YDS writing was able to address 64 percent of the findings from the survey data, while writing contest data addressed 89 percent, and the Wildfire play addressed 77 percent. The relatively low rate of alignment between YDS writing and the surveys can be attributed to the fact that the intervention from which this population's writing was drawn was not violence-focused. Unlike writers in the other samples, the writers in detention were given many ideas and prompts about which to write. As a result, the resulting data set was, on the whole, less likely to offer details or specifics regarding violence.

\subsubsection{Survey Methods: Initial Results}

The surveys were most comparable to creative writing in their abilities to indicate respondents' experiences of interpersonal violence, potential needs, and assets such as hope or resilience. These affordances were primarily available from the YDS and WBR surveys, which intentionally focused on trauma and resilience; the other surveys did not fare as well.

A clear affordance of the survey method was the ability to examine correlations among variables. As noted above, similar tests may be possible with certain kinds of creative data sets; for example, given that contest entrants provided demographic information when submitting writing entries, resulting codes could be disaggregated by 
demographic variables to allow for an examination of disparities. However, as noted in Chapter Two, the issues illuminated by the field's increasing focus on social determinants and the social ecological model are more akin to social than natural sciences, requiring methods "involved in comprehending difficult or obscure communications and not of the sort involved in attaining valid statistical generalisations [sic]" (Hughes and Sharrock, 1997, p. 20). In other words, when researching socially-constructed, health-determinative concerns such as violence, the ability to run statistical tests need not continue to be public health's primary or preferred means of knowledge production or validation. However, given that researchers are unlikely to be forced to choose between these "sorts" of inquiry, the goal of innovation is to enlarge and critically examine-not replace-current methodological options and assumptions.

Another affordance of the surveys was the ability to obtain responses to direct, specific questions - such as whether an individual had heard another student brag about rape. Such specific occurrences were unlikely to arise in creative writing without (more) direct prompting. This affordance is the mirror image of surveys' primary limitationwhich is that they elicit only those specific details that researchers or administrators had predetermined as valuable. They are thus more adept at revealing the extent, prevalence, and correlating factors regarding a pre-defined issue than at revealing those issues, experiences, or concerns that exist for the target population. ${ }^{98}$ In addition, although surveys can provide answers to specific questions, limited response options may at times render those answers inaccurate. As noted in the example above about intervening when a

\footnotetext{
${ }^{98}$ This is a broadly-understood limitation of survey data, which is why surveys are often constructed following interviews, focus groups, listening sessions, or other means of qualitative data collection. As this study illuminates, the arts appear to offer similar qualitative data.
} 
fellow student is being hurt, individual answers to the question were highly dependent upon respondents' varied interpretations of its meaning.

The final affordance of the survey method in the current study was the greater availability of demographic data, based in part on anonymity. As mentioned earlier, the writing contest did elicit demographic data from entrants, and future projects or studies could elicit similar information for integration into analyses. However, the survey method currently has the advantage of a social norm, in that survey participants are likely accustomed to being asked for demographic information when completing questionnaires, while writers and artists are not accustomed to providing such information in the context of creating. In addition, art involves intellectual property concerns that are not relevant to most surveys. Creators may often wish to attach their name to a work of art; however, this attachment of a name to a work may render the artist reticent to offer additional information — since that information would not be anonymous. Given that trauma- and cultural-responsiveness entails awareness of individuals' comfort levels in sharing not only their creative work but also details about their person, future public health work with creative-based data will need to examine whether-and how best— to collect additional information.

\subsubsection{New Methods: Initial Results}

The creative data were most comparable to survey data in their ability to identify experiences of interpersonal violence (personal or witnessed), as well as attitudes and beliefs related to themselves, their communities, and safety. These affordances were primarily available from the contest and Wildfire writings, which had intentionally 
focused on violence and healing; the YDS writings were broader and thus less consistently correspondent with survey findings.

A distinct advantage of the arts-based data was their ability to identify and even foreground unexpected or less typical experiences of violence or harm, such as trauma symptoms, gun violence, grief, bullying, sexual assault, child abuse, pain, inaction, death, betrayal, and lack of control. While surveys cannot be expected to predict or address every possible form of violence, the clear benefit of creative writing in this study was the ability for such experiences to emerge on their own - without an overly-extensive survey, and without having to have been previously known by researchers.

Another distinct advantage was the creative data's ability to illuminate structural barriers, such as in writers' mentions of institutional inaction, sexism, and incarceration. By contrast, the surveys were largely focused on individual experiences-with structural causes or barriers left to be deduced by researchers. An additional affordance of the creative data was its illumination of attributes. Not only were writers able to claim their own identities and descriptors, which increased the methods' trauma- and cultural responsiveness, but writers' identification and descriptions of their attributes adds to researcher understandings of young women's priorities and felt experiences. This improves future development of responses and interventions. For example, the prominence of authenticity/inner self in the creative data indicated a common sense of disconnection from oneself, or of an inability to be heard and seen $a$ s that self. Opportunities for reconnection may thus have more implications for population health and safety than previously recognized. 


\subsubsection{Assessing the New Methods}

According to Design Thinking, the goal of prototyping new methods is to determine quickly the potential value of a new approach to an identified need; in this case, that need was for methods of public health inquiry and data collection that are more trauma- and culturally-responsive. Initial results indicate that the creative data in this study delivered insightful, qualitative data comparable to those obtained in other studies using conventional methods such as focus groups or interviews. However, the tested methods accessed these data more quickly and cost-effectively than most qualitative research processes—and involved minimal researcher intrusion. Below, these methods are assessed for their accommodation of theory-grounded needs and considerations noted in earlier chapters.

\subsubsection{Desired Outcomes}

Chapter One indicated that new public health research methods were needed, which would expand expressive options, increase trauma- and cultural responsiveness, and increase accessibility and inclusion. Findings regarding these outcomes, for each of the three methods of data collection, are documented in Table 4.29.

Table 4.29: Assessment of Desired Outcomes

\begin{tabular}{|l|l|l|l|}
\hline Goal & \multicolumn{1}{|c|}{ YDS Writing } & \multicolumn{1}{c|}{ Contest } & \multicolumn{1}{c|}{ Wildfire } \\
\hline $\begin{array}{l}\text { Expanded } \\
\text { expressive } \\
\text { options? }\end{array}$ & $\begin{array}{l}\text { Poetry, song lyrics, } \\
\text { letters }\end{array}$ & $\begin{array}{l}\text { Poetry and short } \\
\text { essays }\end{array}$ & $\begin{array}{l}\text { Play script, } \\
\text { performance }\end{array}$ \\
\hline $\begin{array}{l}\text { Trauma- } \\
\text { responsive? }\end{array}$ & $\begin{array}{l}\text { Writing activities } \\
\text { were completed as } \\
\text { part of a trauma- } \\
\text { informed program. }\end{array}$ & $\begin{array}{l}\text { Writing and } \\
\text { entering the contest } \\
\text { was optional, so } \\
\text { individuals } \\
\text { participated only if } \\
\text { they wished. } \\
\text { Similizing the } \\
\text { resulting data } \\
\text { allowed the }\end{array}$ & $\begin{array}{l}\text { Utilizing these data } \\
\text { allows researchers to } \\
\text { learn about the } \\
\text { writers' experiences of } \\
\text { trauma on their terms, } \\
\text { without intruding } \\
\text { upon them in any way, }\end{array}$ \\
\hline
\end{tabular}




\begin{tabular}{|c|c|c|c|}
\hline & $\begin{array}{l}\text { researcher to identify } \\
\text { new concerns among } \\
\text { the population } \\
\text { without intruding } \\
\text { further upon a } \\
\text { vulnerable } \\
\text { population, such as } \\
\text { with more surveys, } \\
\text { or by conducting } \\
\text { interviews. }\end{array}$ & $\begin{array}{l}\text { prompt was kept } \\
\text { broad for } \\
\text { maximum content } \\
\text { choice. }\end{array}$ & $\begin{array}{l}\text { and without having to } \\
\text { ask them to share their } \\
\text { stories again. }\end{array}$ \\
\hline $\begin{array}{l}\text { Culturally } \\
\text { responsive? }\end{array}$ & $\begin{array}{l}\text { Participants were } \\
\text { regularly exposed to } \\
\text { diverse artists, } \\
\text { authors, musicians; } \\
\text { prompts encouraged } \\
\text { writers to express } \\
\text { themselves in } \\
\text { whatever ways felt } \\
\text { more natural or } \\
\text { rewarding. }\end{array}$ & $\begin{array}{l}\text { Prompts were kept } \\
\text { broad for } \\
\text { maximum } \\
\text { response/choice. } \\
\text { Submissions } \\
\text { illustrated great } \\
\text { diversity in style, } \\
\text { length, approach, } \\
\text { literacy levels, } \\
\text { storytelling } \\
\text { techniques, etc. }\end{array}$ & $\begin{array}{l}\text { Utilizing these data } \\
\text { was a means of } \\
\text { attending to current } \\
\text { cultural phenomena in } \\
\text { the community, } \\
\text { including original } \\
\text { work by female artists } \\
\text { responding (and } \\
\text { contributing) to } \\
\text { \#MeToo. }\end{array}$ \\
\hline Accessible? & $\begin{array}{l}\text { Intervention was } \\
\text { attentive to varying } \\
\text { literacy levels, as } \\
\text { well as means by } \\
\text { which young women } \\
\text { may wish to express } \\
\text { themselves (writing } \\
\text { or otherwise), given } \\
\text { the difficult context } \\
\text { of detention. }\end{array}$ & $\begin{array}{l}\text { The contest } \\
\text { required access to a } \\
\text { computer or } \\
\text { internet, and ability } \\
\text { to type one's work } \\
\text { (or have it typed). } \\
\text { Contest hosts } \\
\text { allowed } \\
\text { handwritten entries } \\
\text { to be scanned and } \\
\text { emailed by } \\
\text { teachers whose } \\
\text { students lacked } \\
\text { access. }\end{array}$ & $\begin{array}{l}\text { N/A; data were } \\
\text { secondary. }\end{array}$ \\
\hline Inclusive? & $\begin{array}{l}\text { All female- } \\
\text { identifying residents } \\
\text { at the detention } \\
\text { center are welcomed } \\
\text { to write as part of } \\
\text { this intervention. } \\
\text { Intervention includes } \\
\text { regular inclusion of } \\
\text { works by girls' } \\
\text { preferred writers, } \\
\text { musicians, actors, or }\end{array}$ & $\begin{array}{l}\text { Explicitly open to } \\
\text { all female- } \\
\text { identifying } \\
\text { individuals ages } 11 \\
\text { to } 21 \text {, living in the } \\
\text { Metro Louis ville } \\
\text { area. While widely } \\
\text { and heavily } \\
\text { promoted, it is } \\
\text { possible that girls } \\
\text { out of school or }\end{array}$ & $\begin{array}{l}\text { N/A; data were } \\
\text { secondary. }\end{array}$ \\
\hline
\end{tabular}




\begin{tabular}{|l|l|l|}
$\begin{array}{l}\text { other artists; these } \\
\text { are referenced as } \\
\text { writing inspirations } \\
\text { or topics of } \\
\text { discussion. }\end{array}$ & $\begin{array}{l}\text { unaffiliated with } \\
\text { city organizations } \\
\text { may have missed } \\
\text { the call for } \\
\text { submissions. }\end{array}$ & \\
\hline
\end{tabular}

\subsubsection{Potential Benefits of Arts-Based Methods}

Chapter Two indicated that arts-based methods have the ability to: 1) aid disclosure (thereby improving data), 2) offer direct benefits (thereby improving both participant health and research reciprocity), and 3) create more opportunities for public education and awareness. Regarding aided disclosure, writers in all three data sets appear to have shared ideas or experiences that may have been difficult to share otherwiseeither because of the content's personal or emotional nature, or because it would not have been prompted by researchers. This is particularly true for the Wildfire writers, who disclosed very personal, often graphic details regarding their experiences of sexual assault. Their ability to do so appears to have been linked to the performance aspect of their creative medium, which turned disclosures into opportunities to help or encourage other survivors while educating the broader public.

Regarding direct health benefits, it is likely_-based on the literature regarding expressive writing ${ }^{99}$ - that young women in all three populations received some health benefit from processing their feelings or experiences via writing. Opportunities for public education and awareness were also clearly made available by all three methods. For example, portions of writing from YDS were published in a book by Sarabande Books and disseminated throughout the community; they will also be mailed to local/state

\footnotetext{
${ }^{99}$ See Chapter One, section 1.6.2, and Chapter Two, section 2.6.1.
} 
legislators, employing girls' own voices to help inform youth and justice policies/resources. Similarly, portions of writing from the National Youth Violence Prevention Week writing contest were published in a contest anthology by Sarabande Books, which was disseminated throughout the community. Contest winners also read their poetry live on local radio and at a public book-launch event; these opportunities together elevated young women's particular experiences and perceptions of violence. Finally, How to Start a Wildfire was performed at a University of Louisville theater on two nights, which were both sold out. This created a unique opportunity for communityled education and awareness-raising about sexual assault.

\subsection{Conclusion}

This chapter detailed findings about violence from three sets of arts-based data, which included eight themes that were grouped according to four categories informed by the research questions. It then presented findings from four related surveys, offering comparisons throughout to previously presented findings from the creative data. The latter part of the chapter compiled and cross-checked findings across data sets, providing an initial assessment of methodological affordances and limitations; it then offered an initial assessment regarding the extent to which the tested methods met needs and considerations identified in Chapters One and Two. A discussion of these findings, including implications and recommendations for further research, is offered in Chapter Five. 


\section{CHAPTER FIVE: DISCUSSION}

This dissertation began with an overview of the field's need for innovation to improve cultural- and trauma-responsiveness - not only in interventions and health promotion campaigns, but in research methodologies and practices themselves. Chapter Two reviewed the biomedical and social ecological models, arguing that the assumptions of the former (positivism, dualism, reductionism) are unlikely to support the needs of the latter, which include the abilities to: increase inclusivity and access; elicit and apply diverse knowledges; ascertain the health-determinative effects of social and other contextual factors; and identify the extent to which health realities-including health disparities - are affected or determined by disparate attributions of meaning and value. ${ }^{100}$ As a result, there exists an urgent need for new methods designed to "understand others, especially those from whom we are historically [or] culturally remote" (Hughes \& Sharrock, 1997).

As a model for such methods, Chapter Two presented a review of photovoice, noting how its benefits and drawbacks illuminate the potential for more trauma- and

\footnotetext{
${ }^{100}$ As public health priorities have shifted from individual-focused efforts to the analysis and promotion of health determinants such as housing, education, transportation, or "health in all policies," it is increasingly clear that health and well-being cannot be adequately understood or addressed apart from historical, social, and cultural contexts (including "root causes" of health disparities); lived experiences; individual and population-level meaning-making strategies; and, more generally, researchers' ongoing reflexivity regarding methods of public health inquiry. For example, the ways in which populations' histories, perceptions, experiences, and meaning-making practices influence or determine their health and health behaviors may not be noticed or adequately understood by traditional research practices.
} 
culturally-responsive research practices that increase inclusivity, access, data quality, and research reciprocity. Building on these considerations, the researcher created and tested three methodological prototypes for researching the needs, assets, priorities, and values of a vulnerable population (young women), related to a potentially traumatizing topic (violence). The prototypes differed importantly in method of data elicitation or collection. The first involved collecting writing as part of an ongoing, arts-based health intervention in a local youth detention facility. The second involved partnering with a local community organization for National Youth Violence Prevention Week, to create a citywide creative writing contest that widely promoted participation from young women ages 11 to 21 throughout the Louisville Metro area. The third involved identifying and obtaining access to local art related to the research topic. This was the script for a play about sexual assault that been written and performed at a local university without researcher intervention or elicitation.

These prototypes were developed utilizing the Public Health Innovation Model, created by Lister et al. (2017) — who argued that "[i]nnovation is not intended to replace public health best-practices or planning models but is available to enhance those practices and tailor interventions to meet local needs" (p. 7). Therefore, the purpose of this nested, mixed methods study was not to replace existing or preferred methods, but rather to clarify the need for ongoing methodological innovation, to model said innovation, and to examine the value of three prototyped methods in enhancing data quality, traumaresponsiveness, and cultural-responsiveness. To that end, this study examined: 1) the extent to which these new methods could inform researchers about populations' experiences, needs, assets, and priorities related to a potentially traumatizing issue; and 2) 
how these methods compared to traditional survey methods - in quality and quantity of data as well as in trauma- and cultural-responsiveness.

This final chapter includes a summary and interpretation of major findings, placing them in the context of literature referenced in earlier chapters regarding traumaresponsiveness, cultural-responsiveness, intersectional theory, epistemological assumptions, and the potential benefits of including creative arts. This chapter additionally discusses implications of these findings for the field, before covering study limitations and recommendations for future research.

\subsection{Summary of Findings}

Looking at the three prototypes as a combined example of arts-based data collection, their findings contributed more nuanced health information than the related surveys; they were additionally able to address 64 to 89 percent of the same variables as the surveys. (By contrast, the surveys addressed only 36 to 49 percent of the variables addressed in the arts-based data sets.) ${ }^{101}$ Like qualitative methods more generally, the prototyped methods were particularly adept at: identifying experiences and issues that may not previously have been considered by researchers or "outsiders;" revealing participant identities and perceptions; and placing identified needs in the context of participants' assets and attributes. However, in contrast to qualitative methods such as interviews or focus groups, these arts-based practices involved minimal researcher intrusion, were time- and cost-effective, and offered greater potential for reciprocity-

\footnotetext{
${ }^{101}$ Of course, each survey had its own originating goals which did not necessarily align with that of this project, which was to understand young women's experiences and perceptions of violence. However, they do exemplify the types of questions asked and answered in traditional surveys, and what researchers are able to glean (or not) from such answers.
} 
such as through direct health benefits, support for creative and cultural expression, and the local publication and elevation of participants' original work. The new methods also further expanded expressive options, inviting creativity, metaphor, abstraction, and a range of approaches (from relating personal experiences to sharing broad perceptions of society). These affordances appear to have together aided participant disclosure of difficult experiences or concerns - thus expanding what could be learned about the sample populations.

Separating out the three prototypes, contest writing delivered relevant, extensive data from across the target metropolitan area; these data were grounded in personal expressive choice, with no researcher involvement until after participants' writing and contest-submission processes had already been completed. This indicates the value of creative events - along with broad calls for participation — in generating significant qualitative data while remaining trauma- and culturally-responsive. Participants in the ongoing intervention at Youth Detention Services (YDS) were also offered extensive personal expressive choice, indicating trauma- and cultural-responsiveness; in addition, the data provided by their writing indicate that creative arts interventions can be designed to function not only for health provision or education, but to simultaneously generate data useful beyond the intervention itself. Finally, analysis of the play How to Start a Wildfire indicated that a target population's recent, pre-existing art is capable of addressing current research questions. In fact, to the extent that an artwork's themes align with research questions, pre-existing creative content can provide very personal and insightful data about difficult or stigmatized experiences, without any researcher intrusion at all. Each of 
the prototyped methods provided unique opportunities for public education — including peer education — which can be utilized to increase local issue and resource awareness.

\subsection{Interpretation and Contextualization of Findings}

\subsubsection{Comparing Surveys to Prototyped Methods}

This study found the surveys to be superior to the prototyped methods in accommodating measurements of the extent, prevalence, and correlating factors related to pre-defined issues related to violence. They were also superior in the provision both of demographic data and of answers to very specific questions. These findings reflect the value placed on these methods in epidemiological research, particularly regarding the determination of causal factors.

However, the surveys were inferior to the prototyped methods in revealing what issues, experiences, or concerns actually existed among — and were prioritized by — the target population. To the extent that local qualitative data were used to determine each survey's instruments, items, and overarching design, (in other words, to the extent that the predefined issues inquired about on the surveys were generated by the target populations themselves), this particular lack does not present a risk to equitable and accurate representations of population experiences and concerns. It instead affirms "the limited, partial, and situated" nature of research practices and findings (Shotter, 2014, p. 321), and the concomitant necessity of utilizing multiple methods for optimal—and optimally equitable—research design. ${ }^{102}$

\footnotetext{
${ }^{102}$ As noted in Chapter One, "[t]o be convinced that one perspective delivers the truth is to be blinded to the truths offered by others" (Feyeraband, 2011, p. x).
} 


\subsubsection{The Importance of Localized, Qualitative Data}

However, this study did reveal that greater localized data was indeed needed to increase the equity and accuracy of at least two of the surveys. For example, domestic and intimate partner violence was the priority experience in writing contest data, yet items related to this form of violence were wholly absent from the district-wide survey administered by the YVPRC. As a result, rates of exposure to violence indicated by survey results are almost certainly too low, and resulting assessments of potential health impacts experienced by the city's youth will inevitably be inaccurate. Should local resource decisions be informed by current survey results, they may fail to provide responses to forms of violence disproportionately experienced by women. ${ }^{103}$

Similarly, the survivors of sexual assault who wrote the play How to Start a Wildfire placed significant priority on communicating their experiences within the context of assets, goals, and positive social action. This is in keeping with literature on trauma-informed approaches, which suggests that contexts of strength, resilience, and empowerment may be needed for survivors to feel control and safety with regard to their disclosures. ${ }^{104}$ By contrast, the CardSafe survey sought extensive data regarding survivors' experiences of violence, but included no opportunities for survivors to communicate strengths, goals, or healing processes. As a result, the survey's design was

\footnotetext{
${ }^{103}$ Napier et al. (2014) asked, "How can [health] carers know what they are missing if they position themselves only to listen to what they are prepared to hear? ... [T] he empty survey form that comes back to school in the backpack of the same vulnerable child it went home with cannot inform; but the study that it is a part of can mislead" (p. 1626).

${ }^{104}$ SAMHSA (2014) notes that being trauma-informed requires using strengths-based approaches; Baker et al (2016) note that it requires "promot[ing] empowerment and resilience" (p. 13).

It is worth noting that, when given complete autonomy over their disclosures, and no researcher-intrusion, survivors naturally told their stories in trauma-informed ways. This supports the participatory action research value of recognizing participants as the experts in their own lived experiences (Curtis, Bryce, \& Treloar, 1999, p. 203), and that they often know better than outsiders what is needed for their safety and well-being.
} 
not adequately trauma-informed; it may also have precluded greater participation and more accurate results. ${ }^{105}$

In both of these cases, it is clear that greater access to and incorporation of relevant, local, qualitative data would have improved the surveys' designs, results, and impacts. Unfortunately, conventional qualitative research tends to be time- and costintensive - a fact which supports the argument in this dissertation for innovating with regard to how qualitative data is collected. In contrast to interviews and focus groups, the prototyped methods were relatively quick and cost-effective; they also likely appealed to different participants than conventional formats. They also came with "built-in" benefits such as peer education and awareness-raising through performances and publications.

\subsubsection{Moving Health Beyond the Individual}

As Knight (2014) argued, "the field...needs to gather and share data to tell a different kind of story that shifts the focus from individuals to communities and from behavior to structures" (p. 193). Because the analyzed surveys were individual-focused, they did not accommodate the emergence of participant knowledge related to structural and societal factors. ${ }^{106}$ By contrast, the arts-based data frequently included references to sexism, racism, incarceration, political inaction, childhood trauma, and other upstream causes of violence, as young women placed their violence-related experiences and thoughts in social ecological contexts. The writers' desire and ability to provide such contexts suggests that this knowledge can and should be sought; indeed, the ability for marginalized populations to recognize, name, and transform their broader situation may

\footnotetext{
105 The survey did provide an introduction with content warnings and reminders that participants could opt out; however, its content was deficits-focused, and did not include items related to resilience, strength, or empowerment.

${ }^{106}$ The YVPRC survey's inclusion of items related to "Thoughts on Society" is a notable exception.
} 
be fundamental to equitable and sustainable change. ${ }^{107}$ In other words, health equity and adequate social ecological responses may require not only that public health expand participative and communicative options, but also that it broaden the types of knowledge it typically seeks, to include subjects' own perceptions of causal factors.

\subsubsection{Expanding Terms and Inclusivity}

In addition to providing nuance and context precluded by survey-based methods, the prototyped methods also identified unexpected and atypical experiences of violence or harm, including insecurity, failures to be heard or believed, trauma, loneliness, and inaction, among others. As a result, the term "violence" was expanded to better recognize sources of harm originating in structural issues such as sexism or institutional paralysis. This helped identify a shift in the issue of prevention from individual to structural frameworks.

While expanding the definition of violence risks muddying communication related to the topic, it is more likely to improve accuracy by including active causes of destruction or harm. In addition, drawing upon earlier arguments regarding the need for greater inclusivity in knowledge-making practices, the fact that such harms have not regularly been recognized or discussed as forms of violence - the fact that "violence" has heretofore been more narrowly conceived - likely has more to do with failures to hear, believe, and act upon experiences of harm that differ from dominant expectations than with the scientific community's commitment to conceptual accuracy. In other words, the additional forms of violence illuminated by the current study provide an example of the

\footnotetext{
${ }^{107}$ According to Paulo Freire, "To surmount the situation of oppression, people must first critically recognize its causes, so that through transforming action they can create a new situation, one which makes possible the pursuit of a fuller humanity" (Freire, 2005, p. 47).
} 
need to challenge researchers' assumptions, and the need for additional means of hearing from marginalized populations. By providing such means, the prototyped methods supported the arguments in Chapters One and Two for methods specifically designed to understand "those from whom we are historically [or] culturally remote" (Hughes \& Sharrock, 1997), and to elicit and reflect "different kinds of knowledge inherent in communities" (Knight, 2014, p. 193).

\subsubsection{Unique Benefits}

\subsubsection{Trauma- and Cultural-Responsiveness}

As noted in Chapter Four, ${ }^{108}$ the prototyped methods expanded expressive options, thus aiding disclosure, and likely generated health benefits associated with expressive writing, self-efficacy, and performance. In addition, the elicitation of creative responses to particular prompts or themes required minimal outsider intrusion among the target populations, ${ }^{109}$ accommodated relative communicative choice, and recognized and valued the populations' creative and cultural productions. Although the creative writing data sets revealed extensive needs and barriers among participants, often with disturbing detail, they did so on participants' own terms, and frequently within the context of participants' own claimed identities and assets. Together, these factors suggest traumainformed, culturally-responsive practice.

\subsubsection{Increased Interest, Participation}

In addition, the use of creative formats (writing program, creative writing contest, play/performance) appears to have increased interest, access, and participation. For

\footnotetext{
108 See Chapter Four, Section 4.9.4.2.

${ }^{109}$ While the YDS data resulted from an intervention - and thus a form of researcher-intrusion - the data analyzed in the current study was already a fundamental aspect the intervention, and therefore required no additional intrusion.
} 
example, 105 individuals submitted poems about violence to the writing contest; it is not clear that a similar number of youth would have been interested in contributing to simple open-ended online survey questions about violence, without a tie-in to creativity, a contest, or potential publication. In other words, the interest garnered by the creative process, along with its potential for reciprocity, appear to have generated unique interest and participation. As argued earlier in the dissertation, health equity demands that researchers consider the extent to which the very nature of inquiry and data collectionincluding surveys, focus groups, or interviews - may preclude study access or participation, or stifle disclosures of painful or stigmatized experiences and their effects. Given that the prototypes created new forms and formats for participation and disclosure, they represent the value of innovation and creativity in building public health inclusivity and best practices.

\section{$\underline{\text { 5.2.5.3 Assets-Based Context }}$}

Importantly, the prototyped methods also communicated participant needs and barriers within the context of creativity and proactive communication, which are themselves significant assets. Even when participants chose to disclose significant needs, losses, or fears, they did so as creators, communicators, poets, artists, advocates, or storytellers. As such, their deficits were not recognizable without or apart from their communicative assets. This benefit may be unique to arts-based data collection, and the potential it represents for more equitable, liberatory, assets-based research—particularly when working with marginalized groups_-warrants further exploration. 


\subsubsection{Attributes of Each Prototype}

This section separates the three sources of arts-based data to discuss findings related to their methods of data elicitation and collection.

\section{$\underline{\text { 5.2.6.1 YDS Intervention }}$}

As noted earlier, written data from YDS was the least aligned with survey results; it also provided the most "outliers" in its content (there were many codes associated with this dataset that did not appear in the others). This is primarily because the data from this population resulted from a variety of prompts given over several months of an ongoing intervention, whereas contest and Wildfire data resulted from far more narrow and specific prompts, within very short timeframes. However, the differences noted in the YDS dataset can also be traced to the unique and traumatizing situation of incarceration, in which considerations of incarceration and justice are likely to occur among participants, as are those of home, family, and loneliness or isolation - all prominent codes in YDS data. Thus, while this dataset was less aligned with surveys and the other two writing sets, it provided extensive information regarding the experience of incarceration among young women, including their priorities (social supports; justice; being heard) and self-identified assets (hope; pride; resilience).

\subsubsection{Writing Contest}

Elicitation of contest data was cost-effective (under $\$ 2,000$, including publication of the resulting anthology); relatively fast (the submission period was March 1-19, 2018); came with built-in dissemination options (publication, reading events, and media/press); 
appealed to multiple city agencies, who assisted its promotion; ${ }^{110}$ highlighted a

community partner (Sarabande Books) instead of researcher activity; and utilized a broad prompt that avoided over-direction while still inviting writers to share violence-related needs, assets, and priorities. The resulting data were broad (this data set utilized the most codes) and, of the three prototyped methods, the contest offered the most extensive information regarding how the target population experienced, perceived, and framed violence. The contest also offers the most straightforward model for replication and future innovation, as it suggests intuitive modifications. For example, the use of a central theme generated entries relevant to a particular research question, and the supportive contest format appealed to arts- and education-based youth networks. In future studies, alternative forms of entries could be solicited, depending on what may be most appealing to the population; these may include themed poetry slams, photography with accompanying descriptions, songs, plays/monologues, essays, etc.

\subsubsection{Pre-existing Art (Wildfire Play)}

The utilization of existing art from the target population (How to Start a Wildfire) as a means of data collection was by far the most time- and cost-effective of the three prototypes, requiring minimal researcher time and no financial investment. ${ }^{111}$ The personal, rich, and detailed nature of the resulting data affirm the value of creative formats, participant autonomy, and self-representation in cultivating trauma-informed (and therefore more inclusive and accessible) spaces that support safe disclosure,

\footnotetext{
${ }^{110}$ Organizations involved in promotion included the Louisville Metro Office of Safe and Healthy Neighborhoods, Metro United Way, Jefferson County Public Schools, Louisville Literary Arts, the Youth Violence Prevention Research Center, and Kentucky Foundation for Women, among others.

${ }^{111}$ In the future, researchers should be prepared to support local, regional, or national arts programming by paying for data that is used in research. By doing so, funds support the generation of knowledge (as with conventional research methods), but local culture is simultaneously supported: creating mutual benefit unique to this arts-based research approach.
} 
participant well-being, and, ultimately, the sharing of knowledge across difference. This prototype also supports the argument in Chapter Two that researchers need not always create new research initiatives - even if such initiatives utilize participatory strategies such as photovoice - in order to learn what a population believes or experiences. This study demonstrated that significant art is often already being created in communities, and that populations have often already answered questions that researchers are asking.

The Wildfire dataset's weakness ${ }^{112}$ lay in its focus on sexual assault and domestic violence, which meant that it did not address other forms of community violence seen in contest and YDS data. However, the play's detail regarding domestic and intimate partner violence was substantial, potentially exceeding that available through interviews - while offering the benefit of not putting survivors through interviews about their traumatic experiences. Future projects may wish to increase data by accessing and analyzing postperformance audience surveys, which are often distributed by performing organizations themselves, and may not require further researcher involvement. These could generate information regarding the extent to which the data of the performance reflected or altered the audience's experiences, beliefs, or perceptions.

\subsubsection{Methods Integration}

Again, it is not necessary that researchers choose between new and conventional methods. The goal of innovation is not to replace existing methods, but to enhance options and practices while challenging the power and privilege often attributed to conventional approaches. Applying critical theory to this study of violence, findings from all sources are best recognized as complementary, with intentional value placed on new

\footnotetext{
${ }^{112}$ The notion of "weakness" with regard to this play is utilized strictly to refer to the analysis at hand.
} 
and diverse knowledges, and intentional suspicion placed on assumptions that surveys render better, more accurate, or more actionable depictions of health "realities."

\subsection{Implications of Findings}

This section draws on the theoretical grounding for this study to present eight implications of the above findings for public health research and practice.

\subsubsection{Implications for Research Design and Health Equity}

Chapter Two argued that the arts-based nature of photovoice was likely responsible for the fact that photovoice study participants were able to "share experiences which would otherwise be difficult to express and process" (Bender et al., 2017, p. 383), ultimately discovering means by which to make "different, safer or more positive disclosures" (p. 47). It further argued that similar benefits were available via creative formats beyond photography, and this study demonstrated that creative writing also offers participants effective strategies for sharing difficult experiences, allowing them to make different, positive disclosures. As a result, these creative formats expand the kinds of knowledge and insight available for public health analysis. For example, the writing contest and Wildfire data sets included insightful detail and information about personal experiences with sexual assault, rape, child abuse, and domestic violence that are unlikely to have emerged in focus groups or one-on-one interviews without significant established trust. ${ }^{113}$ As Napier et al. (2014) asserted, "eliciting clinical responses...should be tempered by an awareness of what cannot be said" (p. 1625).

\footnotetext{
${ }^{113}$ For example, while a young woman may have chosen to reveal during an interview that she had been raped, it is less likely that her disclosure would have been accompanied by detail regarding how this affected her sense of control and self-ownership. The format of poetry appears to have allowed a contest entrant to contextualize her rape by foregrounding these effects - illuminating significant details regarding what it means or requires of public health practice to improve safety or to support trauma recovery.
} 
In addition to expanding what research participants are able to share, this study also demonstrated that creative practices can inform what can or should be studied at all. For example, study participants' poems expanded common definitions of violence while also asserting participants' own identities and priorities; by doing so, they inform how violence is defined or investigated in future studies. The use of creative writing thus offers a promising avenue for reducing oppressive research practices, as it provides a check on researcher assumptions regarding subjects and subject matter.

Further implications for research design and health equity were first highlighted in Chapter Two via three questions: "What do we not know about a given population because individuals are unable to reveal a particular experience or vulnerability? What do we miss when surveys or interviews...demand forthright disclosure, or define experiences differently than participants? And how might failures to gather more complete knowledge be affecting funding and resource decisions, health care access and delivery, and more?" The current study provided some initial answers to these questions; for example, it demonstrated that without arts-based data, we may not have learned how young women in Louisville define violence (as prioritizing D/IPV and trauma; as including loneliness, dismissal, and inaction); we may also have failed to obtain information about particular aspects of the trauma of sexual assault (such as shame, betrayal, or the harmful effects of feeling that one does not own one's body).

In addition, this study conveyed the ability of arts-based data to move beyond forthright disclosure to include contextual and experiential details, such as the harmful effects of loneliness or insecurity, or the critical value to women's health and safety of being and feeling heard. It also demonstrated that surveys' limited definitions of violence 
can lead researchers to miss priority issues, as in the YVPRC survey's failure to convey

exposure to domestic or intimate partner violence. ${ }^{114}$ Similarly, unlike the creative

writing data, the four surveys' data would not have illuminated the potential health

consequences of pervasive sociocultural failures to hear and believe young women, nor

would they have suggested the need for public health investments and research into

shifting related social norms. ${ }^{115}$

In this closing chapter, the researcher would add a fourth question to the three

quoted above: What inequities might we unintentionally perpetuate by failing to provide

means by which research participants can expand researcher definitions, correct

researcher assumptions, contextualize their concerns and experiences, and assert their

own identities and priorities? Similar concerns have motivated participatory action

research (Stanton, 2014; MacDonald, 2012; Wang \& Burris, 1997), which emphasizes

the target population's participation in choosing research goals and practices, and in

\footnotetext{
114 The researcher acknowledges that there are often structural barriers to the inclusion of questions regarding domestic violence, sexual assault, or child abuse, such as institutional and funding restrictions, or mandatory reporting laws that complicate the costs of survey participation. Thus in addition to challenging the strictures of conventional methodologies and epistemologies, researchers may at times have to educate institutions about the dangerously inadequate knowledge produced by research that is circumscribed by convention, assumption, or fear. Researchers can also create and inform institutions about new options for seeking necessary knowledge.

For example, the prevalence of D/IPV nationwide (see NCADV, 2018) - along with its priority among young women in the current study — together indicate the merits of exploring word choices that allow some measurement of D/IPV without implicating a particular respondent and without entailing mandatory reporting. A survey might inquire, for instance, if the respondent has ever witnessed someone they know being hit or harmed by their intimate partner.

${ }^{115}$ When research fails to identify priority issues and potential responses, funding and resource recommendations can be affected, thus inadvertently perpetuating existing concerns or disparities. For example, if researchers rely upon YVPRC survey data to make local funding and resource recommendations regarding youth violence, they will fail to prioritize initiatives and responses related to $\mathrm{D} / \mathrm{IPV}$ - a failure that may ultimately preclude effective strategies for violence prevention and healing. (According to young women in this study, needed local resources include increased education about consent and healthy relationships, increased access to trauma-recovery resources, trauma-informed health care practices and reporting procedures, bystander intervention training that incorporates potential responses to witnessed D/IPV, and meaningful opportunities to share one's story while being heard and believed.)
} 
determining how the community will be represented and depicted. The current study offers an additional model for including and prioritizing the target population's own definitions, contextualizations, and priorities, while simultaneously reducing researcher intrusion, supporting cultural production and expression, and providing new platforms for community self-representation.

These findings make clear that, particularly when dealing with complex, social ecological issues such as violence, research designs that privilege forthright communication, fail to accommodate expanded definitions or interpretations of terms, or rely primarily upon data drawn from predetermined answer options will be inadequate not only as trauma- and culturally-responsive approaches to inquiry (which are fundamental to the advancement of health equity), but also as knowledge-producing mechanisms on which practice can be based. Reliance upon fragmentary, deficient information is likely to create or perpetuate health disparities rather than mitigating them. Thus advancing health equity requires that researchers intentionally expand and develop the methods by which we learn from individuals and communities. ${ }^{116}$

\subsubsection{Implications for Public Health Education and Training}

To be effective, the expansion and development of methods in the field will require that education in public health include: 1) extensive considerations of trauma- and cultural-responsiveness; 2) training in innovation and the value of prototyping; 3) far greater (and more consistent) exposure to creative and arts-based methods and practices;

\footnotetext{
116 The field has increasingly recognized the inadequacy of single-method approaches (see Tariq \& Woodman, 2013, or BSPH, 2018, for example). The particular contributions of the current study include: 1) connecting the use of mixed and multi-method approaches to the issue of health equity; 2) modeling additional methods that are trauma- and culturally-responsive; and 3) identifying some initial ways in which these methods supplement historically-privileged methods while reducing researcher intrusion.
} 
and 4) training in critical and intersectional theories. As demonstrated in Chapters One and Two, the first and last of these are fundamental for effective and equitable public health research and practice. Together, they frame the importance of questioning assumptions, maintaining awareness of power structures and dynamics, cultivating communicative options, and valuing diverse knowledges. ${ }^{117}$

It is worth noting that effective pedagogy related to these factors will need to model them. Thus public health educators should be prepared to: bring trauma- and culturally-responsive practices into the classroom; acknowledge and critique the power structures and dynamics inherent in higher education; explicitly value diverse forms of knowledges and meaning-making; and use and allow multiple communication strategies. The current study therefore has implications for changes to teaching practices, competencies, expectations, and training opportunities. ${ }^{118}$

\subsubsection{Implications for Social Ecological Thinking}

Although social ecological approaches already include "culture" as a level, the ability for varied communications practices to differently elicit, value, or obscure knowledge affirms that research practices must themselves be recognized as aspects of culture, and as significant determinants of health and health equity.

Similarly, the role of hermeneutic theory in the current study indicates that more emphasis may need to be placed on perceptions and meaning-making processes as aspects of culture that are also themselves determinants of behaviors, resources, health,

\footnotetext{
${ }^{117}$ Notably, these fit within the Council on Education for Public Health's (CEPH) "MPH Foundational Competencies," particularly number six ("Discuss the means by which structural bias, social inequities and racism undermine health...") and all "Communications" competencies (18 through 20). See CEPH, 2016, p. 17.

${ }^{118}$ Critical pedagogy figures as a potential model for the increased modeling of new educational approaches, both in classrooms and in public health practice in the community. This is discussed in Section 5.5 .
} 
and well-being. For example, in the current study, those writers for whom experiences of violence were attributable to a hopelessly evil society may have higher risks for depression or exhibit different health behaviors than those who perceived violence as a challenge to be overcome. These are differences of perception, meaning, and valuation that can effect disparate results despite potential similarities in lived experience. Thus an examination of health determinants that fails to elicit, analyze, or respond to meaningmaking processes - and their determinants - is ultimately inadequate for understanding and responding to health disparities.

As Little (2008) argued, it is necessary that meanings and meaning-making processes — traditionally studied using hermeneutic analysis ${ }^{119}$ — be joined with traditional causal research in social sciences in order to illuminate the "range of causal mechanisms" (n.p.) leading to health or its lack. In other words, expanding communicative options in public health inquiry will not only increase access, disclosure, and equity (as already discussed), but also offer mechanisms by which investigators can learn about events and their attributed meanings. To support this examination of meanings and meaning-making, public health education and training will need to include some measure of hermeneutic theory and analysis, ideally alongside education in traumaand cultural-responsiveness.

\footnotetext{
${ }^{119}$ Hermeneutics as a practice of analysis centers the examination of 1) the meaning of an action for the actor themself; 2) how that action interacts with other actions (whether those of immediate others or of socio-culturo-historical contexts); and 3) the meaning assigned to that action by others, whether those others are immediately present or historically or culturally distant.
} 


\subsubsection{Implications for Needs Assessments}

Moving to immediate applications, the tested prototypes offer particular promise with regard to qualitative data collection and needs assessments. Standard community needs assessments typically seek to identify communities' health-related needs and problems, priorities, and existing assets—including how the latter may support action steps moving forward (Wright, Williams, \& Wilkinson, 1998; Cavanagh \& Chadwick, 2005; Burdine \& Wendel, 2007; Community Tool Box, 2018). In addition, particularly when researchers are outsiders to the researched community, needs assessments often include questions about how community members understand or define the concept of health itself (see De La Cancela et al., 2016). Given the prototypes' particular adeptness at relaying these types of information using an inclusive, culturally-responsive, strengthsbased approach, they hold great promise for uptake in needs assessment practices moving forward.

\subsubsection{Implications for Ethical Considerations}

In addition to aiding disclosure — and thereby improving study participation, access, and data — methods inclusive of art and creativity also offer the potential for direct health benefits. ${ }^{120}$ If research practices themselves can directly benefit participants' health, then researchers' choice of methodologies must be recognized as including a choice to offer or withhold such benefits. In this sense, research design involves ethical considerations that may previously have been left undiscussed and unaddressed.

\footnotetext{
${ }^{120}$ As noted throughout the dissertation, these include multiple benefits associated with expressive writing (Frattaroli, 2006; Pennebaker, 1999; Travagin et al., 2015); increased sense of meaning and control associated with placing experiences into a narrative (Goodson \& Gill, 2014; Greenbaum \& Javdani, 2016; Chandler et al., 2015); and reductions in isolation and stigma resulting from communicating and feeling heard (Alvarez \& Mearns, 2012). In developing best practices in public health inquiry, these benefits will need to be further explored and prioritized; see section 5.4.3.
} 


\subsubsection{Implications for Health Communications}

When researchers assume that new research questions require new entries into a given population, they assume that the target population was not already effectively expressing and documenting relevant information. These assumptions are grounded in the privileging of dominant scientific forms of information and communication, and they can perpetuate the practice of over-researching under-resourced communities. A potential benefit of increased utilization of arts-based data in public health—such as that demonstrated by the current study — is that health researchers and practitioners would become better attuned to communities' arts and cultural practices, and thus better prepared to identify and respond to how a given research question is already being answered via various forms of cultural and artistic expression.

This awareness of community arts and cultural practices could also improve health promotion and policy advocacy efforts within public health by incorporating art and stories from the affected community. This would increase self-representation by the community while supporting awareness, empathy, and social connection. Although practitioners do regularly incorporate artwork into advocacy and awareness campaigns (Bailey, 2015; 'The Art of Public Health,' 2012; WHO, 2009), an ongoing, intentional connection to a target population's own art is likely to render the integration of art into such campaigns more specific, accessible, and intuitive. ${ }^{121}$

\footnotetext{
${ }^{121}$ It should go without saying that all artists should be compensated for the use of their work. Assumptions that local artists will work for free or for minimal payment are condescending; they also perpetuate the devaluing of local community production rather than recognizing it as worthy of support.
} 


\subsubsection{Implications for Interprofessional Communications}

The ability for arts-based communications to transmit meaning and increase empathy has implications for health communications beyond data collection and health promotion—including research dissemination, policy advocacy, and public health pedagogy. For example, how might arts-based approaches improve dissemination and reception of study results, or of presentations to leaders and policymakers? And, as discussed above, how might arts-based approaches enhance public health classrooms, or health care provider training? It is not only possible but perhaps imperative that researchers become strategically creative about the ways in which we listen, speak, depict, and discover.

\subsubsection{Implications for Research Funding}

This study demonstrated that creative practices-including existing population art — can offer productive, trauma- and culturally-responsive sources of public health data that are assets-based, conducive to peer education, and productive of highly disseminatable community self-representations. Given these benefits, it is reasonable to expect that some funds currently allotted for public health needs assessments, surveys, focus groups, and interviews should instead be channeled to local arts programs, events, or initiatives - particularly among marginalized populations. Knight (2014) noted the "need for a more politically active public health practice, which includes interventions in...the cultural realm, and prioritizes empowerment of individuals and communities that experience social disadvantage" (p. 194). ${ }^{122}$ By directing funding to arts-inclusive

\footnotetext{
${ }^{122}$ Knight also noted that "[p]ublic health must...use its authority to legitimize alternate sources of knowledge and prioritize community-based approaches to assessment" (2014, p. 194).
} 
practices, public health could support local, valued cultural practices based not on charity or even (strictly) on health promotion goals, but on the recognition of these practices as the powerful sources of meaning and knowledge that they have always been. As Napier et al. (2014) argued, "All possible avenues to understand and nourish wellbeing should become the highest health-care priority and publicly supported and funded at least as much as new biomedical research" (p. 1633).

Shifting research funding to arts initiatives would also undergird public health's incipient investment in the role of culture in health (Singer, Dressler \& George, 2016; Napier et al., 2014; Unger, 2011), and would likely improve relationships between communities and research institutions by cultivating reciprocity and mutual understanding. Notably, funding for arts-inclusive research may also generate its own health benefits, as general increased exposure and participation in the arts has been linked to reduced chronic pain, improvements in quality of life measures, greater social cohesion, and increased community development (Stuckey \& Nobel, 2010; Hanna, Rollins, \& Lewis, 2017; Markusen \& Gadwa, 2010).

\subsection{Limitations and Future Directions}

Previous methodological research related to photovoice established the value of creative, image-based formats for increasing participation, access, retention, data, and self-representation, particularly among marginalized populations. The current study built upon that research to test new creative formats, new methods of eliciting art, and benefits beyond those typically discussed in photovoice literature. The previous sections discussed the contributions of these efforts; the following section discusses the limitations of the current study—incorporating recommendations for future research. 


\subsubsection{Data Analysis}

As with qualitative data in general, the process of codifying poems presented subjective choices that may have produced different results had they been made by or in collaboration with other researchers. In particular, poetic strategies such as anaphora and refrains, which generate repetition, presented unusual quandaries in that they required the researcher to choose whether to document a single code each time it was mentioned, or to document a given poem in which it appeared as one single (if emphatic) use of the code. Ultimately, the researcher determined that a code should be documented each time the concept was presented in a new way, or with a new effect, response, or related concerneven if these occurred in the same poem. So for example, the code "gender expectations" was documented multiple times in one poem in which a young woman described being robbed. Each code's appearance corresponds with separate illustrations of gender expectations: taught not to make a scene, taught not to say no, trained to be unassertive,

and taught to acquiesce. While this strategy addressed the issue of repetition, it is possible that another researcher may have differently identified reoccurrences, leading to different conclusions.

To manage the inevitable variation in researchers' coding choices and interpretations, care was taken in this study to systematize the coding process. This was accomplished by setting inclusion and exclusion parameters and including multiple rounds of coding in order to render the most reliable findings.

\subsubsection{Creative Writing}

As a pilot study of new methods, the current study focused narrowly on creative writing from three potential source types (intervention, call for submissions, existing 
community art). While this produced extensive data, findings do not reflect creative forms of communication beyond writing. As a result, the current study does not challenge the textocentricity of conventional methods of data collection; as such, it could not accommodate individuals who were not verbal, or who lacked access to literacy in the English language. In addition, although the current study demonstrates the value of creative writing in public health communication, it is clear that nonverbal communicative forms such as dance, painting, or music composition would require different analytical and translational processes than creative writing. These processes will themselves require continued development, as well as subsequent teaching and training as part of research education.

Nevertheless, the choice of creative writing for the pilot study was intentional, based upon four factors: 1) an image-based method, photovoice, has already been widely studied — warranting exploration of alternate formats; 2) tools and space for writing are often more accessible than music or art supplies, rendering creative writing a promising mechanism for accessible creative practice; 3) the text-based nature of creative writing renders its process of analysis more similar to that of most qualitative data than image- or movement-based works; and 4) the researcher's background and expertise in creative writing supported attentive and robust analysis. It is hoped that this pilot will stimulate further use and exploration of creative writing in its multiple forms (song, spoken word, short stories, etc.), while also emphasizing the need for ongoing innovation with multiple forms of communication.

For future research and theoretical development, it is important to note that this study's focus on creative writing calls attention to the inherently created and creative 
nature of health realities by acknowledging them as sources and effects of story-making, storytelling, and revision. By doing so, this study not only affirms the need for critical epistemological reflection in health assessment and planning (challenging positivist assumptions), but also affirms the inescapable role of narrative and creativity in perpetuating or challenging existing stories about a population's needs, assets, values, priorities, and life experiences. Goodson \& Gill (2014) argued that "re-storying is a key to social imagination and collective transformative action" (p. 98); given public health's imperative to take transformative action related to health equity, this study's emphases on cultural and creative inquiry provide material for further theoretical deliberation and continued innovation.

\subsubsection{Direct Health Benefits}

As noted throughout this dissertation, the literature on expressive writing, narrative, and arts in health offer extensive indications that arts-based methods can generate direct health benefits to participants. However, this study did not include an evaluation of these benefits for participants; it is thus not possible to demonstrate that participants indeed experienced direct health improvements as a result of writing, submitting, performing, or publishing their creative works. While this presents a limitation of the current study, the choice not to include this evaluation supported other goals of the study, including minimizing researcher intrusion and preventing test bias. Future studies may wish to include pre/post surveys or follow-up interviews or focus groups to evaluate whether and to what extent participants experienced health benefits as a result of participating in creative activity. 


\subsubsection{Demographic Analysis}

As noted in Chapter Four, analyzing demographic disparities in the written data was beyond the scope of the current project. As a result, findings from these data sets do not incorporate potential inequities based on race, age, sexual orientation, or other factors. Given that demographic data were available for many YDS and contest participants, future research may include analysis of themes by demographic group as a means of noting disparities in concerns, priorities, or assets. Meanwhile, the differential affordances and limitations of all utilized methods (surveys and creative writing) underlines another value of incorporating multiple methods in research design: desired variables do not have to be obtained via every method.

As Chapter Four noted, artists typically wish to be credited for their work, which precludes the anonymity that supports the sharing of demographic data in other methods. This lack of anonymity creates a dilemma unique to arts-based research; future researchers and artists should together explore and evaluate the benefits and risks of collecting or going without demographic information. Results of these mutual evaluations are likely to be different for each initiative, research question, and population.

\subsubsection{Translating Data}

Finally, in testing the ability of new methods to offer trauma- and culturallyresponsive approaches to researching difficult topics, this study actually translated the diverse knowledges it values into the types of knowledge privileged by the scientific community. ${ }^{123}$ (This is most immediately seen in Chapter Four's translation of poetry into

\footnotetext{
${ }^{123}$ This is regularly done in the analysis of any qualitative data, such as interview and focus group transcripts, in which quantifying, replicating, and triangulating individuals' communications is "known" to render trustworthiness the data did not previously possess.
} 
numbers, charts, and percentages.) As a result, the study risks perpetuating the knowledge hierarchy it critiques: elevating arts-based forms of communication less for their inherent value than for their ability to be transformed into what the scientific community has already recognized and legitimized.

The author was aware of this risk, yet finds the concept of translation to be useful in highlighting the study's ultimate goal of supporting more effective and equitable communication. After all, the practice of translation is employed when communication is needed across difference, time, or geography; it relies on the notion that a meaning exists in one language, to which access must or should be increased via translation. Of course, as Susan Sontag (2007) observed, "translation is basically an impossible task, if what is meant is that the translator is able to take up the text of an author written in one language and deliver it, intact, without loss, into another language" (p. 158). The impossibility of such delivery implies ethical choices as the translator alters original works, ${ }^{124}$ and the author took these choices seriously when codifying and re-presenting young women's knowledge and art. The risk that this process could diminish young women's words by rendering them in scientific terms was balanced by the recognition that translation "is an opportunity to allow a foreign tongue to influence and modify the language into which a work is being translated" (Sontag, 2007, p. 176). In other words, this pilot study of prototyped creative methods was conducted with the hope that the translations it required will increase the presence and use in scientific discourse of: creative communications; metaphors (such as "wildfire") that help extend individual, biomedical experience into

\footnotetext{
${ }^{124}$ Sontag rendered this act synonymous with the act of re-creation. For her, the task of ethical translation is made all the more daunting by the recognition that any process of adapting knowledge for new audiences entails a reworking of the original that inevitably alters its meaning to some extent.
} 
social ecological shifts and responsibilities; and firsthand accounts by under-represented individuals which, given their immediacy, imagery, and linguistic force, will ideally be increasingly recognized as outperforming numbers as depictions of human reality. ${ }^{125}$

\subsection{Conclusion}

As noted in Chapter Two, the field of public health has struggled in its advancement of health equity, its facilitation of policy advocacy, and its movement of health promotion beyond individual behaviors to the outer levels of the social ecological model. However, its theoretical commitments to these efforts are pervasive throughout the literature (Cohen \& Marshall, 2015). This disjunction between the field's commitments and actual practices has created an urgent window of opportunity to develop innovative strategies that combine existing public health methods with exploratory forms that reflect an interdisciplinary examination of knowledge production, trauma, stigma, communication, and cultural expression. Utilizing the Public Health Innovation Model, this study developed three prototypes that drew on the benefits and limitations of photovoice to examine new possibilities for public health inquiry and data collection. It is hoped is that the findings - as well as the modeled process of innovating and prototyping methods-will support ongoing efforts to improve trauma responsiveness, cultural responsiveness, and the field's ability to challenge epistemological assumptions - all of which are required to advance public health and health equity.

\footnotetext{
${ }^{125}$ Meanwhile, the researcher highly recommends the original sources of data. An anthology of poems resulting from the writing contest is available via Sarabande Books at: http://www.sarabandebooks.org/swlanthologies/vol-10. One Day I'll Rise, an anthology of poems from girls in YDS in 2018, is also available from Sarabande Books at: http://www.sarabandebooks.org/swl-anthologies/one-day-ill-rise-sarabandewriting-labs-vol-xi.
} 


\section{REFERENCES}

Aburn, G., Gott, M., \& Hoare, K. (2016). What is resilience? An Integrative Review of the empirical literature. Journal of Advanced Nursing.

ACEs Too High. (2017). ACEs Science 101. Retrieved from https://acestoohigh.com/aces-101/

Adibi, H. (2014). Health: Its Implications within the Biomedical and Social Models of Health - a Critical Review. Multidisciplinary Journals in Science and Technology, Journal of Selected Areas in Health Informatics, 4(2), 16-23. Retrieved from http://www.cyberjournals.com/Papers/Feb2014/09.pdf

Al-Azri, N. H. (2012). Do we need a new medical paradigm? Oman Medical Journal, 27(3), 256-7. http://doi.org/10.5001/omj.2012.60

Alparone, F. R., Pagliaro, S., \& Rizzo, I. (2015). The words to tell their own pain: Linguistic markers of cognitive reappraisal in mediating benefits of expressive writing. Journal of Social and Clinical Psychology. http://doi.org/10.1521/jscp.2015.34.6.495

Alvarez, N., \& Mearns, J. (2014). The benefits of writing and performing in the spoken word poetry community. Arts in Psychotherapy, 41(3), 263-268.

http://doi.org/10.1016/j.aip.2014.03.004 
Anda, R. F., \& Felitti, V. J. (2012). Adverse Childhood Experiences and their Relationship to Adult Well-being and Disease : Turning gold into lead. Retrieved from https://www.thenationalcouncil.org/wp-content/uploads/2012/11/Natl-CouncilWebinar-8-2012.pdf

'The Art of Public Health': Pictures meant to save a thousand lives. (2012). Retrieved from https://news.yale.edu/2012/04/11/art-public-health-pictures-meant-savethousand-lives

Ashcroft, R., \& Van Katwyk, T. (2016). An Examination of the Biomedical Paradigm: A View of Social Work. Social Work in Public Health, 31(3), 140-152. http://doi.org/10.1080/19371918.2015.1087918

Baker, L., Mohamed, S., Straatman, A.L., \& Tabibi, J. (2016). Building a traumainformed health promotion community of practice. London, ON.

Bailey, C. (2015). Art in Health Education. Retrieved from http://seedglobalhealth.org/art-in-health-education/\#.XC1Oci2ZOfU

Ball, A., \& Mackrain, M. (2008). Devereux Adult Resilience Survey (DARS). Professional Psychology Trainee. Retrieved from www.CenterForResilientChildren.org

Barad, K. M. (2007). Meeting the universe halfway: quantum physics and the entanglement of matter and meaning.

Bauman, Z. (1978). Hermeneutics and social science. London: Routledge.

Beavis, A. S. W., Hojjati, A., Kassam, A., Choudhury, D., Fraser, M., Masching, R., \& Nixon, S. A. (2015). What all students in healthcare training programs should learn to increase health equity: perspectives on postcolonialism and the health of 
Aboriginal Peoples in Canada. BMC Medical Education, 15, 155.

http://doi.org/10.1186/s12909-015-0442-y

Bender, K., Barman-Adhikari, A., DeChants, J., Haffejee, B., Anyon, Y., Begun, S., ...

Dunn, K. (2017). Asking for Change: Feasibility, acceptability, and preliminary

outcomes of a manualized photovoice intervention with youth experiencing

homelessness. Children and Youth Services Review, 81(August), 379-389.

http://doi.org/10.1016/j.childyouth.2017.08.028

Benjet, C., Bromet, E., Karam, E. G., Kessler, R. C., McLaughlin, K. A., Ruscio, A. M., ... Koenen, K. C. (2016). The epidemiology of traumatic event exposure worldwide: results from the World Mental Health Survey Consortium. Psychological Medicine, 46(2), 327-43. http://doi.org/10.1017/S0033291715001981

Bergold, J., \& Thomas, S. (2012). Participatory Research Methods: A Methodological Approach in Motion. Forum: Qualitative Social Research, 13(1). Retrieved from http://www.qualitative-research.net/index.php/fqs/article/view/1801/3334

Bernhardt, J. M. (2010). Communication at the Core of Effective Public Health Communication at the Core of Effective Public Health. American Journal of Public Health, 94(12), 2051-2053. http://doi.org/10.2105/AJPH.94.12.2051

Bhattacharya, K. (2009). Othering Research, Researching the Other: De/Colonizing Approaches to Qualitative Inquiry (pp. 105-150). Springer, Dordrecht. http://doi.org/10.1007/978-1-4020-9628-0_3

Black, N., \& Donald, A. (2001). Evidence based policy: proceed with care. Bmj, 323(7307), 275-279. http://doi.org/10.1136/bmj.323.7307.275 
Bloom, S. L. (2018). The Sanctuary Model. Retrieved from http://sanctuaryweb.com/PublicHealth/TraumaInformedSystems/SAMHSAEssentialElementsTrauma-informedCare.aspx

Bowen, E. A., \& Murshid, N. S. (2016). Trauma-informed social policy: A conceptual framework for policy analysis and advocacy. American Journal of Public Health, 106(2), 223-229. http://doi.org/10.2105/AJPH.2015.302970

Brown, L., \& Strega, S. (2005). Trangressive Possibilities. In L. Brown \& S. Strega (Eds.), Research as Resistance (pp. 1-18). Toronto, ON: Canadian Scholars' Press. Brownell, S. E., Price, J. V, \& Steinman, L. (2013). Science Communication to the General Public: Why We Need to Teach Undergraduate and Graduate Students this Skill as Part of Their Formal Scientific Training. Journal of Undergraduate Neuroscience Education : JUNE : A Publication of FUN, Faculty for Undergraduate Neuroscience, 12(1), E6-E10. Retrieved from http://www.pubmedcentral.nih.gov/articlerender.fcgi?artid=3852879\&tool=pmcentr ez\&rendertype $=$ abstract

Brownlee, T., \& Lee, K. (2018). Chapter 27. Cultural Competence in a Multicultural World | Section 7. Building Culturally Competent Organizations | Main Section | Community Tool Box. Retrieved from https://ctb.ku.edu/en/table-ofcontents/culture/cultural-competence/culturally-competent-organizations/main Buckley, M. E. (2000). Beyond the rhetoric of empowerment: A critical analysis of gender, participation and empowerment. Dalhousie University. Retrieved from http://www.collectionscanada.gc.ca/obj/s4/f2/dsk2/ftp03/MQ57274.pdf 
Budig, K., Diez, J., Conde, P., Sastre, M., Hernán, M., \& Franco, M. (2018). Photovoice and empowerment: Evaluating the transformative potential of a participatory action research project. BMC Public Health, 18(1), 1-9. http://doi.org/10.1186/s12889018-5335-7

Bygren, L. O., Konlaan, B. B., \& Johansson, S. E. (n.d.). Attendance at cultural events, reading books or periodicals, and making music or singing in a choir as determinants for survival: Swedish interview survey of living conditions. BMJ (Clinical Research Ed.), 313(7072), 1577-80. Retrieved from http://www.ncbi.nlm.nih.gov/pubmed/8990990

Camic, P. M. (2008). Playing in the mud: Health psychology, the arts and creative approaches to health care. Journal of Health Psychology, 13(2), 287-298. http://doi.org/10.1177/1359105307086698

Carson, D., Gilmore, A., Perry, C., \& Gronhaug, K. (2001). Qualitative Marketing Research. London: Sage.

Catalani, C., \& Minkler, M. (2010). Photovoice: A Review of the Literature in Health and Public Health. Health Education \& Behavior, 37(3), 424-451. http://doi.org/10.1177/1090198109342084

Cavanagh, S., \& Chadwick, K. (2005). Health needs assessment: A practical guide. London. Retrieved from www.nice.org.uk

CDC. (2014). Practical Strategies for Culturally Competent Evaluation Guide Retrieved from https://www.cdc.gov/dhdsp/docs/cultural_competence_guide.pdf

CDC. (2016). University of Louisville Youth Violence Prevention Research Center. Atlanta, GA. Retrieved from www.pridepeaceprevention.org 
Center for Health Equity. (2018). Center for Health Equity: Our Vision. Retrieved from https://louisvilleky.gov/government/center-health-equity

Chandler, G. E., Roberts, S. J., \& Chiodo, L. (2015). Resilience Intervention for Young Adults With Adverse Childhood Experiences. Journal of the American Psychiatric Nurses Association, 21(6), 406-416. http://doi.org/10.1177/1078390315620609

Cho, S., Crenshaw, K. W., \& McCall, L. (2013). Toward a Field of Intersectionality Studies: Theory, Applications, and Praxis. Signs: Journal of Women in Culture and Society, 38(4), 785-810. http://doi.org/10.1086/669608

Christakis, N. A., \& Fowler, J. H. (2009). Connected: The Surprising Power of Our Social Networks and How They Shape Our Lives (1st ed.). New York, NY: Little, Brown and Company.

Clift, S. (2012). Creative arts as a public health resource: Moving from practice-based research to evidence-based practice. Perspectives in Public Health, 132(3), 120127. http://doi.org/10.1177/1757913912442269

Clift, S., \& Camic, P. M. (2016). Oxford textbook of creative arts, health, and wellbeing : international perspectives on practice, policy and research (First). Oxford: Oxford University Press.

Coad, J. (2007). Using art-based techniques in engaging children and young people in health care consultations and/or research. Journal of Research in Nursing, 12(5), 487-497. http://doi.org/10.1177/1744987107081250

Coemans, S., \& Hannes, K. (2017). Researchers under the spell of the arts: Two decades of using arts-based methods in community-based inquiry with vulnerable 
populations. Educational Research Review, 22, 34-49.

http://doi.org/10.1016/j.edurev.2017.08.003

Cohen, B. E., \& Marshall, S. G. (2017). Does public health advocacy seek to redress health inequities? A scoping review. Health and Social Care in the Community, 25(2), 309-328. http://doi.org/10.1111/hsc.12320

Community Tool Box. (2018). Chapter 3 Section 7. Conducting Needs Assessment Surveys. In C. Holt, S. Fawcett, V. Francisco, J. Schultz, B. Berkowitz, \& T. Wolff (Eds.), Community Tool Box. Lawrence, KS: Center for Community Health and Development at the University of Kansas. Retrieved from https://ctb.ku.edu/en/table-of-contents/assessment/assessing-community-needs-andresources/conducting-needs-assessment-surveys/main

Congress Lowitja. (2014). Culture Identity and Health. Retrieved from https://www.lowitja.org.au/congress-2014/sessions/culture-identity-health

Council on Education for Public Health (CEPH). (2016). Accreditation Criteria: Schools of Public Health and Public Health Programs. Silver Spring, MD. Retrieved from www.ceph.org

Cox, S. M., \& Boydell, K. M. (2015). Ethical Issues in Arts-Based Health Research. In S. Clift \& P. M. Camic (Eds.), Oxford Textbook of Creative Arts, Health, and Wellbeing: International perspectives on practice, policy and research (pp. 83-89). New York, NY: Oxford University Press.

Crenshaw, K. (2018). Demarginalizing the intersection of race and sex: A black feminist critique of antidiscrimination doctrine, feminist theory, and antiracist politics [1989]. 
In Feminist Legal Theory: Readings in Law and Gender.

http://doi.org/10.4324/9780429500480

Curtis, S., Bryce, H., \& Treloar, C. (1999). Action Research: Changing the Paradigm for Health Psychology Researchers. In Qualitative Health Psychology: Theories and Methods (pp. 202-2017). Sage Publications.

Dahlberg, L., Toal, S., Swahn, M., \& Behrens, C. (2005). Measuring Violence-Related Attitudes, Behaviors, and Influences Among Youths: A Compendium of Assessment Tools (Second Edition). Atlanta, GA. Retrieved from https://www.cdc.gov/violenceprevention/pdf/yv_compendium.pdf

Dahm, J. (2014). Commentary: Decolonizing Healthcare. Retrieved from https://www.mcgilldaily.com/2014/09/decolonizing-healthcare/

Dalgaard, N. T., \& Montgomery, E. (2015). Disclosure and silencing: A systematic review of the literature on patterns of trauma communication in refugee families. Transcultural Psychiatry, 52(5), 579-593. http://doi.org/10.1177/1363461514568442

Davies, C., Pescud, M., Anwar-McHenry, J., \& Wright, P. (2016). Arts, public health and the National Arts and Health Framework: a lexicon for health professionals. Australian and New Zealand Journal of Public Health, 40(4), 304-306. http://doi.org/10.1111/1753-6405.12545

De La Cancela, V., Chin, J. L., \& Jenkins, Y. (1998). Community Health Psychology: Empowerment for Diverse Communities. Routledge. New York, NY. 
Delgado, M. (2015). Urban youth and photovoice: Visual ethnography in action. Urban youth and photovoice: Visual ethnography in action. http://doi.org/10.1080/17441692.2016.1169308

Desyllas, M. C. (2014). Using photovoice with sex workers: The power of art, agency and resistance. Qualitative Social Work, 13(4), 477-501. http://doi.org/10.1177/1473325013496596

Dickson-Hughes, G., Dimopoulos, L., Dropsy, A., Graham, C., Horvat, L., Manabat, C., ... Summers, M. (2009). Cultural responsiveness framework: Guidelines for Victorian health services. Melbourne, Victoria. Retrieved from http://www.health.vic.gov.au/cald

Duncombe, S., Perlov, G., Lamber, S., \& Halford, S. J. (2017). Assessing the Impact of Artistic Activism. New York, NY.

Edge, S., Newbold, K. B., \& Mckeary, M. (2014). Social Science \& Medicine Exploring socio-cultural factors that mediate, facilitate , \& constrain the health and empowerment of refugee youth. Social Science \& Medicine, 117, 34-41. http://doi.org/10.1016/j.socscimed.2014.07.025

EPISCenter. (n.d.). The Child PTSD Symptom Scale for DSM-V (CPSS-V SR) Psychometric Properties Summary. University Park, PA . Retrieved from http://www.episcenter.psu.edu/sites/default/files/CPSS-V Scoring \%26 Psychometrics.pdf

Evans-Agnew, R. A., \& Rosemberg, M.-A. S. (2016). Questioning Photovoice Research. Qualitative Health Research, 26(8), 1019-1030. http://doi.org/10.1177/1049732315624223 
Ferreira, M. P., \& Gendron, F. (2011). Community-based participatory research with traditional and indigenous communities of the Americas: Historical context and future directions. International Journal of Critical Pedagogy (Vol. 3). Retrieved from http://libjournal.uncg.edu/ijcp/article/viewFile/254/119

Ferris, M. (2012). Social Connectedness and Health. Saint Paul, MN.

Feyerabend, P. K. (2011). The Tyranny of Science. Polity Press. Retrieved from https://www.amazon.com/Tyranny-Science-Paul-Feyerabend-2011-0425/dp/B01FIYTTNG

Fine, M., \& Torre, M. E. (2006). Bearing Witness: Methods for Researching Oppression and Resistance-A Textbook for Critical Research. Social Justice Research, 19(2), 194-200. http://doi.org/10.1007/s11211-006-0001-0

Fitzgerald, M., \& Shipman, K. (n.d.). Child PTSD Symptom Scale (CPSS) Scoring Clinical Cutoff. Retrieved from https://coactcolorado.org/site_media/media/servee_documents/CPSS_Scoring.pdf

Fleckman, J. M., Corso, M. D., Ramirez, S., Begalieva, M., Caron, R. M., Bereknyei, S., ... Johnson, C. C. (2015). Intercultural competency in public health: a call for action to incorporate training into public health education. Frontiers in Public Health, (September), 1-7. http://doi.org/10.3389/fpubh.2015.00210

Ford, C. L., \& Airhihenbuwa, C. O. (2010). Critical race theory, race equity, and public health: Toward antiracism praxis. American Journal of Public Health, 100(SUPPL. 1), 693-698. http://doi.org/10.2105/AJPH.2009.171058

Foucault, M., \& Gordon, C. (1980). Power/knowledge : selected interviews and other writings, 1972-1977. Pantheon Books. 
Fox, N. J. (2012). Creativity and health : An anti-humanist reflection, 49-50. http://doi.org/10.1177/1363459312464074

Frattaroli, J. (2006). Experimental disclosure and its moderators: A meta-analysis. Psychological Bulletin, 132(6), 823-865. http://doi.org/10.1037/00332909.132.6.823

Freire, P. (2005). Pedagogy of the Oppressed (30th Anniv). New York, NY: continuum.

Geuss, R. (1981). The idea of a critical theory: Habermas and the Frankfurt school. Cambridge University Press.

Gill, G. K., \& Babacan, H. (2012). Developing a cultural responsiveness framework in healthcare systems: an Australian example. Diversity \& Equality in Health and Care, (9), 45-55.

Glowicki, M. (2014, September 20). Zones of Hope initiative launches. Courier Journal. Louis ville, KY. Retrieved from https://www.courierjournal.com/story/news/local/2014/09/20/zones-hope-initiative-launches/15946961/

Golden, T. (2017). Subalternity in Juvenile Justice: Gendered Oppression and the Rhetoric of Reform. Reflections: Public Rhetoric, Civic Writing and Service Learning, 17(1), 156-188.

Good Therapy. (2018). Adverse Childhood Experience (ACE) Questionnaire. Retrieved from https://www.goodtherapy.org/blog/psychpedia/ace-questionnaire

Goodson, I., \& Gill, S. (2014). Critical Narrative as Pedagogy. London: Bloomsbury.

Greenbaum, C. A., \& Javdani, S. (2017). Expressive writing intervention promotes resilience among juvenile justice-involved youth. Children and Youth Services Review, 73, 220-229. http://doi.org/10.1016/j.childyouth.2016.11.034 
Gronholm, P. C., Henderson, C., Deb, T., \& Thornicroft, G. (2017). Interventions to reduce discrimination and stigma: the state of the art. Social Psychiatry and Psychiatric Epidemiology, 52(3), 249-258. http://doi.org/10.1007/s00127-017-13419

Hand, J., \& Golden, T. (2018). Arts, Culture, and Community Mental Health. Community Development Innovation Review, 13(1).

Hanna, G., Rollins, J., \& Lewis, L. (2017). Arts in Medicine Literature Review. Seattle, WA.

Harley, A. (2012). Picturing reality: Power, ethics, and politics in using photovoice. International Journal of Qualitative Methods, 11(4), 320-339. Retrieved from http://ejournals.library.ualberta.ca/index.php/IJQM/article/view/6030/14341

Horkheimer, M. (1982). Critical theory: selected essays. Continuum Pub. Corp.

Houghton, T. (2011). Does positivism really "work" in the social sciences?, 1-5. Retrieved from http://www.e-ir.info/2011/09/26/does-positivism-really-'work'-inthe-social-sciences/

House, E. R. (1980). Evaluating with validity. Sage Publications.

Huen, J. M. Y., Ip, B. Y. T., Ho, S. M. Y., \& Yip, P. S. F. (2015). Hope and Hopelessness: The Role of Hope in Buffering the Impact of Hopelessness on Suicidal Ideation. PloS One, 10(6), e0130073. http://doi.org/10.1371/journal.pone.0130073

Hughes, J. A., \& Sharrock, W. W. (Wes W. . (1997). The philosophy of social research. Longman. Retrieved from 
https://books.google.com/books/about/The_Philosophy_of_Social_Research.html?id $=$ j0y9SAA

Ings, R., Crane, N., \& Cameron, M. (2012). Be Creative Be Well. London, England.

Israel, B. A., Schulz, A. J., Parker, E. A., \& Becker, A. B. (2001). Community-based participatory research: Policy recommendations for promoting a partnership approach in health research. Education for Health, 14(2), 182-197. http://doi.org/10.1080/13576280110051055

Jacobson, N., Butterill, D., \& Goering, P. (2004). Organizational Factors that Influence University-Based Researchers' Engagement in Knowledge Transfer Activities. Science Communication, 25(3), 246-259. http://doi.org/10.1177/1075547003262038

Janßen, C., Sauter, S., \& Kowalski, C. (2012). The influence of social determinants on the use of prevention and health promotion services: Results of a systematic literature review. Psycho-Social Medicine, 9, Doc07. http://doi.org/10.3205/psm000085

Johns Hopkins Bloomberg School of Public Health (BSPH). (2018). Why Mixed Methods? Retrieved from https://www.jhsph.edu/academics/trainingprograms/mixed-methods-training-program-for-the-health-sciences/about-theprogram/why-mixed-methods/index.html

Johnston, G. (2016). Champions for social change: Photovoice ethics in practice and ‘false hopes' for policy and social change. Global Public Health, 11(5-6), 799-811. Johnson, N. (2016). Trauma-Informed Evaluation. St. Paul, MN. Retrieved from https://www.wilder.org/Wilder-Research/Publications/Studies/Program Evaluation 
and Research Tips/Trauma-Informed Evaluation - Tip Sheet for Collecting Information.pdf

Jones, M. (2017, April). Youth violence: The Secret Geography of Louisville. LEO Weekly.

Jordan, J. (2004). Beyond Belief? Criminal Justice, 4(1), 29-59. http://doi.org/10.1177/1466802504042222

Kalmakis, K. A., \& Chandler, G. E. (2015). Health consequences of adverse childhood experiences: A systematic review. Journal of the American Association of Nurse Practitioners, 27(8), 457-465. http://doi.org/10.1002/2327-6924.12215

Kearns, M. C., Edwards, K. M., Calhoun, K. S., \& Gidycz, C. A. (2010). Disclosure of sexual victimization: The effects of Pennebaker's emotional disclosure paradigm on physical and psychological distress. Journal of Trauma and Dissociation, 11(2), 193-209. http://doi.org/10.1080/15299730903502979

Khanna, N., \& Madoori, S. (2013). Untangling the Intersection of HIV and Trauma: Why It Matters and What We Can Do. Retrieved from http://www.thebody.com/content/73194/untangling-the-intersection-of-hiv-andtrauma-why-.html

Kilroy, A., Garner, C., Parkinson, C., Kagan, C., \& Senior, P. (2007). Towards Transformation: Exploring the impact of culture, creativity and the arts on health and wellbeing.

Knight, E. K. (2014). Shifting Public Health Practice to Advance Health Equity. Journal of Public Health Management and Practice, 20(2), 188-196.

http://doi.org/10.1097/PHH.0b013e31829959fb 
Lakoff, G. (2008). The Political Mind: A Cognitive Scientist's Guide to Your Brain and Its Politics. New York: Penguin Books.

Leavy, P. (2015). Method Meets Art: Arts-Based Research Practice (Second Edi). New York: The Guilford Press.

Leitch, L. (2017). Action steps using ACEs and trauma-informed care: a resilience model. ,. Health \& Justice, 5(5). http://doi.org/http://doi.org/10.1186/s40352-017$0050-5$

Ligenza, L. (2011). Promises to Keep: Interview with Ann Jennings. National Council Magazine, 2011(2), 34-36.

Link, B. G., \& Phelan, J. C. (2006). Stigma and its public health implications. Lancet, 367(9509), 528-529. http://doi.org/10.1016/S0140-6736(06)68184-1

Lister, C., Payne, H., Hanson, C. L., Barnes, M. D., Davis, S. F., \& Manwaring, T. (2017). The Public Health Innovation Model: Merging Private Sector Processes with Public Health Strengths. Frontiers in Public Health, 5, 192.

http://doi.org/10.3389/fpubh.2017.00192

Little, D. (2008). What is hermeneutic explanation? Dearborn, MI. Retrieved from http://www-personal.umd.umich.edu/ delittle/Encyclopedia entries/hermeneutic explanation.htm

Longino, C. F., \& Murphy, J. W. (1995). The old age challenge to the biomedical model : paradigm strain and health policy. Baywood Pub. Retrieved from https://books.google.com/books/about/The_Old_

Lynn-Whaley, J., \& Sugarmann, J. (2017). The relationship between community violence and trauma. Los Angeles. Retrieved from www.vpc.org. 
MacDonald, C. (2012). Understanding Participatory Action Research: A qualitative research methodology option. Canadian Journal of Action Research, 13(2), 34-50. Retrieved from http://ezproxy.lib.ucalgary.ca/login?url=http://search.ebscohost.com/login.aspx?dire $\mathrm{ct}=$ true $\& \mathrm{db}=\mathrm{ehh} \& \mathrm{AN}=89233336 \&$ site=ehost-live

Mackrain, M. (2013). Devereux Adult Resilience Survey (DARS). Retrieved from WWw.centerforresilientchildren.org.

Magruder, K. M., Kassam-Adams, N., Thoresen, S., \& Olff, M. (2016). Prevention and public health approaches to trauma and traumatic stress: A rationale and a call to action. European Journal of Psychotraumatology, 7, 1-10. http://doi.org/10.3402/ejpt.v7.29715

Magruder, K. M., McLaughlin, K. A., \& Elmore Borbon, D. L. (2017). Trauma is a public health issue. European Journal of Psychotraumatology, 8(1), 1375338. http://doi.org/10.1080/20008198.2017.1375338

Markusen, A., \& Gadwa, A. (2010). Creative Placemaking. Washington, D.C.

Mayfield-Johnson, S., \& Butler, J. (2017). Moving from Pictures to Social Action: An Introduction to Photovoice as a Participatory Action Tool. New Directions for Adult and Continuing Education, 2017(154), 49-59. http://doi.org/10.1002/ace.20230

Minkler, M. (2000). Using Participatory Action Research to build Healthy Communities. Public Health Reports, 115(2-3), 191-197. http://doi.org/10.1093/phr/115.2.191

Morgan, M. A., \& Lifshay, J. (2006). Community Engagement in Public Health. Contra Costa Health Services Public Health Division, 1-8. Retrieved from https://cchealth.org/public-health/pdf/community_engagement_in_ph.pdf 
Muirhead, A., \& De Leeuw, S. (2012). Emerging Priorities: Art and wellness: the importance of art for Aboriginal peoples' health and healing, 1-8. Retrieved from https://artshealthnetwork.ca/ahnc/art_wellness_en_web.pdf

Napier, A. D., Ancarno, C., Butler, B., Calabrese, J., Chater, A., Chatterjee, H., ... Woolf, K. (2014). Culture and health. The Lancet, 384(9954), 1607-1639. http://doi.org/10.1016/S0140-6736(14)61603-2

National Child Traumatic Stress Network (NCTSN). (2018). Complex Trauma. Retrieved from https://www.nctsn.org/what-is-child-trauma/trauma-types/complex-trauma National Coalition against Domestic Violence (NCADV). (2018). National Statistics. Retrieved from https://ncadv.org/statistics

Newman, T., Curtis, K., \& Stephens, J. (2003). Do community-based arts projects result in social gains? A review of the literature. Community Development Journal, 38(4), 310-322. http://doi.org/10.1093/cdj/38.4.310

Nguyen, A. (2015). Theoretical Approaches: Social Work Systems Theory. Retrieved from https://socialworklicensemap.com/theoretical-approaches-social-worksystems-theory/

Nicholls, S. (2009). Beyond expressive writing: Evolving models of developmental creative writing. Journal of Health Psychology, 14(2), 171-180. http://doi.org/10.1177/1359105308100201

Nivens, S. (2015). A Guide to GPRA Data Collection Using Trauma-Informed Interviewing Skills. Retrieved from https://www.integration.samhsa.gov/aboutus/Trauma-InformedInterviewingManual-508.pdf 
Nixon, L., Somji, A., Mejia, P., Dorfman, L., \& Quintero, F. (2015). Talking About Trauma.

Office of Juvenile Justice and Delinquency Prevention (OJJDP). (2018). Specialized Responses for Girls in the Juvenile Justice System. Washington, D.C. Retrieved from www.ojjdp.gov

Okagaki, L. H., \& Dean, R. A. (2016). The influence of funding sources on the scientific method. Molecular Plant Pathology, 17(5), 651-3. http://doi.org/10.1111/mpp.12380

O’Mara-Eves, A., Brunton, G., Oliver, S., Kavanagh, J., Jamal, F., \& Thomas, J. (2015). The effectiveness of community engagement in public health interventions for disadvantaged groups: a meta-analysis. BMC Public Health, 15(1), 129. http://doi.org/10.1186/s12889-015-1352-y

Ombler, J., \& Donovan, S. (2017). What does art have to do with public health, and how can they work together? - Public Health Expert, University of Otago, New Zealand. Retrieved from https://blogs.otago.ac.nz/pubhealthexpert/2017/04/10/what-does-arthave-to-do-with-public-health-and-how-can-they-work-together/

Oral, R., Ramirez, M., Coohey, C., Nakada, S., Walz, A., Kuntz, A., ... Peek-Asa, C. (2016). Adverse childhood experiences and trauma informed care: the future of health care. Pediatric Research, 79(1-2), 227-233. http://doi.org/10.1038/pr.2015.197

Pain, H. (2012). A Literature Review to Evaluate the Choice and Use of Visual Methods. International Journal of Qualitative Methods, 11(4), 303-319. http://doi.org/10.1177/160940691201100401 
Pan American Health Organization (PAHO). (2011). Population and individual approaches to the prevention and management of diabetes and obesity. Washington, D.C. Retrieved from https://www.paho.org/hq/dmdocuments/2012/DMPLANENGLISH.pdf

Paris, D. (2012). Culturally Sustaining Pedagogy: A Needed Change in Stance, Terminology, and Practice. Educational Researcher, 41(3), 93-97. http://doi.org/10.3102/0013189X12441244

Parkinson, C., \& White, M. (2013). Inequalities, the arts and public health: Towards an international conversation. Arts and Health, 5(3), 177-189. http://doi.org/10.1080/17533015.2013.826260

PEACC. (2018). About Us - UofL PEACC Center. Retrieved from https://louisville.edu/peacc/about-us

Pennebaker, J. (2006). Opening Up: The Healing Power of Expressing Emotions. New York: Guilford Press.

Pennebaker, J. W., \& Seagal, J. D. (1999). Forming a Story: The Health Benefits of Narrative. J Clin Psychol, 55(10), 1243-1254. http://doi.org/10.1002/(SICI)10974679(199910)55:10\&lt;1243::AID-JCLP6\&gt;3.0.CO;2-N

Pennebaker, J. W. (2000). Telling Stories: The Health Benefits of Narrative. Literature and Medicine, 19(1), 3-18. http://doi.org/10.1353/lm.2000.0011

Peters, J. (2016). Healthy families: from ACEs to trauma informed care to resilience and wellbeing: examples of policies and activities across IIMHL \& IIDL countries. Retrieved from http://www.iimhl.com/files/docs/Make_It_So/20161206.pdf 
Pinderhughes, H., Davis, R. A., \& Williams, M. (2015). Adverse Community Experiences and Resilience. Oakland, CA.

Putland, C. (2008). Lost in translation: The question of evidence linking communitybased arts and health promotion. Journal of Health Psychology. http://doi.org/10.1177/1359105307086706

Ramírez-Esparza, N., \& Pennebaker, J. W. (2006). Do good stories produce good health?: Exploring words, language, and culture. Narrative Inquiry, 16(1), 211-219. http://doi.org/10.1075/ni.16.1.26ram

Randall, M. (2010). Sexual Assault Law, Credibility, and "Ideal Victims": Consent, Resistance, and Victim Blaming. Canadian Journal of Women and the Law, 22(2), 397-433. http://doi.org/10.3138/cjwl.22.2.397

Rigg, K. K., Cook, H. H., \& Murphy, J. W. (2014). Expanding the scope and relevance of health interventions: Moving beyond clinical trials and behavior change models. International Journal of Qualitative Studies on Health and Well-Being, 9(1), 1-8. http://doi.org/10.3402/qhw.v9.24743

Rollins, J., Crane, L., Koff, R., Bieber, L., Winter, F., \& Sansom, I. (2011). Arts Organizations \& Public Health. Washington, D.C.

Ropeik, D. (2004). The consequences of fear. EMBO Reports, 5 Spec No(Suppl 1), S5660. http://doi.org/10.1038/sj.embor.7400228

Rosenberg, J. (2017). The Effects of Chronic Fear on a Person's Health. In Neuroscience Education Institute (NEI) 2017 Congress. AJMC Managed Markets Network. Retrieved from https://www.ajmc.com/conferences/nei-2017/the-effects-of-chronicfear-on-a-persons-health 
Saar, M. S., Epstein, R., Rosenthal, L., \& Vafa, Y. (2015). The Sexual Abuse to Prison Pipeline: The Girls'Story. Rights4Girls. Retrieved from www.law.georgetown.edu/go/poverty

Saeri, A. K., Cruwys, T., Barlow, F. K., Stronge, S., \& Sibley, C. G. (2018). Social connectedness improves public mental health: Investigating bidirectional relationships in the New Zealand attitudes and values survey. Australian and New Zealand Journal of Psychiatry. http://doi.org/10.1177/0004867417723990

SAMHSA. (2014). SAMHSA's Concept of Trauma and Guidance for a TraumaInformed Approach. HHS Publication, (July), 27.

SAMHSA. (2016). Cultural Competence. Retrieved from https://www.samhsa.gov/capt/applying-strategic-prevention/cultural-competence SAMHSA. (2018). Adverse Childhood Experiences. Retrieved from https://www.samhsa.gov/capt/practicing-effective-prevention/prevention-behavioralhealth/adverse-childhood-experiences

SAMHSA. (2018). Trauma and Violence. Retrieved from https://www.samhsa.gov/trauma-violence

Sanon, M. A., Evans-Agnew, R. A., \& Boutain, D. M. (2014). An exploration of social justice intent in photovoice research studies from 2008 to 2013. Nursing Inquiry, 21(3), 212-226. http://doi.org/10.1111/nin.12064

Schwarzer, R., \& Warner, L. M. (2013). Perceived self-efficacy and its relationship to resilience. In The Springer series on human exceptionality: Resilience in children, adolescents, and adults: Translating research into practice.

http://doi.org/10.1007/978-1-4614-4939-3 
Seppala, E. (2014). Connectedness and Health: The Science of Social Connection.

Retrieved from http://ccare.stanford.edu/uncategorized/connectedness-health-thescience-of-social-connection-infographic/

Shankar, A. (2016). Critique of the Photovoice Method, 32(2), 157-166.

http://doi.org/10.1111/var.12107.158

Shepherd, S. M., Delgado, R. H., Sherwood, J., \& Paradies, Y. (2018). The impact of indigenous cultural identity and cultural engagement on violent offending. $B M C$ Public Health, 18, 50.

Sherman, F. (2005). Detention reform and girls. Baltimore, MD.

Sherman, F. T., Balck, A., Bangura, R., Canfield, A., Chambers, B., Domingo-Salu, J., ... Shoenberg, D. (2015). Gender injustice: System-level juvenile justice reforms for girls. Portland, OR. Retrieved from www.nationalcrittenton.org/gender-injustice

Shotter, J. (2014). Agential realism, social constructionism, and our living relations to our surroundings: Sensing similarities rather than seeing patterns. Theory \& Psychology, 24(3), 305-325. http://doi.org/10.1177/0959354313514144

Simmonds, S., Roux, C., \& Avest, I. ter. (2015). Blurring the boundaries between photovoice and narrative methods: Gender-based research through narrativephotovoice. International Journal of Qualitative Methods, 14(3), 33-49. http://doi.org/10.1177/160940691501400303

Singer, M., Dressler, W., George, S., Baquet, C. R., Bell, R. A., Burhansstipanov, L., ... Williams, D. (2016). Culture: The missing link in health research. Social Science and Medicine, 170, 237-246. http://doi.org/10.1016/j.socscimed.2016.07.015 
Snyder, C. R. (2002). Hope Theory: Rainbows in the Mind. Psychological Inquiry. Taylor \& Francis, Ltd. http://doi.org/10.2307/1448867

Stanton, C. R. (2014). Crossing Methodological Borders: Decolonizing CommunityBased Participatory Research. Qualitative Inquiry, 20(5), 573-583. http://doi.org/10.1177/1077800413505541

Stevens, A. (2018). Sarabande Anthology Features Young Louisville Poets' Writings On Violence. Retrieved from https://wfpl.org/upcoming-sarabande-anthology-featuresyoung-poets-on-violence/

Strack, R. W., Lovelace, K. A., Jordan, T. D., \& Holmes, A. P. (2010). Framing Photovoice Using a Social-Ecological Logic Model as a Guide. Health Promotion Practice, 11(5), 629-636. http://doi.org/10.1177/1524839909355519

Strack, R. W., Magill, C., \& McDonagh, K. (2004). Engaging youth through photovoice. Health Promotion Practice, 5(1), 49-58. http://doi.org/10.1177/1524839903258015 Stuckey, H., \& Nobel, J. (2010). The Connection Between Art, Healing, and Public Health: A Review of Current Literature. American Journal of Public Health, 100(2), 253-263.

Swanbon, T., Boyce, L., \& Greenberg, M. A. (2008). Expressive writing reduces avoidance and somatic complaints in a community sample with constraints on expression. British Journal of Health Psychology, 13(1), 53-56.

http://doi.org/10.1348/135910707X251180

Tariq, S., \& Woodman, J. (2013). Using mixed methods in health research. JRSM Short Reports, 4(6), 2042533313479197. http://doi.org/10.1177/2042533313479197 
Tarr, J., Gonzalez-Polledo, E., \& Cornish, F. (2018). On liveness: using arts workshops as a research method. Qualitative Research, 18(1), 36-52. http://doi.org/10.1177/1468794117694219

Teti, M., Conserve, D., Zhang, N., \& Gerkovich, M. (2016). Another way to talk: Exploring photovoice as a strategy to support safe disclosure among men and women with HIV. AIDS Education and Prevention, 28(1), 43-58. http://doi.org/10.1521/aeap.2016.28.1.43

Travagin, G., Margola, D., \& Revenson, T. A. (2015). How effective are expressive writing interventions for adolescents? A meta-analytic review. Clinical Psychology Review, 36, 42-55. http://doi.org/10.1016/j.cpr.2015.01.003

Trickett, E. J., Beehler, S., Deutsch, C., Green, L. W., Hawe, P., McLeroy, K., ... Trimble, J. E. (2011). Advancing the science of community-level interventions. American Journal of Public Health, 101(8), 1410-1419. http://doi.org/10.2105/AJPH.2010.300113

Trujillo, M. D., \& Plough, A. (2016). Building a culture of health: A new framework and measures for health and health care in America. Social Science and Medicine, 165, 206-213. http://doi.org/10.1016/j.socscimed.2016.06.043

Tsiris, G., Tasker, M., Lawson, V., Prince, G., Dives, T., Sands, M., \& Ridley, A. (2011). Music and Arts in Health Promotion and Death Education: The St Christopher's Schools Project. Music and Arts in Action, 3(2), 95-119. Retrieved from http://bergh.fm/musicandartsinaction.net/index.php/maia/article/view/stchristopherss choolsproject 
Umberson, D., \& Montez, J. K. (2010). Social Relationships and Health: A Flashpoint for Health Policy. Journal of Health and Social Behavior, (51(Suppl)), S54-S66. http://doi.org/10.1177/0022146510383501

Unger, J. (2011). Cultural Identity and Public Health. In Handbook of Identity Theory and Research (pp. 811-825). New York, NY: Springer. http://doi.org/doi:10.1007/978-1-4419-7988-9_34

Van der Kolk, B. A. (n.d.). The body keeps the score : brain, mind, and body in the healing of trauma.

Van der Riet, M. (2008). Participatory Research and the Philosophy of Social Science. Qualitative Inquiry, 14(4), 546-565. http://doi.org/10.1177/1077800408314350

Veysey, B. M. (2003). Adolescent Girls with Mental Health Disorders Involved with the Juvenile Justice System. Delmar, NY. Retrieved from www.ncmhjj.com

Viar, S. (2017). CU Scholar Gender-Specific Rehabilitation for Justice Involved Youth: Is this Treatment Warranted? Boulder. Retrieved from https://scholar.colorado.edu/honr_theseshttps://scholar.colorado.edu/honr_theses/14 65

Wang, C. C. (1999). Photovoice-A Participatory Action Research Strategy Applied to Women's Health. Journal of Women's Health, 8(2), 185-192.

Wang, C., \& Burris, M. A. (1997). Photovoice: Concept, Methodology, and Use for Participatory Needs Assessment. Health Education \& Behavior, 24(3), 369-387. http://doi.org/10.1177/109019819702400309

Wang, C., Yi, W. K., Tao, Z. W., \& Carovano, K. (1998). Photovoice as a participatory health promotion strategy. Health Promotion International, 13(1), 75-86. 
Wang, C., Morrel-Samuels, S., Mutchison, P., Bell, L., \& Pestronk, R. (2004). Flint Photovoice- Community Building Among Youth, Adults and Policymakers. American Journal of Public Health, 94(6), 911-. http://doi.org/10.2105/AJPH.94.6.911

World Health Organization (WHO) Regional Office for Europe. (2011). Evidence for gender responsive actions to prevent violence. Copenhagen. Retrieved from http://www.euteach.com/euteach_home/euteach_network.

Wright, J., Williams, R., \& Wilkinson, J. R. (1998). Development and importance of health needs assessment. BMJ (Clinical Research Ed.), 316(7140), 1310-3. Retrieved from http://www.ncbi.nlm.nih.gov/pubmed/9554906

Yonas, M. A., O’campo, P., Burke, J. G., Peak, G., \& Gielen, A. C. (2005). Urban Youth Violence: Do Definitions and Reasons for Violence Vary by Gender? Journal of Urban Health: Bulletin of the New York Academy of Medicine, 82(4). http://doi.org/10.1093/jurban/jti077

Zahn, M. A., Day, J. C., Mihalic, S. F., \& Tichavsky, L. (2009). Determining What Works for Girls in the Juvenile Justice System. Crime \& Delinquency, 55(2), 266293.

Zoughbie, D. (2013). Good Health Can Be Contagious. Retrieved from https://www.huffingtonpost.com/daniel-zoughbie/health-initiative_b_2821964.html 


\section{CURRICULUM VITA}

NAME: $\quad$ Tasha L. Golden

ADDRESS: Department of Health Promotion and Behavioral Sciences 485 E. Gray St.

Louisville, KY 40202

EDUCATION: $\quad$ Bachelor of Music, Music Composition

Cincinnati Christian University

Master of Arts, English

Miami University

\section{AWARDS:}

- Doctoral Dissertation Completion Award, 2019

- Health and Social Justice Scholarship, 2016-2019

- Outstanding Graduate Student Award, 2018

- Carolyn Krause Maddox Prize for Research, 2016

- Randy Martin Prize for Research, 2015

\section{PUBLICATIONS:}

Wendel, M., Jackson, T., Ingram, M., Golden, T., Castle, B.F., Ali, N., \& Combs, R. (2019). "Yet We Live, Strive, and Succeed:" Using photovoice to understand residents' experience of justice, safety, hope, and racial equity, Collaborations: A Journal of Community-Based Research and Practice.

Golden, T. and Hand, J. Arts, Culture and Community Mental Health, Community Development Investment Review (13)1.

Golden, T. (2018). Writing with incarcerated teen women: Trauma-informed pedagogy, health and gender equity. In Lockard \& Rankins-Robertson (Eds.), Prison Pedagogies: Learning and Teaching with Imprisoned Writers. Syracuse, NY: Syracuse University Press.

Golden, T. (2017). Subalternity in juvenile justice: Gendered oppression and the rhetoric of reform, Reflections Journal of Public Rhetoric (17), 156-188.

Golden, T. (2017). Pop Heresy: Songwriting at the edge of the speakable. In Erickson \& Schweizer (Eds.), Reading Heresy: Religion and Dissent in Literature and Art. De Gruyter Press. 
Golden, T. (2013). "Hope" in "The People, Yes": Carl Sandburg and the role of hope in contemporary America. Ethos Journal.

\section{SELECT INVITED PRESENTATIONS:}

- "Thinking Creatively to Advance a Culture of Health" National Academy of Medicine, Washington, D.C., March 2019

- "Public Health Policies and Priorities: Potential Roles for the Arts" "Creating Health Communities" National Initiative, Cincinnati, OH, June 2018

- "How Art \& Performance Can Improve Girls' Health, Amplify their Voices, and Support PACE Advocacy Efforts" PACE Center for Girls 'All About Girls Summit,' Orlando, FL, March 2018

- "The Arts as a Tool for Change: Girls, Voice, Advocacy" National Crittenton Foundation, "Girls @ the Margins" Webinar, September 2017

- $\quad$ "The Art of the Unspeakable: How Public Creativity Challenges Stigma and Silence"

Plenary Talk, Southern Humanities Council National Conference, Louisville, KY, January 2016

- "Art and the Public Humanities"

University of Notre Dame MFA Teaching Practicum Guest Lectures, 2014-2015

\section{SELECT NATIONAL CONFERENCE PRESENTATIONS:}

- "Creating Public Health: Art's Role in Culturally Responsive, Trauma- Informed Public Health Practice" a2ru National Conference, Athens, GA, November 2018

- "The Power of Voice: How Arts Programming Can Build Resilience, Improve Health, and Challenge Gender Inequity in Juvenile Justice" Association for Justice-Involved Females and Organizations, Santa Clara, CA December 2017

- "Where Art Meets Public Health: How Performance, Writing, and Project HEAL Are Advancing Health Equity in Louisville" Smithsonian Festival of Innovation, Smithsonian Museum of American History, Washington, D.C., October 2017

- "Performance at the Edge of the Speakable" International Health and Humanities Conference, Cleveland, OH, March 2016

- "Gendered Oppression: Juvenile Justice and the Rhetoric of Reform" National Cultural Studies Association Conference, Riverside, CA, May 2015

\section{PROFESSIONAL AFFILIATIONS:}

- Association for Women in Science (AWS)

- American Psycological Association (APA), Division 10

- Association for Justice-Involved Females and Organizations (AJJFO)

- Kentucky Academy of Science (KAS)

- Kentucky Public Health Association (KPHA)

- The American Society of Composers, Authors, and Publishers (ASCAP) 